SERVIÇO DE PÓS-GRADUAÇÃO DO ICMC-USP Data de Depósito: 08.03.2005

Assinatura: Ana Pauda lampaio ruelsn.

\title{
Dicotomias em equações diferenciais impulsivas
}

\author{
Angela Leite Moreno
}

Orientadora: Profa. Dra. Márcia Cristina Anderson Braz Federson

Dissertação apresentada ao Instituto de Ciências Matemáticas e de Computação - ICMC-USP, como parte dos requisitos para obtenção do título de Mestre em Matemática.

\section{USP - São Carlos}

Março/2005 
Aluno: Angela Leite Moreno

A Comissã̃o Julgadora:

Profa. Dra. Márcia Cristina Anderson Braz Federson Mária Fedeece.

Profa. Dra. Maria do Carmo Carbinatto

Mani do Camo Canhirato

Prof. Dr. Aloisio José Freiria Neves

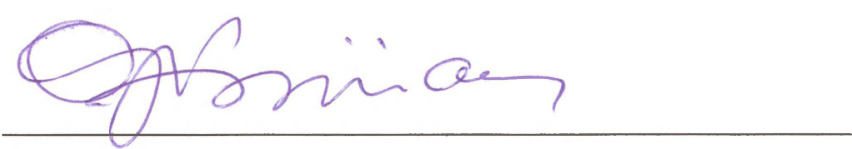


Ao meu marido

Roberto

e à minha filha Carolina. 


\section{Agradecimentos}

Há muitos a quem agradecer, pois nenhuma pesquisa científica é solitária. Ao longo caminho, fui orientada, inspirada e apoiada por todas as pessoas que em mim confiaram.

Agradaço, primeiramente, à Deus, por estar sempre presente em meu coração guiando-me pelos caminhos da vida.

Em particular, agradeço profundamente ao meu marido, Roberto, por seu amor, sua amizade e compreensão e à minha filha, Carolina, pela alegria que traz à minha vida.

Aos meus pais, Sr. Edivá e Sra. Conceição, por terem apoiado meus passos na infância, os conselhos na adolescência e ensinamentos para toda vida; e às minhas irmãs, Rosinéia e Rosangela, por acreditarem em mim.

Aos amigos de sempre, Alcindo, Carlos, Christiano, Danielli, Eliza, Marleide, Nair, Pandu e Solange, pela grande amizade, pela dedicação e por todo apoio que deram durante estes anos.

À minha orientadora professora Dra. Márcia Cristina Anderson Braz Federson, que com perícia me ajudou a vencer as dificuldades e me orientou com competência e dedicação.

Não tenho palavras para expressar também minha gratidão a:

Aos meus amigos de graduação do curso de Licenciatura Plena em Matemática da Universidade Estadual Paulista - UNESP, campus de Presidente Prudente, SP

Aos meus amigos do primeiro ano de gradução do curso de Licenciatura Plena em Matemática da Universidade Federal do Mato Grosso - UFMS, campus de Três Lagoas, MS.

Aos meus amigos de minha turma de mestrado (Aldicio, Andréa, Daniela, Everaldo, Fábio, 
Márcio Alexandre, Márcio, Nivaldo, Rafael, Rodrigo, Thiago e Vanda), pelo companheirismo, amizade e por tudo que passamos juntos no início de nossa caminhada.

Aos professores da FCT, em especial aos professores Dr. José Carlos Rodrigues e Dr. José Roberto Nogueira, pelo aconselhamento e incentivo a continuar os estudos.

Aos professores da UFMS de Três Lagoas, especialmente ao professor Dr. Antônio Carlos Tamarozzi, pelo incentivo no início de minha caminhada.

Aos professores e funcionários da USP, meu muito obrigado por tudo!

Finalmente, ao $\mathrm{CNPq}$, pelo apoio financeiro para a realização deste trabalho. 


\section{Resumo}

Neste trabalho, tratamos da teoria fundamental de dicotomias para certa equação diferencial linear com impulsos a tempo pré-fixado. Consideramos condições necessárias e suficientes para a existência de dicotomia exponencial e dicotomia ordinária para esta equação e apresentamos algumas consequiências destes fatos. Escrevemos abreviadamente EDI para significar equação diferencial impulsiva.

Alguns dos resultados interessantes contidos neste texto estão descritos a seguir. Apresentamos algumas relações entre crescimento limitado e dicotomia exponencial para a EDI em estudo. Apresentamos, também, condições para a equivalência entre a existência de dicotomia exponencial para a EDI que tratamos e a admissibilidade de certos pares de funções para uma perturbação desta EDI. Em particular, se nossa EDI tiver uma dicotomia exponencial, então, dada certa perturbação limitada, obtemos uma EDI não-homogênea que admitirá solução também limitada e vale a recíproca. Nós contribuímos com este resultado provando o fato de que o espaço das funções limitadas pode ser substituído pelo espaço das funções limitadas com limite no infinito. Assim, se a EDI tiver uma dicotomia exponencial, então dada uma perturbação limitada com limite no infinito, a EDI perturbada admitirá uma solução também limitada e com limite no infinito e a recíproca é verdadeira.

Outro resultado importante diz que se a EDI estudada for quase periódica e tiver uma dicotomia exponencial sobre $\mathbb{R}_{+}$, então ela terá uma dicotomia exponencial sobre toda a reta. E a nossa contribuição aqui se deu através de um resultado mais geral que diz que se a EDI tiver uma dicotomia sobre um intervalo finito de comprimento suficientemente grande, então ela terá uma dicotomia sobre toda a reta. 


\begin{abstract}
In this work we deal with the fundamental theory of dichotomies for a linear differential equation with pre-assigned moments of impulse effects. We consider necessary and sufficient conditions for the existence of each of two types of dichotomies for this equation: ordinary and exponential dichotomies, and we present some consequences of these facts. We write IDE for impulsive differential equation.

Some interesting results are mentioned below. We present some relations between bounded growth and the existence of exponential dichotomy for the IDE in question. We also present the equivalence between the existence of an exponential dichotomy for our IDE and the admissibility of certain pairs of function for the IDE with a perturbation. In particular, if our IDE has an exponential dichotomy, then given certain bounded perturbation, we obtain a non-homogeneous IDE which admits a bounded solution and the converse holds. We contribute to this result showing that the space of bounded functions can be replaced by the space of bounded function with limit at infinity. This means that if our IDE has an exponential dichotomy, then for any bounded perturbation with limit at infinity, the perturbed IDE admits a bounded solution with limit at infinity and we also have a converse.

Another interesting result says that if the IDE we study is almost periodic and has an exponential dichotomy on $\mathbb{R}_{+}$then it also has an exponential dichotomy on $\mathbb{R}$. We generalize this results proving that if our IDE has an exponential dichotomy on a finite interval of sufficiently large length, then the IDE also has an exponential dichotomy on $\mathbb{R}$.
\end{abstract}




\section{Sumário}

1 Teoria Fundamental 5

1.1 Espaços de Banach $\ldots \ldots \ldots \ldots \ldots \ldots$

1.1.1 Espaços Normados e Espaços de Banach . . . . . . . . . . . . 5

1.1 .2 Operadores Lineares $\ldots \ldots \ldots \ldots \ldots$

1.1.3 Soma Direta de Subespaços e Projeções . . . . . . . . . . . . . . . 9

1.1.4 Complexificação de um Espaço de Banach . . . . . . . . . . . . . . 10

1.2 Operadores Lineares Limitados . . . . . . . . . . . . . . . . . 11

1.2.1 Espectro e Resolvente . . . . . . . . . . . . . . . . . . 11

$1.2 .2 \quad$ A Integral de Riesz . . . . . . . . . . . . . . . . . 12

2 Equações Diferenciais Impulsivas 15

2.1 Definições Básicas . . . . . . . . . . . . . . . . . . . . . . 15 
2.2 Existência e Unicidade de Soluções . . . . . . . . . . . . . . . . . . . . 17

2.3 Desigualdades Integrais . . . . . . . . . . . . . . . . . . . . . 20

3 Dicotomias para Equações Diferenciais Impulsivas $\quad 28$

3.1 Definições e Propriedades . . . . . . . . . . . . . . . . . . 28

3.2 Redutibilidade . . . . . . . . . . . . . . . . . . . 44

4 Crescimento Limitado e Dicotomias $\quad 50$

4.1 Definições e Propriedades . . . . . . . . . . . . . . . . . . 50

4.2 Relações entre Crescimento Limitado e Dicotomia Exponencial . . . . . . . . . 61

5 Dicotomias e Admissibilidade $\quad 70$

5.1 Dicotomia Ordinária e Admissibilidade . . . . . . . . . . . . . . . 72

5.2 Dicotomia Exponencial e Admissiblidade . . . . . . . . . . . . . . . 79

5.2.1 Admissibilidade de Funções com Limite no Infinito . . . . . . . . . . . . 87

6 Dicotomia Exponencial e Funções Quase Periódicas 93

$\begin{array}{lr}\text { Apêndice 1: Um Teorema do Tipo Áscoli-Arzelá } & 117\end{array}$

$\begin{array}{ll}\text { Referências Bibliográficas } & 120\end{array}$ 


\section{Introdução}

O estudo das equações diferenciais impulsivas (EDIs) é muito importante pois elas modelam, de forma mais realista, vários problemas que surgem naturalmente em diversas áreas das ciências e tecnologia como, por exemplo, em teoria de controle, engenharia (robótica industrial), física (mecânica), ciências médicas e biológicas (farmacocinética, dinâmica populacional), economia entre muitas outras. As EDIs descrevem a evolução de sistemas onde o desenvolvimento contínuo de um processo sofre variações de estado de curta duração que podem ser consideradas instantâneas. Esse fenômeno é chamado impulso ou ação impulsiva e corresponde às descontinuidades de primeira espécie das soluções.

No presente trabalho, consideramos o caso de EDIs com impulsos pré-estabelecidos, ou seja, os impulsos são conhecidos de antemão, e apresentamos um estudo sobre dicotomias para estas EDIs e suas implicações no comportamento das soluções. O texto base para este trabalho foi [1]. Aqui, além de discutirmos vários resultados, nós também modificamos algumas demonstrações e incluímos outras. Também agregamos alguns resultados de artigos ao texto desta dissertação e contribuímos com alguns resultados novos.

Organizamos os capítulos desta dissertação da forma seguinte. No Capítulo 1, apresentamos algumas propriedades básicas sobre espaços de Banach que serão utilizadas no decorrer do texto. A referência fundamental para este capítulo é [7]. Vamos omitir as demonstrações pois estas são bem conhecidas e podem ser encontradas em [7].

No Capítulo 2, descrevemos uma EDI usual e definimos o que é uma solução para esta EDI. 
Apresentamos teoremas sobre existência e unicidade de soluções e estabelecemos algumas propriedades da matriz de Cauchy para EDIs. Damos condições para valerem certas desigualdades integrais para funções contínuas por partes, que serão utilizadas posteriormente. As referências principais para este capítulo são [1], [4] e [8].

No Capítulo 3, consideramos a EDI linear que será nosso objeto de estudo e apresentamos as definições de dicotomia exponencial, dicotomia ordinária e dicotomia espectral bem como algumas de suas propriedades. Além disso, definimos equivalência cinemática e apresentamos propriedades fundamentais deste conceito para nossa EDI. Os resultados contidos aqui podem ser encontrados em [1], [4] e [6].

No Capítulo 4, apresentamos vários resultados que relacionam crescimento limitado e existência de dicotomia exponencial para a EDI em questão. Primeiramente, damos condições necessárias e suficientes para que a EDI tenha crescimento limitado. Depois, relacionamos a existência de dicotomia exponencial e crescimento limitado de tal EDI. As referências para este capítulo são [1], [2] e [6].

No Capítulo 5, apresentamos condições para a equivalência entre a admissibilidade de certos pares de funções para uma perturbação de nossa EDI perturbada e a existência de uma dicotomia exponencial para a EDI original. Tais resultados podem ser encontrados em [1] e [9].

Finalizamos este trabalho com o Capítulo 6. Apresentamos um resultado já conhecido que diz que se a EDI (3.1), (3.2) for quase-periódica e tiver uma dicotomia exponencial sobre $\mathbb{R}_{+}$, então ela terá uma dicotomia exponencial sobre $\mathbb{R}$. Em seguida, apresentamos um resultado novo que nos diz que se esta EDI tiver uma dicotomia sobre um intervalo finito de comprimento suficientemente grande, então ela terá uma dicotomia sobre toda a reta. 


\section{Notação Básica}

Apresentamos, aqui, algumas notações que aparecem ao longo deste trabalho.

- $\mathbb{Z}$ representa o conjunto dos números inteiros;

- $\mathbb{Z}_{+}$representa o conjunto dos números inteiros não-negativos;

- $\mathbb{N}$ representa o conjunto dos números inteiros estritamente positivos;

- $\mathbb{R}$ representa o conjunto dos números reais;

- $\mathbb{R}_{+}$representa o conjunto dos números reais não-negativos;

- $\mathbb{R}_{-}$representa o conjunto dos números reais não-positivos;

- $\mathbb{C}$ representa o conjunto dos números complexos;

- $\operatorname{Re} z$ representa a parte real do número complexo $z$;

- Im $z$ representa a parte imaginária do número complexo z;

- $\mathbb{R}^{N}$ representa o espaço euclidiano $N$-dimensional, que denotamos por $X$;

- $\bar{A}$ representa o fecho de um conjunto $A \subset X$;

- $\partial A$ representa a fronteira de um conjunto $A \subset X$;

- $B(x, \varepsilon)=\{y \in \mathrm{X}: \rho(y, x)<\varepsilon\}$, onde $\rho(x, y)$ denota a distância entre $x$ e $y$;

- $\left(x_{1}, x_{2}\right) \in \mathbb{R}$ representa o produto interno usual entre $x_{1}$ e $x_{2}$;

- $\operatorname{Im} f$ representa o conjunto imagem de uma função $f$; 
- Ker $f$ representa o núcleo de uma função $f$.

Quando um conceito for introduzido pela primeira vez, as palavras que o definem estarão em negrito.

Indicaremos o final de uma demonstração com o símbolo $\square$. 


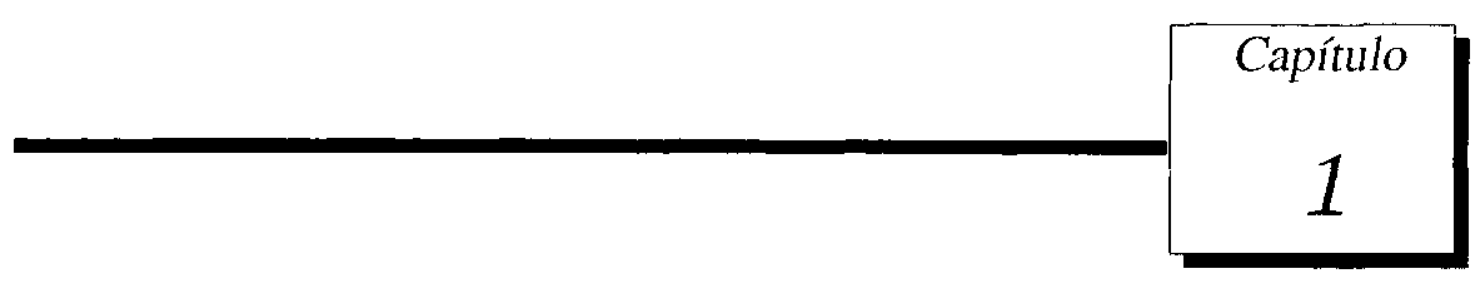

Teoria Fundamental

\subsection{Espaços de Banach}

Nesta seção, enunciaremos algumas proposições gerais sobre a geometria de espaços de Banach. Este conteúdo será importante para o desenvolvimento das próximas seções. A referência para este capítulo é [7], onde podem ser encontradas as provas para os resultados que apenas enunciamos aqui.

\subsubsection{Espaços Normados e Espaços de Banach}

Diremos que um conjunto $\mathscr{L}$ é um espaço normado real (complexo) se

(i) $\mathscr{L}$ for um espaço vetorial linear sobre o corpo dos números reais (complexos), e

(ii) para cada vetor $x \in \mathscr{L}$, cxistir um número não-negativo $\|x\|$, denominado norma de $x$, com as seguintes propriedades:

(a) $\left|\alpha x_{\ddagger}^{\prime}=\right| \alpha||^{\prime} x_{i}^{\prime} \mid$, para qualquer $x \in \mathscr{L}$ e qualquer número real (complexo) $\alpha$;

(b) ||$x+y \mid: \leq\}_{1}\left|x_{i}^{\prime}\right|+|| y||$, para quaisquer $x, y \in \mathscr{L}$ (desigualdade triangular);

(c) $\|x\|=0$ se, e somente se, $x=0$ 
A função $\rho(x, y)=\vdots \mid x-y \|$ em um espaço normado $\mathscr{L} \ni x, y$, define uma métrica neste espaço. Deste modo, $(\mathscr{L}, \rho)$ é um espaço métrico.

Uma seqüência $\left\{x_{n}\right\} \subset \mathscr{L}$ será uma seqüência de Cauchy, se $\lim _{n, m \rightarrow \infty}\left\|x_{n}-x_{m}\right\|_{\mid}^{\prime}=0$.

Ǔm espaço normado $\mathscr{L}$ será um espaço de Banach, se toda seqüência de Cauchy em $\mathscr{L}$ tiver um ponto limite $x \in \mathscr{L}$ para o qual $\lim _{n \rightarrow \infty}\left\|x_{n}-x\right\|=0$. Em outras palavras, um espaço de Banach $\mathscr{L}$ é complcto na métrica $\rho(x, y)=\left\|x-y^{\|}\right\|$.

Se outra norma $\|x\|_{2}$ for dada em um espaço normado $\mathscr{L}$, com norma $\|x\|_{1}$, então toda sequiência convergente com respeito à norma $\|x\|_{1}$ convergirá com respeito à norma $\|x\|_{2}$ se, e somente se, existir uma constante positiva $c_{1}$ tal que $\left\|x_{i_{2}} \leq c_{1}\right\| x \|_{1}$.

Duas normas $\|x\|_{1}$ e $\|x\|_{2}$ dadas em um espaço linear $\mathscr{L}$ serão topologicamente equivalentes, se a convergência em uma implicar a convergência na outra. Para isto é necessário e suficiente que existam constantes positivas $c_{1}$ e $c_{2}$ tais que

$$
c_{2} \leq \frac{\|x\|_{2}}{\|x\|_{1}} \leq c_{1}, \quad x \neq 0
$$

Diremos que dois espaços de Banach sāo isomorfos, se existir uma aplicação linear contínua injetora de um espaço sobre o outro. Esta aplicação será chamada de isomorfismo. Se o isomorfismo preservar a norma, então ele será chamado de isomorfismo isométrico.

\subsubsection{Operadores Lineares}

Sejam $\mathscr{B}_{1}, \mathscr{B}_{2} \mathrm{e} \mathscr{B} 3$ espaços de Banach. Úma aplicação $A: \mathscr{B}_{1} \rightarrow \mathscr{B}_{2}$ é um operador linear, se $A(\alpha x+\beta y)=\alpha A x+\beta A y$ para quaisquer números $\alpha, \beta$ e quaisquer $x, y \in \mathscr{B}_{1}$.

Um operador linear será contínuo, se ele for contínuo no ponto $x=0$.

A continuidade é equivalente à propriedade de limitação de um operador $A$, isto é, à finitude da quantidade

$$
\|A\|:=\sup \left\{\frac{\|A x\|_{2}}{\|x\|_{1}}: x \in \mathscr{B}_{1}, x \neq 0\right\}=\sup \left\{\|A x\|_{2}: x \in \mathscr{B}_{1},\|x\|_{1}=1\right\} .
$$


O conjunto dos opcradores lineares limitados $A: \mathscr{B}_{1} \rightarrow \mathscr{B}_{2}$ será denotado por $\left[\mathscr{B}_{1}, \mathscr{B}_{2}\right]$. Este conjunto é um espaço de Banach com norma (1.1) sob a definição natural das operações de soma de operadores e de multiplicação de um operador por um número:

$$
(A+B) x=A x+B x \quad \text { e } \quad(\alpha A) x=\alpha(A x) .
$$

Sejam $A: \mathscr{B}_{2} \rightarrow \mathscr{B}_{3}$ e $B: \mathscr{B}_{1} \rightarrow \mathscr{B}_{2}$ operadores lineares. A fórmula $C x=A(B x)$, para $x \in \mathscr{B}_{1}$, define um operador $C: \mathscr{B}_{1} \rightarrow \mathscr{B}_{3}$ que será chamado de produto dos operadores $A \subset B$. Neste caso, escrevemos $C=A B$. Se os operadores $A$ e $B$ deste produto forem limitados, isto é, se $A \in\left[\mathscr{B}_{2}, \mathscr{B}_{3}\right]$ e $B \in\left[\mathscr{B}_{1}, \mathscr{B}_{2}\right]$, então o operador $C$ será limitado, ou scja, $C \in\left[\mathscr{B}_{1}, \mathscr{R}_{3}\right]$. Além disso, teremos

$$
\|C\|=\left\|\left.A B\right|_{i} \leq\right\| A\|\||| B \|
$$

Um operador $B: \mathscr{B}_{2} \rightarrow \mathscr{B}_{1}$ será chamado de inverso do operador $A: \mathscr{B}_{1} \rightarrow \mathscr{B}_{2}$, e escrevemos $B=A^{-1}$, se $A B=I_{2}$ e $B A=I_{1}$, onde $I_{k}$ é o operador identidade em $\mathscr{B}_{k}$, ou seja $I_{k} x=x$, para qualquer $x \in \mathscr{B}_{k}, k=1,2$.

O conjunto de todos os operadores lineares limitados de um espaço de Banach $\mathscr{B}$ sobre ele mesmo será denotado por $[\mathscr{B}]$. Assim $[\mathscr{B}]:=[\mathscr{B}, \mathscr{B}]$.

Sob a operação de multiplicação definida acima (produto de operadores), [ $\mathscr{B}]$ será um espaço de Banach.

Por $A^{n}$ denotaremos a composição do operador $A \in[\mathscr{B}]$ com ele mesmo $n$ vezes.

Diremos que a função $(\cdot, \cdot): \mathscr{B} \times \mathscr{B} \rightarrow \mathbb{C}$ define um produto interno, se forem satisfeitas as seguintes propriedades:

(i) $(x, y)=\overline{(y, x)}$, para todo $x, y \in \mathscr{B}$;

(ii) $(\lambda x, y)-\lambda(x, y)$, para quaisquer $x, y \in \mathscr{B}$ e $\lambda \in \mathbb{C}$;

(iii) $\left(x_{1}+x_{2}, y\right)=\left(x_{1}, y\right)+\left(x_{2}, y\right)$, para quaisquer $x_{1}, x_{2}, y \in \mathscr{B}$

(iv) $(x, x)=0$ se, e somente se, $x=0$. 
Seja $\mathscr{B}$ um espaço de Banach com produto interno. Para que um operador linear $A: \mathscr{B} \rightarrow \mathscr{B}$ seja limitado, isto é, $A \in[\mathscr{B}]$, será necessário e suficiente que exista um operador linear $A^{*}: \mathscr{B} \rightarrow \mathscr{B}$ tal que $(A x, y)=\left(x, A^{*} y\right)$, para quaisquer $x, y \in \mathscr{B}$. Deste modo, se $A \in[\mathscr{B}]$, então $A^{*} \in[\mathscr{B}] \mathrm{e}$ $\left(A^{*}\right)^{*}=A$. O operador $A^{*}$ é chamado operador adjunto de $A$.

Um operador $A \in[\mathscr{B}]$ será um operador hermitiano se $A=A^{*}$.

O teorema a seguir é conhecido como Teorema de Banach. Este teorema nos diz que se tivermos um operador linear limitado injetor de $\mathscr{B}_{1}$ sobre $\mathscr{B}_{2}$, com $\mathscr{B}_{1}$ e $\mathscr{B}_{2}$ espaços de Banach, então seu inverso também será um operador linear limitado. Isto justifica a definição de espaços de Banach isomorfos dada na seção anterior.

Teorema 1.1 (Banach) Suponhamos que o operador $A \in\left[\mathscr{B}_{1}, \mathscr{B}_{2}\right]$ seja uma aplicação injetora de um espaço de Banach $\mathscr{B}_{1}$ sobre um espaço de Banach $\mathscr{B}_{2}$. Entāo $A^{-1} \in\left[\mathscr{B}_{2}, \mathscr{B}_{1}\right]$, isto é, o inverso de A também será um operador linear limitado.

Corolário 1.1 Suponhamos que, em um espaço linear $\mathscr{L}$, sejam dadas duas normas $\|x\|_{1}$ e $\|x\|_{2}$ que fazem de $\mathscr{L}$ espaços de Banach que denotamos por $\mathscr{B}_{1}$ e $\mathscr{B}_{2}$ respectivamente. Suponhamos, também, que $\|x\|_{2} \leq c_{1}\|x\|_{1}$, para $x \in \mathscr{L}$, onde $c_{1}$ é uma constante positiva. Então existirá uma constante positiva $c_{2}$ tal que $\|x\|_{1} \leq c_{2}\|x\|_{2} e$, conseqüentemente, as normas $\|x\|_{1} e\|x\|_{2}$ serão topologicamente equivalentes.

Teorema 1.2 (Princípio da Limitação Uniforme) Suponhamos que U seja um conjunto de operadores de $\left[\mathscr{B}_{1}, \mathscr{B}_{2}\right]$ tal que, para cada $x \in \mathscr{B}_{1}$, tenhamos

$$
\sup \{\|A x\|: A \in U\}<\infty
$$

Então U será limitado.

Em particular, segue deste teorema que se uma seqüência de operadores $\left\{A_{n}\right\}_{n \in \mathbb{N}} \subset\left[\mathscr{B}_{1}, \mathscr{B}_{2}\right]$ for tal que $A_{n} x$ converge, qualquer que seja $x \in \mathscr{B}_{1}$, então a igualdade $A x=\lim _{n \rightarrow \infty} A_{n} x$ definirá um operador linear contínuo $A \in\left[\mathscr{B}_{1}, \mathscr{B}_{2}\right]$. 
Enccrramos esta subseção com o Teorema da Aplicação Aberta e o Teorema do Gráfico fechado que são essenciais neste trabalho.

Teorema 1.3 (Teorema da Aplicação Aberta) Suponhamos que $A \in\left[\mathscr{B}_{1}, \mathscr{B}_{2}\right]$ seja sobrejetiva. Então o operador A será aberto.

Teorema 1.4 (Teorema do Gráfico Fechado) Seja $A \in\left[\mathscr{B}_{1}, \mathscr{B}_{2}\right]$ e suponhamos que seu gráfico seja fechado. Então o operador A será contínuo.

\subsubsection{Soma Direta de Subespaços e Projeções}

Um espaço de Banach $\mathscr{B}$ poderá ser decomposto como soma direta dos subespaços $\mathscr{B} \mathscr{B}_{1}$ e $\mathscr{B}{ }_{2}$ e, neste caso, escreveremos

$$
\mathscr{B}=\mathscr{B}_{1} \ominus \mathscr{B}_{2}
$$

se cada elemento $x \in \mathscr{B}$ admitir uma única representação na forma

$$
x=x_{1}+x_{2},
$$

onde $x_{1} \in \mathscr{B}_{1}$ e $x_{2} \in \mathscr{B}_{2}$.

Cada um dos subespaços $\mathscr{B}_{k}, k=1,2$, é dito complementar direto um do outro.

A decomposição (1.2) define dois operadores lineares $P_{k}: \mathscr{B} \rightarrow \mathscr{B}_{k}, k=1,2$, através das igualdades $P_{k} x=x_{k}$, onde $x_{1}$ e $x_{2}$ são as componentes de $x$ na decomposição (1.3). Os operadores $P_{1} \mathrm{e}$ $P_{2}$ tem as seguintes propriedades:

$$
P_{k}^{2}=P_{k} ; \quad P_{1}+P_{2}=I ; \quad P_{1} P_{2}=P_{2} P_{1}=0 ; \quad k=1,2 .
$$

Tais operadores também são contínuos, isto é, $P_{k} \in[\mathscr{B}], k=1,2$. Para provarmos este fato, seja

$$
\|x\|_{1}=\left\|x_{1} \mid+\right\| x_{2} \| \quad\left(=\left\|P_{1} x\right\|+\left\|P_{2} x\right\|\right),
$$

para qualquer $x \in \mathscr{B}$. Como $x=x_{1}+x_{2}$, temos $\|x\| \leq\left\|x_{1}\right\|+\left\|x_{2}\right\|=\|x\|_{1}$. Não é difícil de ver que 
o fato dos espaços $\mathscr{B}_{1}$ e $\mathscr{B}_{2}$ serem completos implica que o espaço $\mathscr{B}$ é completo na norma $\|x\|_{1}$. Desta forma, o Corolário 1.1 implica a existência de uma constante $c>0$ tal que $\|x\|_{1} \leq c\|x\|$,

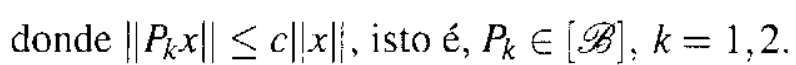

Um operador $P \in\left[\because\left[B^{\circ}\right]\right.$ é chamado de projeção, se $P^{2}=P$. Se um operador $P_{1}$ for uma projeção, então o operador $P_{2}=I-P_{1}$ também será uma projeção e todas as relações de (1.4) serão satisfeitas. Os operadores $P_{1}$ e $P_{2}$ são chamados projeções mutuamente complementares.

Como podemos notar, toda decomposição direta (decomposição em soma direta) de dois subespaços define um par de projeções mutuamente complementares. Reciprocamente, todo par de projeções mutuamente complementares define uma decomposição direta como em (1.2), onde $\mathscr{B}_{1}=P_{1} \mathscr{B}$ e $\mathscr{B}_{2}=P_{2} \mathscr{B}$.

Uma definição análoga existe para a decomposição de um espaço de Banach numa soma direta $\mathscr{B}=\mathscr{B}_{1} \oplus \cdots \in \mathscr{B}_{n}$ de subespaços, e podemos mostrar que toda decomposição direta define e é definida pela decomposição da identidade em soma de projeções $I=P_{1}+\ldots+P_{n}$, as quais são duas a duas disjuntas, ou seja,

$$
P_{j} P_{k}=P_{k} P_{j}=\delta_{j k} P_{k}
$$

onde $\delta_{j k}$ é o símbolo de Kronecker, istoé,

$$
\delta_{j k}=\left\{\begin{array}{cc}
1, & j=k, \\
0, & j \neq k
\end{array}\right.
$$

Notemos, também, que $\|P\| \leq \|\left. P\right|^{2}$, ou seja, $\|P\| \geq 1$ para qualquer projeção $P$. Isto ocorre tendo em vista que $P^{2}=P$

\subsubsection{Complexificação de um Espaço de Banach}

Definimos a complexificação do espaço de Banach $\mathscr{B}$, a qual denotaremos por $\tilde{\mathscr{B}}$ como um espaço de Banach complexo isomorfo ao espaço $\mathscr{B} \times \mathscr{B}$. O espaço $\tilde{\mathscr{B}}$ consiste, por definição, de todos os pares $\left(x_{1}, x_{2}\right)$ de elementos de $\mathscr{B}$ com comportamento algébrico definido pela forma 
simbólica $\left(x_{1}, x_{2}\right)=x_{1}+i x_{2}$ e com norma

$$
\left|x_{1}+i x_{2}\right|=\sqrt{\left|x_{1}\right|^{2}+\left|x_{2}\right|^{2}} \text {. }
$$

Como podemos verificar, $\tilde{\mathscr{B}}$ é um espaço de Banach complexo.

\subsection{Operadores Lineares Limitados}

\subsubsection{Espectro e Resolvente}

Seja $\mathscr{B}$ um espaço de Banach complexo.

Um ponto $\lambda$ do plano complexo será chamado de ponto regular de um operador $A \in[\mathscr{B}]$, se $[\mathscr{B}]$ contiver o operador

$$
R_{\lambda}=(A-\lambda I)^{-1},
$$

que será chamado resolvente de $A$ em $\lambda$.

O conjunto $\rho(A)$ de todos os pontos regulares de um operador $A$ é aberto. O complementar de $\rho(A)$ em $\mathbb{C}$ será chamado espectro de $A$. O espectro de $A$, que denotaremos por $\sigma(A)$, será sempre não-vazio, fechado e estará no disco $|\lambda| \leq\|A\|$.

Teorema 1.5 (Teorema do Raio Espectral) Seja $A \in[\mathscr{B}]$. Então o espectro $\sigma(A)$ está contido no disco de centro na origem e raio

$$
r_{A}=\lim _{n \rightarrow \infty}\left\|A^{n}\right\|^{1 / n}
$$

Observemos que a existência do limite acima segue da relação $\left\|A^{m+n}\right\| \leq\left\|A^{m}\right\|\left\|A^{n}\right\|$.

Demonstração: Notemos que, quando $|\lambda|>r_{A}$, a série $\sum_{k=0}^{\infty} \lambda^{-(k+1)} A^{k}$ converge absolutamente na métrica de $[\mathscr{B}]$. $\Lambda$ série de normas correspondente é majorada pela progressão geométrica $\left\{\left(r_{A}+\varepsilon\right)^{k} /|\lambda|^{k+1}\right\}_{k \in \mathbb{N}}$, para qualquer $\varepsilon>0$ e para $k \in \mathbb{N}$ suficientemente grande. Multiplicando-se 
esta séric por $\lambda I-A$, obtemos $I$. Assim, quando $|\lambda|>r_{A}$, o resolvente sempre existirá e teremos

$$
R_{\lambda}=-\sum_{k=0}^{\infty} \lambda^{(k+1)} A^{k}
$$

Podemos verificar que o círculo $|\lambda|=r_{A}$ sempre contém um ponto do espectro $\sigma(A)$. Portanto o limite $\lim _{n \rightarrow \infty}\left\|A^{n}\right\|^{1 / n}$ será chamado de raio espectral de $A$.

O resolvente $R_{\lambda}$ é uma função analítica de $\lambda$ em uma vizinhança de cada ponto regular $\mu \epsilon$ $\rho(A)$. A expansão absolutamente convergente

$$
R_{\lambda}=R_{\mu}+\sum_{k=0}^{\infty}(\lambda-\mu)^{k} R_{\mu}^{k+1}
$$

é válida para $|\lambda-\mu|<1 /\left\|R_{\mu}\right\|$.

Terminamos esta seção com a seguinte regra especial, notável por sua simplicidade:

Se $A \in[\mathscr{B}]$ for tal que $\|A\|<1$ (ou mais geralmente $r_{A}<1$ ), entāo o operador $I-A$ será invertivel e valerá

$$
(I-A)^{-1}=\sum_{k=0}^{\infty} A^{k}
$$

\subsubsection{A Integral de Riesz}

Sejam $\mathscr{B}$ um espaço de Banach arbitrário e $A \in[\mathscr{B}]$, isto é, $A$ é um operador linear limitado agindo sobre $\mathscr{B}$. Suponhamos que o espectro $\sigma(A)$ de $A$ seja desconexo. Um subconjunto fechado de $\sigma(A)$ cujo complementar em $\sigma(A)$ também é fechado será chamado de conjunto espectral.

Assumiremos que

$$
\sigma(A)=\cup_{k=1}^{m} \sigma_{k}(A)
$$

onde $\sigma_{k}(A), k=1,2, \ldots, m$, são conjuntos espectrais disjuntos. Considerando tal situação, assumiremos que a fronteira $\Gamma_{A}$, onde $\Gamma_{A}$ é um contorno qualquer que cerque o espectro $\sigma(A)$, consiste de partes disjuntas $\Gamma_{k}, k=1,2, \ldots, m$, tais que cada $\Gamma_{k}$ limita o conjunto $G_{k} \supset \sigma_{k}(A)$, sendo que $G_{k}$ é qualquer conjunto que contém $\sigma_{k}(A)$ e não contém nenhum elemento de $\sigma_{i}(A)$, para $i \neq k$. 
Seja

$$
\phi_{k}(\lambda)= \begin{cases}1, & \lambda \in G_{k}, \\ 0, & \lambda \in G_{j}, j \neq k, k=1,2, \ldots, m .\end{cases}
$$

As funções $\phi_{k}(\lambda)$ pertencem à classe de todas as funções a valores complexos $\phi(\lambda)(\lambda$-complexo) analíticas por partes no espectro $\sigma(A)$. Portanto os operadores seguintes são bem definidos

$$
P_{k}=\phi_{k}(A)=-\frac{1}{2 \pi i} \oint_{\Gamma_{A}} \phi_{k}(\lambda) R_{\lambda} d \lambda=-\frac{1}{2 \pi i} \oint_{\Gamma_{k}} R_{\lambda} d \lambda, \quad k=1,2, \ldots, m
$$

O lado direito da igualdade

$$
P_{k}=-\frac{1}{2 \pi i} \oint_{\Gamma_{k}} R_{\lambda} d \lambda
$$

é chamado de integral de Riesz.

Para $\lambda \in G=\cup_{k=1}^{m} G_{k}$, temos

$$
\phi_{k}(\lambda) \phi_{j}(\lambda)=\delta_{k j} \phi_{k}(\lambda)
$$

$\mathrm{e}$

$$
\sum_{k=1}^{m} \phi_{k}(\lambda) \equiv 1
$$

Portanto temos

$$
\begin{aligned}
& P_{k} P_{j}=0, \quad k \neq j ; \\
& P_{k}^{2}=P_{k} \\
& \sum_{k=1}^{m} P_{k}=I .
\end{aligned}
$$

Assim, os operadores $P_{k}$ formam uma decomposição da identidade em uma soma de projeções duas a duas disjuntas. De (1.5) deduzimos que $P_{k} \operatorname{comuta} \operatorname{com} A$, isto é,

$$
P_{k} A=A P_{k}, \quad k=1,2, \ldots, m .
$$

A partir de agora, denotaremos por $P_{k}$ a projeção espectral correspondente a $\sigma_{k}(A)$ como acima.

Sejam $\mathscr{B}_{1}$ um subespaço de $\mathscr{B}$ e $\mathscr{B}=\mathscr{B}_{1} \oplus \mathscr{B}_{2}$. Diremos que $\mathscr{B}_{1}$ é invariante sob um operador 
$A \in[\mathscr{R}]$, se $A \mathscr{B}_{1} \subset \mathscr{B} 1$. Então $\mathscr{B}_{1}$ será invariante com respeito a $A$ se, e somente se,

$$
P A P=A P
$$

onde $P: \mathscr{B} \rightarrow B_{1}$ é uma projeção qualquer.

De (1.7) segue que todo subespaço $\mathscr{B}_{k}=P_{k} \mathscr{B}$ é invariante com respeito a $A$. Também temos que o espectro $\sigma\left(\left.A\right|_{\mathscr{R}_{k}}\right)$ da restrição do operador $A$ ao subespaço $\mathscr{B}_{k}$ coincide com o espectro do conjunto $\sigma_{k}(A)$ e o espaço $\mathscr{B}$ pode ser decomposto como soma direta

$$
\mathscr{B}=\mathscr{B}_{1} \oplus \mathscr{B}_{2} \oplus \cdots \oplus \mathscr{B}_{m}
$$




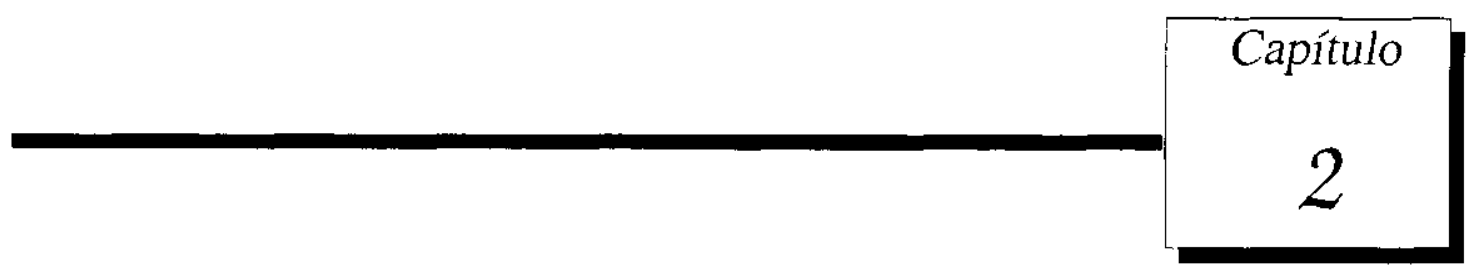

\section{Equações Diferenciais Impulsivas}

Iniciamos este capítulo definindo o que vem a ser uma equação diferencial impulsiva (EDI) e sua solução. Em seguida, enunciamos teoremas de existência e unicidade de soluções e estabelecemos algumas propriedades da matriz de Cauchy de certa EDI linear homogênea. Além disso, apresentamos condições para termos certas desigualdades integrais para funções contínuas por partes.

\subsection{Definições Básicas}

Seja $X=\mathbb{R}^{N}$ um espaço vetorial $N$-dimensional e seja $I: X \rightarrow X$ a aplicação identidade sobre $X$. Denotaremos por $L(X)$ o espaço de todas as matrizes $N \times N$ com entradas em $X$.

Seja $\left\{t_{n}\right\} \subset \mathbb{R}=(-\infty, \infty)$ uma sequuência com índices $\mathrm{cm} \mathbb{Z}$ ou $\mathbb{N}$. Assumiremos que $t_{n}<t_{n+1}$, para todo $n \in \mathbb{Z}$. Além disso, para $\left\{t_{n}\right\}_{n \in \mathbb{N}}$, assumiremos que $t_{n} \rightarrow \infty$ quando $n \rightarrow \infty \mathrm{e}$, para $\left\{t_{n}\right\}_{n \in \mathbb{Z}}$, assumiremos que $t_{n} \rightarrow \pm \infty$ quando $n \rightarrow \pm \infty$. Denotaremos por $J$ um intervalo qualquer de $\mathbb{R}$.

Definição 2.1 Um sistema da forma

$$
\begin{gathered}
\frac{d x}{d t}=f(t, x), \quad t \neq t_{n}, \\
x\left(t_{n}^{\dagger}\right)=x\left(t_{n}\right)+I_{n}\left(x\left(t_{n}\right)\right):=Q_{n}\left(x\left(t_{n}\right)\right),
\end{gathered}
$$


onde

- $f: J \times X \rightarrow X$ é uma função contínua por partes, com descontinuidade de primeira espécie em $t=t_{n}$, contínua à esquerda em $t=t_{n}, n \in \mathbb{Z}$;

- $I_{n}: X \rightarrow X$ é uma função contínua, para todo $n \in \mathbb{Z}$ tal que $t_{n} \in J$.

é dito ser uma equação diferencial impulsiva (escrevemos abreviadamente EDI)

O processo simulado pela EDI (2.1), (2.2) evolui do modo como descrevemos a seguir.

A aplicação $P(t)=(t, x(t))$, que aplica a trajetória de $x(t)$ com ponto inicial $\left(t_{0}, x_{0}\right)$, move-se ao longo da curva $\left\{(t, x): t \geq t_{0}, x=x(t)\right\}$ determinada pela solução da equação diferencial ordinária (escrevemos abreviadamente EDO):

$$
\frac{d x}{d t}=f(t, x)
$$

com valor inicial $x\left(t_{0}\right)=x_{0}, \operatorname{com} t \in\left[t_{0}, t_{1}\right]$. O movimento ao longo desta curva vai até o instante $t_{1}>t_{0}$ no qual a aplicação $P(t)$ encontra o hiperplano $t=t_{1}$. Neste instante, acontece um salto do ponto $\left(t_{1}, x\left(t_{1}\right)\right)$ para o ponto $\left(t_{1}, x\left(t_{1}^{+}\right)\right)$através da ação da função de impulso $I_{1}$. Em seguida, para $t \in\left(t_{1}, t_{2}\right)$, a aplicação $P(t)$ move-se ao longo da curva $\left\{(t, x): t>t_{1}, x=x(t)\right\}$ onde, agora, $x(t)$ é a solução da equação diferencial ordinária (2.3) com $t \in\left[t_{1}, t_{2}\right] \mathrm{e}$ valor inicial $x\left(t_{1}^{+}\right)$. No momento $t=t_{2}$, ocorre uma nova transição por salto realizada do ponto $\left(t_{2}, x\left(t_{2}\right)\right)$ ao ponto $\left(t_{2}, x\left(t_{2}^{+}\right)\right)$pela função de impulso $I_{2}$ e assim por diante.

Definição 2.2 Uma solução da EDI (2.1), (2.2) é qualquer função contínua por partes, $x: J \rightarrow X$, com descontinuidade de primeira espécie em $t=t_{n}$, contínua à esquerda em $t=t_{n}$ e tal que as equaçōes (2.1) e (2.2) estejam satisfeitas.

Denotaremos por $x\left(t ; t_{0}, x_{0}\right)=x(t)$ a solução da EDI (2.1), (2.2) para a qual $x\left(t_{0}\right)=x_{0} \mathrm{e}$ por $\Omega_{+}$(respectivamente $\Omega_{-}$) o intervalo maximal no qual esta solução é contínua à direita (respectivamente à esquerda) do instante $t_{0}$. Veremos, na próxima seção, que um tal intervalo existe.

Se $x(t)=x\left(t ; t_{0}, x_{0}\right)$ for uma solução da EDI (2.1), (2.2) com condição inicial $x\left(t_{0}\right)=x_{0}$, então 
a seguinte representação será válida

$$
x(t)= \begin{cases}x_{0}+\int_{t_{0}}^{t} f(s, x(s)) d s+\sum_{t_{0}<t_{n}<t} I_{n}\left(x\left(t_{n}\right)\right), & t \in \Omega_{+}, \\ x_{0}+\int_{t_{0}}^{t} f(s, x(s)) d s-\sum_{i<t_{n}<t_{0}} I_{n}\left(x\left(t_{n}\right)\right), & t \in \Omega_{-} .\end{cases}
$$

\subsection{Existência e Unicidade de Soluções}

O problema de existência e unicidade da solução da EDI (2.1), (2.2) pode ser reduzido ao problema de existência e unicidade da EDO (2.1) correspondente, considerando-se cada intervalo $\left[t_{n-1}, t_{n}\right], n \in \mathbb{Z}$.

Teorema 2.1 [[1], Teorema 2.1] O problema de valor inicial para a EDI (2.1), (2.2) com valor inicial $\left(t_{0}, x_{0}\right)$ tem solução única à direita de $t_{0}$ se, e somente se, a EDO (2.3) tiver solução única para $t_{0} \neq t_{n}$ e $x_{0} \in X$ ou se $t_{0}=t_{n}$ e $x_{0}=x\left(\iota_{n}^{i}\right)$, para algum $n \in \mathbb{Z}$. O problema de valor inicial para a EDI (2.1), (2.2) com valor inicial $\left(t_{0}, x_{0}\right)$ tem solução única à esquerda de $t_{0}$ se, e somente se, existirem funções $I_{n}^{-1}, n \in \mathbb{Z}$, e a equação diferencial ordinária (2.3) tiver solução única para $t_{0} \in \mathbb{R} e x_{0} \in X$.

Consideremos a EDI linear homogênea

$$
\begin{gathered}
\frac{d x}{d t}=A(t) x, \quad t \neq t_{n}, \\
x\left(t_{n}^{+}\right)=x\left(t_{n}\right)+I_{n}\left(x\left(t_{n}\right)\right):=Q_{n}\left(x\left(t_{n}\right)\right),
\end{gathered}
$$

onde

- $A: J \rightarrow X$ é uma função contínua por partes, com descontinuidade de primeira espécie em $t=t_{n}$, contínua à esquerda em $t=t_{n}, n \in \mathbb{Z}$

- $I_{n}: X \rightarrow X$ é uma função contínua, para todo $n \in \mathbb{Z}$ tal que $t_{n} \in J$. 
O teorema a seguir é uma conseqüência imediata do Teorema de Existência e Unicidade para EDOs aplicado a cada intervalo $\left[t_{n}, t_{n+1}\right]$, para $n \in \mathbb{Z}$.

Teorema 2.2 [I]], Teorema 2.2] Dado qualquer ponto $\left(t_{0}, x_{0}\right) \in J \times X$, a EDI (2.4), (2.5) admite uma solução única $x(t)$ definida para todo $t \geq t_{0}$ e tal que $x\left(t_{0}\right)=x_{0}$.

Definição 2.3 Uma funçāo a valores matriciais $U(t, \tau)$ que associa a cada elemento $x_{\tau} \in X, \tau \leq t$, uma única solução determinada pelo problema de valor inicial (2.4), $(2.5), x(\tau)=x_{\tau}$, isto é,

$$
U(t, \tau) x_{\tau}=x(t)
$$

é uma matriz ou operador de Cauchy da EDI (2.4), (2.5). Em particular, quando $\tau=t_{0}$, temos $x_{\tau}=x_{0}$ e, neste caso, escrevemos $U(t)$ em lugar de $U\left(t, t_{0}\right)$ e dizemos que $U(t)$ é a matriz ou operador fundamental da EDI (2.4), (2.5) $\operatorname{com} x\left(t_{0}\right)=x_{0}$.

Supondo que as matrizes $Q_{n}$, em (2.5) sejam não-singulares, isto é, det $Q_{n} \neq 0, n \in \mathbb{Z}$, podemos ver que os operadores $U(t)$ e $U(t, \tau)$ satisfazem

$$
U(t)=X\left(t, t_{n}\right) Q_{n} X\left(t_{n}, t_{n-1}\right) Q_{n-1} \ldots Q_{1} X\left(t_{1}, t_{0}\right)
$$

$$
U(t, s)= \begin{cases}X_{k}(t, s), & s, t \in\left[t_{k-1}, t_{k}\right], \\ X_{k+1}\left(t, t_{k}\right) Q_{k} X_{k}\left(t_{k}, s\right), & t_{k-1}<s \leq t_{k}<t \leq t_{k+1}, \\ X_{k+1}\left(t, t_{k}\right) Q_{k}^{-1} X_{k}\left(t_{k}, s\right), & t_{k-1}<t \leq t_{k}<s \leq t_{k+1} . \\ X_{k+1}\left(t, t_{k}\right) \prod_{j=k}^{i+1}\left[Q_{j} X_{j}\left(t_{j}, t_{j-1}\right)\right] Q_{i} X_{i}\left(t_{i}, s\right), & t_{i-1}<s \leq t_{i}<t_{k}<t \leq t_{k+1}, \\ X_{i}\left(t, t_{i}\right) \prod_{j=i}^{k-1}\left[Q_{j}^{-1} X_{j}\left(t_{j}, t_{j-1}\right)\right] Q_{k}^{-1} X_{k \vdash 1}\left(t_{k}, s\right), & t_{i-1}<t \leq t_{i}<t_{k}<s \leq t_{k+1},\end{cases}
$$

onde $X_{k}(t, \tau)$ é a matriz de Cauchy da equação diferencial ordinária

$$
\frac{d x}{d t}=A(t) x
$$


restrita ao intervalo $\left[t_{k}, t_{k \cdot 1}\right]$, com condição inicial $x\left(t_{k}^{+}\right)$, para $k \in \mathbb{Z}$.

Notemos que

$$
\begin{array}{ll}
U(t, t)=I, & \\
U(t, s)=U(t, \tau) U(\tau, s), & \tau \leq s \leq t, \\
\frac{d}{d t} U(t, \tau)=A(t) U(t, \tau), & \tau \leq t, t \notin\left\{t_{n}\right\}, \\
\frac{d}{d \tau} U(t, \tau)=-U(t, \tau) A(\tau), & \tau \leq t, t \notin\left\{t_{n}\right\}, \\
U\left(t_{n}^{+}, \tau\right)=Q_{n} U\left(t_{n}, \tau\right), & \tau \leq t_{n}, \\
U\left(t, t_{n}^{+}\right)=U\left(t, t_{n}\right) Q_{n}^{-1}, & t_{n}<t .
\end{array}
$$

Agora, vamos considerar a EDI linear não-homogênea

$$
\begin{gathered}
\frac{d x}{d t}=A(t) x+f(t, x), \quad t \neq t_{n}, \\
x\left(t_{n}^{+}\right)=x\left(t_{n}\right)+I_{n}\left(x\left(t_{n}\right)\right)+h_{n}(x)=Q_{n}\left(x\left(t_{n}\right)\right)+h_{n}(x),
\end{gathered}
$$

onde

- $A: J \rightarrow X$ é uma função contínua por partes, com descontinuidade de primeira espécie em $t=t_{n}$, contínua à esquerda em $t=t_{n}, n \in \mathbb{Z}$;

- $f: J \times X \rightarrow X$ é uma função contínua por partes, com descontinuidade de primeira espécie em $t=t_{n}$, contínua à esquerda em $t=t_{n}, n \in \mathbb{Z}$;

- $I_{n}: X \rightarrow X$ é uma função contínua, para todo $n \in \mathbb{Z}$ tal que $t_{n} \in J$.

O lema a seguir é conhecido como Fórmula da Variação das Constantes para EDIs c [1] o apresenta separadamente em dois lemas. Este resultado apresenta a solução da EDI não-homogênea (2.7), (2.8) utilizando o operador de Cauchy da EDI homogênea associada a ela, isto é, a EDI (2.1), (2.2).

Lema 2.1 (Fórmula da Variação das Constantes para EDIs) [/ I], Lemas 2.2 e 2.3] Consideremos a EDI (2.7), (2.8) e suponhamos que a função $f(t, x)$ seja localmente lipschitziana em $x$. Seja 
$x(t)=x\left(t ; t_{0}, x_{0}\right)$ uma solução da EDI não-homogênea $(2.7),(2.8)$. Então

$$
x(t)=U\left(t, t_{0}^{+}\right) x_{0}+\int_{t_{0}}^{t} U(t, s) f(s, x(s)) d s+\sum_{t_{0}<t_{n}<t} U\left(t, t_{n}^{\dagger}\right) h_{n}\left(x\left(t_{n}\right)\right), \quad t \in \Omega_{+} .
$$

Se, além disso, as matrizes $Q_{n}$ forem invertíveis para todo $n \in \mathbb{Z}$, então

$$
x(t)=U\left(t, t_{0}^{+}\right) x_{0}+\int_{t_{0}}^{t} U(t, s) f(s, x(s)) d s-\sum_{t<t_{n}<t_{0}} U\left(t, t_{n}^{-}\right) h_{n}\left(x\left(t_{n}\right)\right), \quad t \in \Omega_{-},
$$

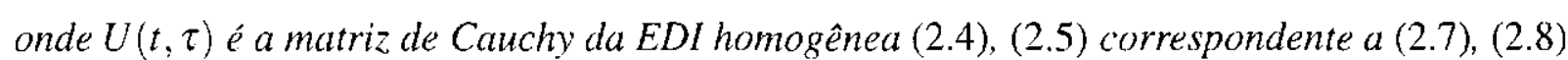

\subsection{Desigualdades Integrais}

Nesta seção, provamos algumas desigualdades integrais que serão usadas no decorrer do texto.

Lema 2.2 [[2], Teorema 1.4.1] Suponhamos que $\varphi:\left[t_{0},+\infty\right) \rightarrow \mathbb{R}$ seja uma função não-negativa, contínua por partes com descontinuidade de primeira espécie nos pontos $t_{n} \in\left[t_{0},+\infty\right), n \in \mathbb{N}$ Sejam $\mu, v:\left[t_{0},+\infty\right) \rightarrow \mathbb{R}$ funçóes contínuas não-negativas e suponhamos que $\beta_{n} \geq 0$ e $\gamma_{n}$ sejam constantes para cada $n \in \mathbb{N}$. Suponhamos, ainda, que

$$
\varphi^{\prime}(t) \leq v(t) \varphi(t)+\mu(t)
$$

eque

$$
\varphi\left(t_{n}^{+}\right) \leq \beta_{n} \varphi\left(t_{n}\right)+\gamma_{n}, \quad n \in \mathbb{N} .
$$

Então

$$
\begin{aligned}
\varphi(t) \leq & \varphi\left(t_{0}\right) \prod_{t_{0}<t_{n}<t} \beta_{n} e^{\int_{t_{0}}^{t} v(\tau) d \tau}+\sum_{t_{0}<t_{n}<t}\left(\prod_{t_{n}<t_{i}<t} \beta_{i} e^{\int_{t_{n}}^{t} v(\tau) d \tau}\right) \gamma_{n}+ \\
& +\int_{t_{0}}^{t}\left(\prod_{\tau<t_{i}<t} \beta_{i} e^{f_{\tau}^{t} v(s) d s}\right) \mu(\tau) d \tau, \quad t \geq t_{0} .
\end{aligned}
$$

Demonstração: Provemos por indução sobre os intervalos $\left[t_{0}, t_{n}\right], n \in \mathbb{N}$. 
Scja $t \in\left[t_{0}, t_{1}\right]$. Então, de (2.11), temos

$$
\frac{d}{d t}\left[\varphi(t) e^{-\int_{t_{0}}^{t} v(s) d s}\right] \leq \mu(t) e^{-\int_{t_{0}}^{t} v(s) d s}
$$

que integrando de $t_{0}$ a $t, t_{0} \leq t \leq t_{1}$, implica $\mathrm{cm}$

$$
\varphi(t) \leq \varphi\left(t_{0}\right) e^{\int_{t_{0}}^{t} v(s) d s}+\int_{t_{0}}^{t} \mu(\tau) e^{\int_{\tau}^{t} v(s) d s} d \tau
$$

Portanto (2.13) é verdadeira para $t \in\left[t_{0}, t_{1}\right]$.

Agora, suponhamos que (2.13) seja válida para $t \in\left[t_{0}, t_{n}\right]$ para algum inteiro positivo $n \geq 1$. Então, para $t \in\left[t_{0}, t_{n-1}\right]$, segue de (2.11) e (2.14) que

$$
\varphi(t) \leq \varphi\left(t_{n}^{+}\right) e^{\int_{t_{n}}^{t} v(s) d s}+\int_{l_{n}}^{t} \mu(\tau) e^{f_{\tau}^{t} v(s) d s} d \tau
$$

e, usando (2.12), obtemos

$$
\varphi(t) \leq\left(\beta_{n} \varphi\left(t_{n}\right)+\gamma_{n}\right) e^{\int_{t_{n}}^{t} v(s) d s}+\int_{t_{n}}^{t} \mu(\tau) e^{\int_{\tau}^{t} v(s) d s} d \tau
$$

Pela hipótese de indução, (2.15) implica que

$$
\begin{aligned}
\varphi(t) \leq & \beta_{n} e^{\int_{t_{n}}^{t_{n}} v(s) d s}\left[\varphi\left(t_{0}\right) \prod_{t_{0}<t_{k}<t_{n}} \beta_{k} e^{\int_{t_{0}}^{t_{0}} v(s) d s}+\sum_{t_{0}<t_{k}<t_{n}}\left(\prod_{t_{k}<t_{i}<t_{n}} \beta_{i} e^{\int_{t_{k}}^{t_{n}} v(s) d s}\right) \gamma_{k}+\right. \\
& \left.+\int_{t_{0}}^{t_{n}}\left(\prod_{\tau<t_{i}<t_{n}} \beta_{i} e^{\int_{\tau}^{t_{\tau}} v(s) d s}\right) \mu(\tau) d \tau\right]+\gamma_{n} e^{\int_{t_{n}}^{t} v(s) d s}+\int_{t_{n}}^{t} \mu(\tau) e^{f_{\tau}^{t} v(s) d s} d \tau .
\end{aligned}
$$

Portanto

$$
\begin{aligned}
\varphi(t) \leq & \varphi\left(t_{0}\right) \prod_{t_{0}<t_{n}<t} \beta_{n} e^{\int_{t_{0}}^{t} v(\tau) d \tau}+\sum_{t_{0}<t_{n}<t}\left(\prod_{t_{n}<t_{i}<t} \beta_{i} e^{f_{t_{n}}^{t} v(\tau) d \tau}\right) \gamma_{n}+ \\
& +\int_{t_{0}}^{t}\left(\prod_{\tau<t_{i}<t} \beta_{i} e^{f_{\tau}^{t} v(s) d s}\right) \mu(\tau) d \tau
\end{aligned}
$$

para $t \in\left[t_{0}, t_{n+1}\right]$. Isto completa a prova.

Lema 2.3 [/ 1], Lema 3.1] Suponhamos que $\varphi:\left[t_{0}, \infty\right) \rightarrow \mathbb{R}$ seja uma função não-negativa, contínua por partes com descontinuidade de primeira espécie nos pontos $t_{i}$, para todo $i \in \mathbb{N}$. Seja 
$v:\left[t_{0}, \infty\right) \rightarrow \mathbb{R}$ uma função contínua não-negativa e sejam $c \geq 0$ e $\beta_{i} \geq 0, i \in \mathbb{N}$, constantes. Suponhamos, ainda, que

$$
\varphi(t) \leq c+\sum_{t_{0}<t_{i}<i} \beta_{i} \varphi\left(t_{i}\right)+\int_{t_{0}}^{t} \varphi(\tau) v(\tau) d \tau, \quad t_{0} \geq 0
$$

Entāo

$$
\varphi(t) \leq c \prod_{t_{0}<t_{i}<t}\left(1+\beta_{i}\right) e^{\int_{t_{0}}^{t} v(\tau) d \tau}
$$

Demonstração: Consideremos $\psi:\left[t_{0}, \infty\right) \rightarrow \mathbb{R}$ dada por

$$
\psi(t)=c+\sum_{t_{0}<t_{i}<t} \beta_{i} \varphi\left(t_{i}\right)+\int_{t_{0}}^{t} \varphi(\tau) v(\tau) d \tau
$$

Logo, para $t \geq t_{0}$, temos

$$
\begin{gathered}
\psi^{\prime}(t)=v(t) \varphi(t), \quad t \neq t_{n}, \\
\psi\left(t_{0}\right)=c \\
\psi\left(t_{n}^{+}\right) \leq\left(1+\beta_{n}\right) \varphi\left(t_{n}\right) .
\end{gathered}
$$

Como $\varphi(t) \leq \psi(t)$, dado $t \in\left[t_{0}, \infty\right)$, temos

$$
\psi^{\prime}(t) \leq v(t) \psi(t), \quad t \neq t_{n}
$$

Além disso,

$$
\psi\left(t_{0}\right)=c
$$

$\mathrm{c}$

$$
\psi\left(t_{n}^{+}\right) \leq\left(1+\beta_{n}\right) \psi\left(t_{n}\right), \quad n \in \mathbb{N} .
$$

Deste modo, podemos aplicar o Lema 2.2 para obtermos

$$
\psi(t) \leq c \prod_{t_{0}<t_{i}<t}\left(1+\beta_{i}\right) e^{\int_{t_{0}}^{t} v(\tau) d \tau}, \quad t \geq t_{0}
$$

e, portanto, o resultado está provado. 
Denotemos por $i(a, b)$ o número de pontos da seqüência $\left\{t_{n}\right\}_{n \in \mathbb{Z}}$ contidos no interior de certo intervalo $[a, b] \subset \mathbb{R}$ e, por $j(t)=i\left(t_{0}, t\right)$. Deste modo, $j(t)=n$, se $t_{n}<t \leq t_{n+1}, n \in \mathbb{Z}$.

O próximo lema é um caso particular do Lema 2.3.

Lema 2.4 [/1], Lema 3.2] Suponhamos que $\varphi:\left[t_{0}, \infty\right) \rightarrow \mathbb{R}$ seja uma função não-negativa, contínua por partes com descontinuidade de primeira espécie nos pontos $t_{i}$, para todo $i \in \mathbb{N}$, e que

$$
\varphi(t) \leq \alpha+\sum_{t_{0}<t_{i}<t} \beta \varphi\left(t_{i}\right)+\int_{t_{0}}^{t} \gamma \varphi(\tau) d \tau
$$

onde $\alpha, \beta \geq 0$ e $\gamma>0$ são constantes. Então

$$
\varphi(t) \leq \alpha(1+\beta)^{i\left(t_{0}, t\right)} e^{\gamma\left(t-t_{0}\right)}
$$

Lema 2.5 [[1], Lema 3.3] Suponhamos que $\varphi:\left[t_{0}, \infty\right) \rightarrow \mathbb{R}$ seja uma função não-negativa, contínua por partes com descontinuidade de primeira espécie nos pontos $t_{i}$, para todo $i \in \mathbb{N}$ tal que, para $t \geq t_{0}$, tenhamos

$$
\varphi(t) \leq \alpha+\int_{t_{0}}^{t}[\beta+\gamma \varphi(\tau)] d \tau+\sum_{t_{0}<t_{i}<t}\left[\beta+\gamma \varphi\left(t_{i}\right)\right],
$$

onde $\alpha, \beta \geq 0$ e $\gamma>0$ são constantes. Então

$$
\varphi(t) \leq\left(\alpha+\frac{\beta}{\gamma}\right)(1+\gamma)^{i\left(t_{0} l t\right)} e^{\gamma\left(t-t_{0}\right)}-\frac{\beta}{\gamma} .
$$

Demonstração: Note que (2.17) pode ser reescrita do seguinte modo

$$
\varphi(t)+\frac{\beta}{\gamma} \leq \alpha+\frac{\beta}{\gamma}+\int_{t_{0}}^{t} \gamma\left[\varphi(\tau)+\frac{\beta}{\gamma}\right] d \tau+\sum_{t_{0}<t_{i}<t} \gamma\left[\varphi\left(t_{i}\right)+\frac{\beta}{\gamma}\right]
$$

Assim, a função $\varphi(t)+\frac{\beta}{\gamma}$ satisfaz as condições do Lema 2.4. Portanto,

$$
\varphi(t)+\frac{\beta}{\gamma} \leq\left(\alpha+\frac{\beta}{\gamma}\right)(1+\gamma)^{i\left(t_{0}, t\right)} e^{\gamma\left(t-t_{0}\right)}
$$

e a demonstração está completa 
Consideremos, agora, a EDI linear não-homogênea

$$
\begin{gathered}
\frac{d x}{d t}=A(t) x+f(t), \quad t \neq t_{n}, \\
x\left(t_{n}^{\dagger}\right)=x\left(t_{n}\right)+I_{n}\left(x\left(t_{n}\right)\right)+h_{n}=Q_{n}\left(x\left(t_{n}\right)\right)+h_{n} .
\end{gathered}
$$

onde

- $A: \mathbb{R}_{+} \rightarrow L(X)$ é uma função contínua por partes, com descontinuidade de primeira espécie em $t=t_{n}$, contínua à esquerda em $t=t_{n}, n \in \mathbb{N}$;

- $f: \mathbb{R}_{+} \rightarrow X$ é uma função contínua por partes, com descontinuidade de primeira espécie em $t=t_{n}$, contínua à esquerda em $t=t_{n}, n \in \mathbb{N}$;

- $I_{n}: X \rightarrow X$ é uma função contínua, para qualquer $n \in \mathbb{N}$;

- $h_{n} \in X$, para qualquer $n \in \mathbb{N}$.

O próximo lema nos dá uma estimativa para a solução da EDI (2.18), (2.19).

Lema 2.6 [[1], Lema 3.5] Consideremos a EDI não-homogênea (2.18), (2.19) e seja $\tilde{J} \subset \mathbb{R}$ um intervalo de comprimento l. Então, para qualquer solução $x(t)$ de (2.18), (2.19), teremos

$$
\begin{array}{r}
|x(t)| \leq\left(\left|x\left(t_{0}\right)\right|+\int_{\tilde{J}}|f(u)| d u+\sum_{t_{k} \in \tilde{I}}\left|h_{k}\right|\right) e^{\int_{j}|A(u)| d u} \prod_{l_{n} \in \tilde{J}}\left(1+\left\|I_{n}\right\|\right) \\
x(t) \mid \leq\left(l^{-1} \int_{\tilde{J}}\left|x(u)_{i} d u+\int_{\tilde{J}}\right| f(u)^{\prime} d u+\sum_{t_{k} \in \tilde{J}}\left|h_{k}\right|\right) e^{\int_{j} \mid A(u) ; d u} \prod_{t_{n} \in \tilde{J}}\left(1+\| I_{n}||\right) .
\end{array}
$$

Demonstração: Pelas fórmulas (2.9) e (2.10) para $x(t)$, temos

$$
x(t)= \begin{cases}x_{0}+\int_{t_{0}}^{t} f(u) d u+\int_{t_{0}}^{t} A(u) x(u) d u+\sum_{t_{0}<t_{n}<t} h_{n}+\sum_{t_{0}<t_{n}<t} I_{n} x\left(t_{n}\right), & t>t_{0}, \\ x_{0}+\int_{t_{0}}^{t} f(u) d u+\int_{t_{0}}^{t} A(u) x(u) d u-\sum_{i<t_{n}<t_{0}} h_{n}-\sum_{t<t_{n}<t_{0}} I_{n} x\left(t_{n}\right), & t<t_{0} .\end{cases}
$$


Então

$$
|x(t)| \leq\left|x_{0}\right|+\left|\int_{t_{0}}^{t}\right| f(u)|d u|+\left|\int_{t_{0}}^{t}\right| A(u)|||x(u)| d u\left|+\sum_{t_{n} \in \Omega}\right| h_{n}\left|+\sum_{t_{n} \in \Omega} \| I_{n}\right||| x\left(t_{n}\right) \mid,
$$

onde

$$
\Omega= \begin{cases}\left(t_{0}, t\right), & t \geq t_{0} \\ \left(t, t_{0}\right), & t<t_{0}\end{cases}
$$

Então

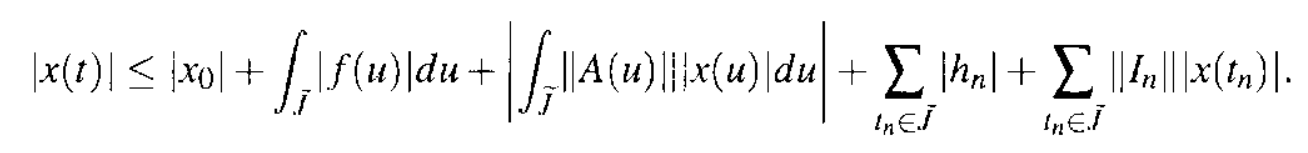

Daí, pelo Lema 2.3, segue a desigualdade (2.20).

Provemos, agora, a desigualdade (2.21). Para isto, integramos a desigualdade (2.22) sobre $\tilde{J} \mathrm{e}$ obtemos

$$
|x(t)| l \leq e^{\int_{j, A} A(u) \mid, d u} \prod_{t_{n} \in \tilde{J}}\left(1+\left\|I_{n}\right\|\right)\left(\int_{\tilde{J}}|x(u)| d u+l \int_{\tilde{J}}|f(u)| d u+l \sum_{t_{n} \in \tilde{J}}\left|h_{n}\right|\right) .
$$

A estimativa (2.21) segue de (2.23).

Observação 2.1 Em particular, se x(t) for uma solução da EDI homogênea (2.4), (2.5) e $t_{0}, t \in \tilde{J}$, então teremos as seguintes estimativas para $|x(t)|$ :

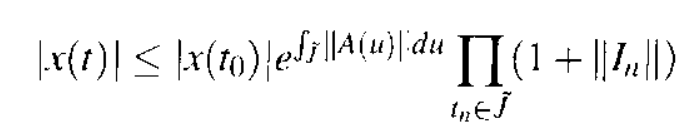

e

$$
|x(t)| \leq \frac{1}{l}\left(\int_{\tilde{J}}|x(u)| d u\right) e^{\int_{J}\|A\langle u)\| d u} \prod_{t_{n} \in \tilde{J}}\left(1+\left\|I_{n}\right\|\right) .
$$

Para isto, basta aplicarmos o Lema 2.6 com $f=0$ e $h_{n}=0, n \in \mathbb{N}$.

O próximo lema é a Desigualdade de Gronwall Generalizada que será utilizada no último capítulo desta dissertação. 
Lema 2.7 (Desigualdade de Gronwall Generalizada) /[5], Lema 6.2] Suponhamos que $\alpha, \beta, \phi$ : $[a, b] \rightarrow \mathbb{R}$ sejam funções contínuas, com $\beta(t) \geq 0$, para todo $t \in[a, b]$, tais que

$$
\phi(t) \leq \alpha(t)+\int_{a}^{t} \beta(s) \phi(s) d s, \quad t \in[a, b]
$$

Então

$$
\phi(t) \leq \alpha(t)+\int_{a}^{t} \beta(s) \alpha(s) e^{\int_{s}^{t} \beta\{u) d u} d s, \quad t \in[a, b]
$$

Demonstração: Definamos

$$
V(\tau)=\int_{a}^{\tau} \beta(s) \phi(s) d s, \quad \tau \in[a, b]
$$

Dado $\tau \in[a, b]$, temos

$$
\frac{d V(\tau)}{d \tau}=\beta(\tau) \phi(\tau) \leq \beta(\tau) \alpha(\tau)+V(\tau) \beta(\tau)
$$

daí

$$
\frac{d V(\tau)}{d \tau}-V(\tau) \beta(\tau) \leq \beta(\tau) \alpha(\tau)
$$

Multiplicando ambos os lados da desigualdade por $e^{-\int_{a}^{\tau} \beta(s) d s}$ segue que

$$
e^{-\int_{a}^{\tau} \beta(s) d s} \frac{d V(\tau)}{d \tau}-V(\tau) \beta(\tau) e^{-\int_{a}^{\tau} \beta(s) d s} \leq \beta(\tau) \alpha(\tau) e^{-\int_{a}^{\tau} \beta(s) d s},
$$

donde

$$
\frac{d}{d \tau}\left(V(\tau) e^{-\int_{a}^{\tau} \beta(s) d s}\right) \leq \beta(\tau) \alpha(\tau) e^{-\int_{a}^{\tau} \beta(s) d s}
$$

Integrando de $a$ a $t$ temos

$$
V(t) e^{-\int_{a}^{t} \beta(s) d s} \leq \int_{a}^{t} \beta(\tau) \alpha(\tau) e^{-\int_{a}^{\tau} \beta(s) d s} d \tau
$$

Portanto, para $t \in[a, b]$, temos

$$
V(t) \leq \int_{a}^{t} \beta(\tau) \alpha(\tau) e^{\int_{\tau}^{t} \beta(s) d s} d \tau .
$$


Como $\phi(t) \leq \alpha(t)+V(t)$, segue, para $t \in[a, b]$, que

$$
\phi(t) \leq \alpha(t)+\int_{a}^{t} \beta(s) \alpha(s) e^{\int_{s}^{t} \beta(u) d u} d s .
$$

Portanto, a Desigualdade de Gronwall Generalizada está demonstrada. 


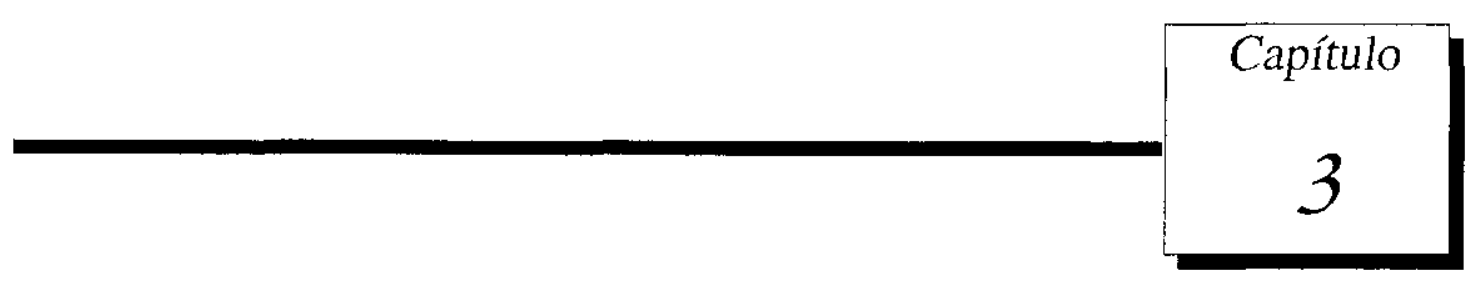

Dicotomias para Equações Diferenciais

Impulsivas

Neste capítulo, apresentamos as definições de vários tipos de dicotomia para certa EDI linear homogênea. Definimos expoente geral superior $\mathfrak{e}$ inferior e aprescntamos uma relação entre estes expoentes e a existência de uma dicotomia espectral. Finalmente, definimos equivalência cinemática e apresentamos propriedades fundamentais deste conceito para EDIs.

\subsection{Definições e Propriedades}

Seja $J \subset \mathbb{R}$ um intervalo qualquer. Consideremos a EDI linear homogênea

$$
\begin{gathered}
\frac{d x}{d t}=A(t) x, \quad t \neq t_{n}, \\
x\left(t_{n}^{\dagger}\right)=x\left(t_{n}\right)+I_{n}\left(x\left(t_{n}\right)\right)=Q_{n}\left(x\left(t_{n}\right)\right),
\end{gathered}
$$

onde

- $A: J \subset \mathbb{R} \rightarrow L(X)$ é uma função contínua por partes, com descontinuidade de primeira espécie em $t=t_{n}$, contínua à esquerda em $t=t_{n}$, para qualquer $n \in \mathbb{Z}$ tal que $t_{n} \in J$; 
- $I_{n}: X \rightarrow X$ é um operador contínuo, para qualquer $n \in \mathbb{Z}$ tal que $t_{n} \in J$.

Definimos $Q(t)$ pela fórmula

$$
Q(t)=\sum_{t_{n} \in I} Q_{n} \delta\left(t-t_{n}\right)
$$

$\operatorname{com} \delta(t)$ sendo a função característica do conjunto $\{0\}$. Esta função $Q$ será importante ao tratarmos de funções quase periódicas.

Vamos introduzir alguns conceitos essenciais para a obtenção dos resultados deste capítulo.

Definição 3.1 Seja $X$ um espaço de Banach e sejam $X_{1}$ e $X_{2}$ subespaços de $X$ com $X=X_{1} \oplus X_{2}$. Introduzimos a seguinte caracterização de suas inclinações mútuas:

$$
S_{n}\left(X_{1}, X_{2}\right)=\inf \left\{\left|x_{1}+x_{2}\right|: x_{i} \in X_{i},\left|x_{i}\right|=1, i=1,2\right\} .
$$

Definição 3.2 Consideremos a $E D I$ (3.1), (3.2) e suponhamos que $\operatorname{det} Q_{n} \neq 0$, para qualquer $n \in \mathbb{Z}$ tal que $t_{n} \in J$. Diremos que (3.1), (3.2) tem uma dicotomia exponencial, se o espaço $X=\mathbb{R}^{N}$ puder ser decomposto em uma soma direta de dois subespaços $X_{1}$ e $X_{2}$, isto é, $X=X_{1} \oplus X_{2}$, e as seguintes condiçōes forem satisfeitas:

(i) para $s, t \in J$, com $s \leq t$,

$$
\left|x_{1}(t)\right| \leq N_{1} e^{-v_{1}(t-s)}\left|x_{1}(s)\right|
$$

onde $x_{1}(t)=U\left(t, t_{0}\right) x_{0}^{1} \in X_{1}$ e $N_{1}, v_{1}>0$

(ii) para $s, t \in J$, com $s \geq t$,

$$
\left|x_{2}(t)\right| \leq N_{2} e^{-v_{2}(s-t)}\left|x_{2}(s)\right|
$$

onde $x_{2}(t)=U\left(t, t_{0}\right) x_{0}^{2} \in X_{2}$ e $N_{2}, v_{2}>0$;

(iii) para qualquer $t \in J$

$$
S_{n}\left(U(t) X_{1}, U(t) X_{2}\right) \geq \gamma>0,
$$

onde $t_{0} \in J$ é fixo, $U(t, s)$ é a matriz de Cauchy de (3.1), (3.2) e $U(t)=U(t, 0)$. 
Definição 3.3 Temos as seguintes definições:

(i) Chamamos de expoente geral superior de uma solução $x(t)$ da EDI (3.1), (3.2) o seguinte número

$$
\inf \left\{\alpha \in \mathbb{R}: \exists N_{\alpha}>0 \text { tal que }|x(t)| \leq N_{\alpha} e^{\alpha(t-s)}|x(s)|, \forall s \leq t, s, t \in J\right\}
$$

que será denotado por $g(x(t))$.

(ii) Chamamos de expoente geral superior das soluções da EDI (3.1), (3.2), iniciando no subespaço $X_{1} \subset X$ no momento $t_{0} \in J$, o seguinte número

$$
\begin{aligned}
\inf \{\alpha \in \mathbb{R}: \quad & \exists N_{\alpha}>0 \text { tal que }\left|x(t) ! \leq N_{\alpha} e^{\alpha(t-s)}\right| x(s) \mid, \\
& \text { parax } \left.x(t)=U\left(t, t_{0}\right) x_{0}^{1}, \forall x_{0}^{1} \in X_{1}, \forall s \leq t, s, t \in J\right\},
\end{aligned}
$$

que denotaremos por $g\left(X_{1}\right)$.

(iii) Chamamos de expoente geral inferior de uma solução $x(t)$ da EDI (3.1), (3.2) o seguinte , número

$$
\sup \left\{\alpha \in \mathbb{R}: \exists N_{\alpha}>0 \text { tal que }|x(t)| \geq N_{\alpha} e^{\alpha(t-s)}|x(s)|, \forall s \geq t, s, t \in J\right\},
$$

que será denotado por $g^{\prime}(x(t))$.

(iv) Chamamos de expoente geral inferior das soluções da EDI (3.1), (3.2), iniciando no subespaço $X_{1} \subset X$ no momento $t_{0} \in J$, o seguinte número

$$
\begin{aligned}
\sup \{\alpha \in \mathbb{R}: \quad & \exists N_{\alpha}>0 \text { tal que }|x(t)| \geq N_{\alpha} e^{\alpha(t-s)}|x(s)|, \\
& \left.\operatorname{parax}(t)=U\left(t, t_{0}\right) \cdot x_{0}^{1}, \forall x_{0}^{1} \in X_{1}, \forall s>t, s, t \in J\right\} .
\end{aligned}
$$

Denotaremos este número por $g^{\prime}\left(X_{1}\right)$.

Observação 3.1 Podemos notar que as desigualdades (3.4), (3.5) da Definição 3.2 são equiva- 
lentes, respectivamente, às seguintes desigualdades

$$
g\left(X_{1}\right)<0 \quad \text { e } \quad g^{\prime}\left(X_{2}\right)<0
$$

Lema 3.1 Suponhamos que U seja a matriz fundamental da $E D I(3.1),(3.2)$ e que $\operatorname{det} Q_{n} \neq 0$, para qualquer $n \in \mathbb{Z}$ tal que $t_{n} \in J$. Então, para quaisquer $s, t \in\left[t_{j}, t_{k}\right] \subset J$, teremos

$$
\|U(t, s)\| \leq M_{1} e^{\int_{t j}^{t_{j}}|: A(u)| d u},
$$

onde $M_{1}=(k-j) \max \left\{\left\|Q_{i}\right\|,\left\|Q_{i}^{-1}\right\| ; i=j+1,2, \ldots, k\right\}$.

Demonstração: Sejam $s, t \in\left[t_{j}, t_{k}\right]$. Se $s \leq t$, então existem inteiros $l$ e $m$, com $j \leq l \leq m \leq k$, tais que

$$
t_{l} \leq s \leq t_{l+1} \quad \text { e } \quad t_{n} \leq t \leq t_{m+1}
$$

Então

$$
U(t, s)=U\left(t, t_{m}\right) Q_{m} U\left(t_{m}, t_{m-1}\right) \ldots U\left(t_{l+2}, t_{l-1}\right) Q_{l+1} U\left(t_{t+1}, s\right)
$$

e, portanto,

$$
\begin{aligned}
& \|U(t, s)\|=\left\|U\left(t, t_{m}\right) Q_{m} U\left(t_{m}, t_{m-1}\right) \ldots U\left(t_{l+2}, t_{l+1}\right) Q_{l+1} U\left(t_{l+1}, s\right)\right\| \leq \\
& \leq\left\|U\left(t, t_{m}\right)\right\|\left\|Q_{m}\right\|\left\|U\left(t_{m}, t_{m-1}\right)\right\| \ldots\left\|U\left(t_{l+2}, t_{l+1}\right)\right\|\left\|Q_{l+1}\right\|\left\|U\left(t_{l+1}, s\right)\right\| \\
& =\left(\prod_{t_{1+1} \leq i_{i}<t_{m}}\left\|Q_{i}\right\|\right)\left\|U\left(t, t_{m}\right)\right\|\left\|U\left(t_{m}, t_{m-1}\right)\right\| \ldots\left\|U\left(t_{l+2}, t_{l+1}\right)\right\|\left\|U\left(t_{l+1}, s\right)\right\|=
\end{aligned}
$$

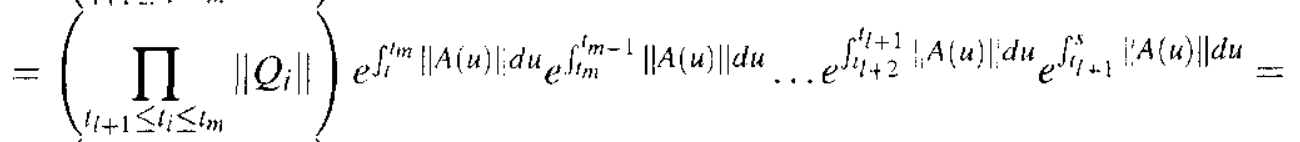

$$
\begin{aligned}
& =\left(\prod_{i_{i+1} \leq i_{i}<l_{m}}\left\|Q_{i}\right\|\right) e^{\int_{t}^{s} \mid \Lambda(u) \| d u}
\end{aligned}
$$

Por outro lado, se $s \geq t$, então existem inteiros $l$ e $m, \operatorname{com} j \leq m \leq l \leq k$, tais que

$$
t_{l} \leq s \leq t_{l+1} \quad \text { e } \quad t_{m} \leq t \leq t_{m+1}
$$

Então

$$
U(t, s)=U\left(t, t_{m+1}\right) Q_{m-1}^{-1} U\left(t_{m+1}, t_{m+2}\right) \ldots U\left(t_{l-1}, t_{l}\right) Q_{l}^{-1} U\left(t_{l}, s\right)
$$


e, portanto,

$$
\begin{aligned}
\|U(t, s)\| & =\left\|U\left(t, t_{m+1}\right) Q_{m+1}^{-1} U\left(t_{m+1}, t_{m+2}\right) \ldots U\left(t_{l-1}, t_{l}\right) Q_{l}^{-1} U\left(t_{l}, s\right)\right\| \leq \\
& \leq\left\|U\left(t, t_{m+1}\right)\right\|\left\|Q_{m+1}^{\cdots 1}\right\|\left\|U\left(t_{m+1}, t_{m+2}\right)\right\| \ldots\left\|U\left(t_{l-1}, t_{l}\right)\right\|\left\|Q_{l}^{-1}\right\|\left\|U\left(t_{l}, s\right)\right\| \|
\end{aligned}
$$

Logo,

$$
\begin{aligned}
& \|U(t, s)\|=\left(\prod_{t_{m+1} \leq t_{i} \leq t_{l}}\left\|Q_{i}^{-1}\right\|\right)\left\|U\left(t, t_{m+1}\right)\right\|\left\|U\left(t_{m+1}, t_{m+2}\right)\right\| \ldots\left\|U\left(t_{l-1}, t_{l}\right)\right\|\left\|U\left(t_{l}, s\right)\right\| \\
& =\left(\prod_{t_{m+1} \leq t_{i} \leq t_{i}}\left\|Q_{i}^{-1}\right\|\right) e^{\int_{t}^{t_{t} m+1}: A(u) \mid d u} e^{\int_{m+1}^{t_{m+2}}\|A(u)\| d u} \ldots e^{\int_{t_{i}}^{t_{i}},\|A(u)\| d u} e^{\int_{t_{i}}^{s}\|A(u)\| d u} \\
& =\left(\prod_{t_{m+1} \leq t_{i} \leq t_{i}}\left\|Q_{i}^{-1}\right\|\right) e^{\int_{i}^{s} \| A(u) \cdot \mid d u} .
\end{aligned}
$$

Donde segue o resultado.

Definição 3.4 Diremos que uma função $A: J \rightarrow L(X)$ é integralmente limitada, se existir $M \geq 0$ tal que

$$
\int_{l}^{t+1}\|A(\tau)\| d \tau \leq M, \quad t \in J
$$

O próximo lema, cuja versão para EDOs pode ser encontrado em [4], Lema 3.1, diz que se a função $A$ em (3.1) for integralmente limitada, então a condição (iii) da Definição 3.2 pode ser omitida.

Lema 3.2 Consideremos a $E D I$ (3.1), (3.2) e suponhamos que $\operatorname{det} Q_{n} \neq 0$, para qualquer $n \in \mathbb{Z}$ tal que $t_{n} \in J$. Se a função $A(t)$ for integralmente limitada, então o item (iii) da Definição 3.2 será uma conseqüência dos itens (i) e (ii) da mesma definição.

Demonstração: Pelo Lema 3.1, a estimativa (3.7) implica que

$$
\|U(t+m, t)\| \leq M_{1} e^{m M}
$$

para todo $m \in \mathbb{Z}$ tal que $t+m \in J$, onde $M_{1}=|j-i| \max \left\{\left\|Q_{k}\right\|,\left\|Q_{k}^{-1}\right\| ; k=i+1,2, \ldots, j\right\}$, com $t \in\left(t_{i}, t_{i+1}\right)$ e $t+m \in\left(t_{j}, t_{j+1}\right)$. 
Consideremos a decomposição $X=X_{1} \oplus X_{2}$ da Definição 3.2. Para cada $t \in J$ fixado, consideremos os vetores $x_{k}(t) \in X_{k}, k=1,2$, tais que

$$
x_{k}(\tau)=U(\tau, t) x_{k}(t), \quad \tau \in J
$$

De (3.4) e (3.5), obtemos as estimativas

$$
\left|x_{1}(t+m)\right| \leq N_{1} e^{-v_{1} m}\left|x_{1}(t)\right|
$$

e

$$
\left|x_{2}(t+m)\right| \geq N_{2}^{-1} e^{v_{2} m}\left|x_{2}(t)\right|
$$

Daí,

$$
\begin{aligned}
\left|x_{1}(t)+x_{2}(t)\right| & =\left\|U(t, t+m) U(t+m, t) x_{1}(t)+U(t, t+m) U(t+m, t) x_{2}(t)\right\| \geq \\
& \geq M_{1} e^{-m M}|| U(t+m, t) x_{1}(t)+U(t+m, t) x_{2}(t) \| \geq \\
& \geq M_{1} e^{-m M}\left(\left\|x_{2}(t+m) \mid !-\right\| x_{1}(t+m) \|\right) \geq \\
& \geq M_{1} e^{-m M}\left(N_{2}{ }^{1} e^{v_{2} m}\left|x_{2}(t)\right|-N_{1} e^{-v_{1} m}\left|x_{1}(t)\right|\right) .
\end{aligned}
$$

e, como $\left|x_{1}(t)\right|=1=\left|x_{2}(t)\right|$, segue que

$$
\left|x_{1}(t)+x_{2}(t)\right| \geq M_{1} e^{-m M}\left(N_{2}^{-1} e^{v_{2} m}-N_{1} e^{-v_{1} m}\right)=\gamma_{m}
$$

donde, por (3.3), temos

$$
S_{n}\left(U(t) X_{1}, U(t) X_{2}\right) \geq \gamma_{m}, \quad \text { para todo } m \in \mathbb{N} \text {. }
$$

Daí, como $\gamma_{m}>0$, para $m$ suficientemente grande, o resultado segue.

Lema 3.3 /[4], Lema 1.1/ Suponhamos que o espaço $X$ seja decomposto como soma direta dos subespaços $X_{1}$ e $X_{2}$, isto é, $X=X_{1} \oplus X_{2}$, e sejam $P_{1}$ e $P_{2}=I-P_{1}$ suas projeçöes correspondentes. Então

$$
\frac{1}{\left\|P_{k}\right\|} \leq S_{n}\left(X_{1}, X_{2}\right) \leq \frac{2}{\left\|P_{k}\right\|}, \quad k=1,2
$$


Demonstração: Escolhamos $\delta>S_{n}\left(X_{1}, X_{2}\right)$ arbitrário. Então existem vetores unitários $x_{k} \in X_{k}$, $k=1,2$, tais que

$$
\left|x_{1}+x_{2}\right|<\delta
$$

Seja $x=x_{1}+x_{2}$. Então, para $k=1,2$, temos $x_{k}=P_{k} x$ e

$$
1=\left|x_{k}\right| \leq\left\|P_{k}|||x|<\right\| P_{k} \| \delta
$$

Assim

$$
\frac{1}{\left\|P_{k}\right\|}<\delta, \quad k=1,2
$$

Como $\delta>S_{n}\left(X_{1}, X_{2}\right)$ é arbitrário, podemos tomar a seqüência $\left\{\delta_{m}\right\}_{m \in \mathbb{N}}$, com $\delta_{m}=S_{n}\left(X_{1}, X_{2}\right)+\frac{1}{m}$, para $m \in \mathbb{N}$. Deste modo,

$$
\frac{1}{\left\|P_{k}\right\|}<\delta_{m}, \quad k=1,2
$$

e, fazendo $m \rightarrow \infty$, temos

$$
\frac{1}{\left\|P_{k}\right\|} \leq S_{n}\left(X_{1}, X_{2}\right), \quad k=1,2, m \in \mathbb{N}
$$

Por outro lado, dado $x \in X$ arbitrário, temos

$$
\begin{aligned}
S_{n}\left(X_{1}, X_{2}\right) & \leq\left|\frac{P_{1} x}{\left|P_{1} x\right|}+\frac{P_{2} x}{\left|P_{2} x\right|}\right| \leq \frac{1}{\left|P_{1} x\right|}\left|P_{1} x+\frac{\left|P_{1} x\right|}{\left|P_{2} x\right|} P_{2} x\right|= \\
& =\frac{1}{\left|P_{1} x\right|}\left|x+\frac{\left|P_{1} x\right|-\left|P_{2} x\right|}{\left|P_{2} x\right|} P_{2} x\right| \leq \frac{1}{\left|P_{1} x\right|}\left\{|x|+\left|\frac{\left|P_{1} x+P_{2} x\right|}{\left|P_{2} x\right|} P_{2} x\right|\right\} \leq 2 \frac{|x|}{\left|P_{1} x\right|} .
\end{aligned}
$$

Portanto

$$
S_{n}\left(X_{1}, X_{2}\right) \leq \inf \left\{2 \frac{|x|}{\left|P_{1} x\right|}: x \in X,|x|=1\right\}=\frac{2}{\left\|P_{1}\right\| \mid} .
$$

Analogamente, temos

$$
S_{n}\left(X_{1}, X_{2}\right) \leq \inf \left\{2 \frac{|x|}{\left|P_{2} x\right|}: x \in X,|x|=1\right\}=\frac{2}{\left\|P_{2}\right\|}
$$

e a prova está completa.

Observação 3.2 Suponhamos que $P_{1}$ e $P_{2}$ sejam projeções mutuamente complementares sobre os 
espaços $X_{1}$ e $X_{2}$, respectivamente como no enunciado do Lema 3.3. Então

$$
P_{k}=U(t) P_{k} U^{\cdot 1}(t), \quad t \in J, k=1,2
$$

Observação 3.3 Segue do Lema 3.3 que a condição (iii) na Definição 3.2 é equivalente à limitaçāo uniforme das projeções $P_{1}$ e $P_{2}=\left(I-P_{1}\right)$, isto é, existe $M$ tal que

$$
\left\|P_{k}\right\| \leq M, \quad k=1,2 .
$$

O próximo lema nos dá condições necessárias e suficientes para que (3.1), (3.2) admita uma dicotomia exponencial. É importante ressaltar que, nos artigos que estudamos, a definição de dicotomia é dada pela condição suficiente deste lema. Por outro lado, em [1], os autores usam a definição com a qual trabalhamos aqui sem, contudo, demonstrar o lema seguinte.

Lema 3.4 [l]], Lema 5.1] Consideremos a EDI (3.1), (3.2) e suponhamos que $\operatorname{det} Q_{n} \neq 0$, para qualquer $n \in \mathbb{Z}$ tal que $t_{n} \in J$. Entāo (3.1), (3.2) terá uma dicotomia exponencial se, e somente se, existirem constantes positivas $K, \alpha$ e uma projeção $P: X \rightarrow X$ tais que

$$
\left\|U(t) P U^{-1}(s)\right\| \leq K e^{-\alpha(t-s)}, \quad s \leq t ; \quad s, t \in J
$$

$e$

$$
\| U(t)(I-P) U^{-1}(s) ! \mid \leq K e^{-\alpha(s-t)}, \quad s \geq t ; \quad s, t \in J .
$$

Demonstração: Suponhamos que a EDI (3.1), (3.2) tenha uma dicotomia exponencial com decomposição $X=X_{1} \oplus X_{2}$.

Seja $P$ a projeção de $X$ sobre $X_{1}$. Então, para $s \leq t$ com $s, t \in J$, temos, por (3.4), que

$$
\begin{aligned}
\left|U(t) P U^{-1}(s) x\right| & =\left|U(t) x_{0}^{1}\right| \leq N_{1} e^{-v_{1}\langle t-s\rangle}\left|U(s) x_{0}^{1}\right|=N_{1} e^{-v_{1}(t-s)}\left|U(s) P U^{-1}(s) x\right|= \\
& =N_{1} e^{-v_{1}(t-s)}|P x| \leq N_{1} e^{-v_{1}(t-s)}|| P|:| x \mid \leq \\
& \leq N_{1} M_{1} e^{-v_{1}(t-s)}|x|,
\end{aligned}
$$


donde

$$
\left\|U(t) P U^{-1}(s)\right\| \leq N_{1} M_{1} e^{-v_{1}(t-s)} .
$$

Analogamente, tomando a projeção $I-P$, para $s \geq t \operatorname{com} s, t \in J,(3.5)$ implica

$$
\begin{aligned}
\left|U(t)(I-P) U^{-1}(s) x\right| & =\left|U(t) x_{0}^{2}\right| \leq N_{2} e^{-v_{2}(s-t)}\left|U(s) x_{0}^{2}\right|= \\
& =N_{2} e^{-v_{2}(s-t)}\left|U(s)(I-P) U^{-1}(s) x\right|=N_{2} e^{-v_{2}(s-t)}|(I-P) x| \leq \\
& \leq N_{2} e^{--v_{2}(s-t)}|| I-P|||x| \leq N_{2} M_{2} e^{-v_{2}(t-s)}|x|
\end{aligned}
$$

e, portanto,

$$
\left\|U(t)(I-P) U^{-1}(s)\right\| \leq N_{2} M_{2} e^{-v_{2}(s-t)}
$$

Finalmente, fazendo $\alpha=\min \left\{v_{1}, v_{2}\right\}$ e $K=\max \left\{N_{1} M_{1}, N_{2} M_{2}\right\}$, segue de (3.10) e (3.11) que

$$
\left\|U(t) P U^{-1}(s)\right\| \leq K e^{-\alpha(t-s)}
$$

e

$$
\left\|U(t)(I-P) U^{-1}(s)\right\| \leq K e^{-\alpha(s-l)} .
$$

Reciprocamente, suponhamos que existam constantes positivas $K$ e $\alpha$ e uma projeção $P: X \rightarrow X$ tais que

$$
\mid{ }_{i}^{\prime} U(t) P U^{1}(s) \| \leq K e^{-\alpha(t-s)}, \quad t \geq s ; t, s \in J
$$

e

$$
\left\|U(t)(I-P) U^{-1}(s)\right\| \leq K e^{-\alpha(s-t)}, \quad t \leq s ; s, t \in J .
$$

Tomemos $X_{1}=P X$ e $X_{2}=(I-P) X$. Logo $X=X_{1} \oplus X_{2}$ e valem

$$
\begin{aligned}
\left|x_{1}(t)\right| & =|U(t) P x(0)|=\left|U(t) P U^{-1}(s) x(s)\right| \leq \\
& \leq|| U(t) P U^{-1}(s) \||x(s)| \leq K e^{-\alpha(t-s)}|x(s)|
\end{aligned}
$$

e

$$
\begin{aligned}
\left|x_{2}(t)\right| & =|U(t)(I-P) x(0)|=\left|U(t)(I-P) U^{1}(s) x(s)\right| \leq \\
& \leq\left\|U(t)(I-P) U^{\mathrm{I}}(s)\right\||x(s)| \leq K e^{-\alpha(t-s)}|x(s)| .
\end{aligned}
$$


Pclas hipóteses, temos

$$
\left\|U(t) P U^{-1}(s)\right\| \leq K e^{-\alpha(t-s)}, \quad s \leq t ; s, t \in J
$$

c

$$
\left\|U(t)(I-P) U^{-1}(s)\right\| \leq K e^{-\alpha(s-t)}, \quad s \geq t ; s, t \in J .
$$

Daí, para $s=t$, segue que

$$
\|P\|=\left\|U(t) P U^{-1}(t)\right\| \leq K, \quad t \in J
$$

e

$$
\|I-P\|=\left\|U(t)(I-P) U^{-1}(t)\right\| \leq K, \quad t \in J .
$$

Então, pela Observação 3.3 , segue que

$$
S_{n}\left(U(t) X_{1}, U(t) x_{2}\right) \geq K=\gamma
$$

Desta forma, a EDI (3.1), (3.2) tem uma dicotomia exponencial e o lema está provado.

Uma característica importante da dicotomia exponencial é a seguinte: se a EDI (3.1), (3.2) tiver uma dicotomia exponencial sobre ambas as semi-retas $\mathbb{R}_{-}$e $\mathbb{R}_{+}$com mesma projeção $P: X \rightarrow X$, constantes $\alpha, K_{1}$ e $\beta, K_{2}$, respectivamente, então a EDI (3.1), (3.2) terá uma dicotomia exponencial sobre $\mathbb{R}$, com a mesma projeção $P$ e constantes que dependerão somente de $\alpha, K_{1}, \beta$ e $K_{2}$.

A seguir, apresentamos alguns exemplos de dicotomia exponencial.

Exemplo 3.1 Consideremos o problema impulsivo (3.1), (3.2), com $A(t)=A, t \in \mathbb{R}_{+}, I_{n}=I$, $n \in \mathbb{N}$, e suponhamos que $A Q=Q A$, onde $Q=I+I=2 I$. Suponhamos que os pontos $\left\{t_{n}\right\}_{n \in \mathbb{N}}$ formem uma progressão aritmética, isto é, $t_{n}=n h, n \in \mathbb{N}, h>0$. Então a EDI (3.1), (3.2) tem a forma

$$
\begin{aligned}
& \frac{d x}{d t}=A x, \quad t \neq n h, n \in \mathbb{N}, \\
& \Delta x\left(t_{n}\right)=2 I x\left(t_{n}\right), \quad n \in \mathbb{N},
\end{aligned}
$$


Mostraremos que a EDI (3.12), (3.13) terá uma dicotomia exponencial, se o espectro do operador $A+h \ln Q$ não interceptar o eixo imaginário. De fato, neste caso o operador de evolução $U(t)$ tem a forma

$$
U(t)=e^{A t} Q^{t / h}
$$

e o espectro do operador $A+h \ln Q$ pode ser escrito como soma direta de duas partes $\sigma_{1}$ e $\sigma_{2}$, onde $\sigma_{1}$ está contido no semi-plano esquerdo e $\sigma_{2}$ está contido no semi-plano direito. Sendo

$$
X=X_{1} \oplus X_{2}
$$

a decomposição correspondente do espaço X como uma soma direta e sendo $P$ a projeçāo correspondente a $X_{1}$, não é difícil verificar que

$$
\left\|U(t) P U^{\cdots 1}(s)\right\| \leq K_{1} e^{\mu_{1}(t-s)}, \quad 0 \leq s<t<\infty,
$$

$e$

$$
\left\|U(t)(I-P) U^{-1}(s)\right\| \leq K_{2} e^{\mu_{2}(t-s)}, \quad 0 \leq t<s<\infty
$$

onde $\mu_{1}$ é um número arbitrário pertencente ao intervalo (sup $\left.\sigma_{1}, 0\right)$, $\mu_{2}$ é um número arbitrário pertencente ao intervalo $\left(\sup \sigma_{2}, 0\right)$ e $K_{1}, K_{2}$ são constantes positivas.

Exemplo 3.2 Consideremos a EDI (3.1), (3.2), onde $A(t), 0 \leq t<\infty e Q_{n}=I+I_{n}, n \in \mathbb{N}$, comutam entre si, isto é,

$$
A(t) A(s)=A(s) A(t), \quad 0 \leq t, s<\infty,
$$

$$
A(t) I_{n}=I_{n} A(t), \quad 0 \leq t<\infty, n \in \mathbb{N},
$$

Neste caso, o operador de evolução U(t) tem a forma

$$
U(t)=e^{\int_{0}^{t} A(s) d s}\left(I+I_{n(t)}\right) \ldots\left(I+I_{1}\right)
$$

onde $n(t)=\min \left\{n: t<t_{n}\right\}$. Entāo a $E D I(3.1)$, (3.2) terá uma dicotomia exponencial, se existir uma projeção $P$ que comute com $A(t), 0 \leq t<\infty$, e os operadores $I_{n}, n \in \mathbb{N}$, sejam tais que para 
algumas constantes positivas $K$ e $\alpha$, as seguintes desigualdades sejam válidas:

$$
\begin{gathered}
\left\|e^{\int_{\tau}^{t} A(s) d s}\left(I+I_{n(t)}\right) \ldots\left(I+I_{n(\tau)}\right) P\right\| \leq K e^{-\alpha(t-\tau)}, \quad 0 \leq \tau<t<\infty, \\
\left\|e^{\int_{\tau}^{t} A(s) d s}\left(I+I_{n(t)}\right)^{-1} \ldots\left(I+I_{n(\tau)}\right)^{-1}(I-P)\right\| \leq K e^{-\alpha(\tau-t)}, \quad 0 \leq t<\tau<\infty .
\end{gathered}
$$

Exemplo 3.3 Consideremos a EDI (3.1), (3.2), onde $A(t), 0 \leq t<+\infty$, e as seqüências $\left\{t_{n}\right\}_{n \in \mathbb{N}}$ $e\left\{I_{n}\right\}_{n \in \mathbb{N}}$ são $\omega$-periódicas, isto é, existem $\omega>0$ e um inteiro positivo l tais que as seguintes igualdades são válidas:

$$
\begin{array}{cc}
A(t+\omega)=A(t), & 0 \leq t<\infty, \\
t_{n}+\omega=t_{n+l,} & n \in \mathbb{N}, \\
I_{n+j(\omega)}=I_{n}, & n \in \mathbb{N} .
\end{array}
$$

Então o operador de evolução $U(t)$ satisfaz.

$$
U(t+\omega)=U(t) U(\omega)
$$

A EDI (3.1), (3.2) terá uma dicotomia exponencial, se o espectro do operador $U(\omega)$ não interceptar o círculo unitário. De fato, neste caso, o espaço X pode ser escrito como uma soma direta

$$
X=X_{1} \oplus X_{2}
$$

onde $X_{1}$ e $X_{2}$ são invariantes com respeito a $U(\omega)$.

$O$ espectro da restriça a de $U(\omega)$ a $X_{1}$ pertence ao interior do círculo unitário e o espectro da restrição de $U(\omega)$ a $X_{2}$ pertence ao exterior do círculo unitário. Se $P$ for a projeção correspondente à decomposição $X=X_{1} \oplus X_{2}, \operatorname{com} X_{1}=P X$, entāo a $E D I$ (3.1), (3.2) terá uma dicotomia exponencial com a projeção $P$ mencionada.

Agora, vamos definir dicotomia ordinária para a EDI (3.1), (3.2). 
Definição 3.5 Consideremos a $E D I$ (3.1), (3.2) e suponhamos que $\operatorname{det} Q_{n} \neq 0$, para todo $n \in \mathbb{Z}$ tal que $t_{n} \in J$. Diremos que (3.1), (3.2) tem uma dicotomia ordinária sobre J, se existirem uma constante positiva $K$ e uma projeção $P: X \rightarrow X$ tais que

$$
\left\|U(t) P U^{-1}(s)\right\| \leq K, \quad s, t \in J
$$

$$
\left\|U(t)(I-P) U^{-1}(s)\right\| \leq K, \quad s, t \in J
$$

Vale observarmos que, se a EDI (3.1), (3.2) tiver uma dicotomia exponencial ou uma dicotomia ordinária sobre um intervalo $\left[t_{0}, \infty\right), t_{0} \in \mathbb{R}_{+}$, então ela também terá, respectivamente, uma dicotomia exponencial ou uma dicotomia ordinária sobre a semi-reta $\mathbb{R}_{+}$, com a mesma projeção $P: X \rightarrow X$ e o mesmo expoente $\alpha$, no caso da dicotomia exponencial. De fato. Podemos escolher

$$
M=M_{1} e^{\int_{0}^{t}\|\| A(u) ! d u}
$$

onde $M_{1}=\left|i\left(0, t_{0}\right)\right| \max \left\{\left\|Q_{j}||,\right\| Q_{j}^{-1} \|: t_{j} \in\left(0, t_{0}\right)\right\}$. Deste modo, pelo Lema 3.1, temos

$$
\|U(t, s)\|_{i} \leq M, \quad 0 \leq s, t \leq t_{0} .
$$

Suponhamos que $0 \leq s \leq t \leq t_{0}$. Então,

$$
\left\|U(t) P U^{-1}(s)\right\| \leq M\left|x(t) P U^{-1}\left(t_{0}\right)\right| \leq M K e^{-\alpha\left(t-t_{0}\right)} \leq M K e^{\alpha t_{0}} e^{-\alpha(t-s)} .
$$

Agora, se supusermos que $0 \leq s \leq t_{0} \leq t<\infty$, teremos

$$
i\left|U(t) P U^{-1}(s)\right|\left|\leq M^{2}\right| U\left(t_{0}\right) P U^{-1}\left(t_{0}\right) \mid \leq M^{2} K \leq M^{2} K e^{\alpha t_{0}} e^{-\alpha(t-s)} .
$$

Portanto

$$
{ }_{i}^{1} U(t) P U^{-1}(s) \| \leq \tilde{K} e^{-\alpha(t-s)}, \quad 0 \leq s \leq t<\infty,
$$

onde $\vec{K}=M^{2} K e^{\alpha t_{0}}$. 
Utilizando um argumento análogo para $I-P$, chegaremos em

$$
\left\|U(t)(I-P) U^{-1}(s)\right\| \leq \tilde{K} e^{-\alpha(t-s)}, \quad 0 \leq s \leq t<\infty
$$

c, deste modo, nossa afirmação está provada.

No restante desta seção, assumiremos que $\operatorname{det} Q_{n} \neq 0$, para todo $n$ tal que $t_{n} \in J$ na EDI (3.1), (3.2). Isto será necessário para definirmos dicotomia espectral. Além disso, esta hipótese também será utilizada implicitamente na definição do inverso do operador característico da EDI (3.1), (3.2) que definiremos abaixo, bem como no Teorema 3.1.

Seja $\mathscr{H}$ o espaço de Banach

$$
\left\{v: \mathbb{R} \rightarrow X: \sup _{t \in \mathbb{R}}|v(t)|<\infty\right\}
$$

munido da norma $|! v|=\sup _{t \in \mathbb{R}}|v(t)|$.

Definição 3.6 Chamamos de operador característico da $E D I(3.1),(3.2)$, para $J=\mathbb{R}$, o operador $T: \mathscr{H} \rightarrow \mathscr{H}$ dado por

$$
(T v)(t)=U(t, t-1) v(t-1), \quad t \in \mathbb{R}
$$

para todo $v \in \mathscr{H}$. Então $T^{-1}: \mathscr{H} \rightarrow \mathscr{H}$ é definido por

$$
\left(T^{-1} v\right)(t)=U(t, t+1) v(t+1), \quad t \in \mathbb{R}
$$

Observemos que, para o inverso do operador característico da EDI (3.1), (3.2) estar definido, é necessário que $\operatorname{det} Q_{n} \neq 0$, para qualquer $n \in \mathbb{Z}$, como estamos supondo.

Definição 3.7 Diremos que a $E D I(3.1),(3.2)\left(\right.$ com $J=\mathbb{R}$ ou $\left.J=\mathbb{R}_{+}\right)$tem uma dicotomia espectral se $\sigma(T) \cap S^{1}-\emptyset$, onde $T$ é o operador característico da $E D I$ (3.1), (3.2), $\sigma(T)$ é o espectro da complexificaçăa de $T$ e $S^{1}$ é o círculo unitário no plano complexo.

A versão original do próximo tcorema se encontra em [1], Teorema 5.1, onde os autores não consideram a hipótese da invertibilidade das matrizes $Q_{n}$ e que $g(X)<+\infty$ e que $g^{\prime}(X)>-\infty$. 
Isto implica que o operador de evolução, $U(t, s)$, da EDI (3.1), (3.2) está definido somente para $s \leq t$. Entretanto, no enunciado e no decorrer da demonstração, eles utilizam o inverso do operador característico que é definido por

$$
\left(T^{-1} v\right)(t)=U(t, t+1) v(t+1), \quad t \in \mathbb{R}
$$

o que mostra que a hipótese de que as matrizes $Q_{n}$ sejam invertíveis é necessária. Além disso, eles utilizam o fato dos expoentes geral superior e geral inferior das soluções da EDI (3.1), (3.2) serem limitados. O objetivo do próximo teorema é relacionar os expoentes geral superior e geral inferior das soluções da EDI (3.1), (3.2) com o operador característico $T$ desta equação.

Teorema 3.1 [[1], Teorema 5.1] Consideremos a EDI (3.1), (3.2), com $J=\mathbb{R}$ (ou $J=R_{+}$), e suponhamos que $\operatorname{det} Q_{n} \neq 0$, para qualquer $n \in \mathbb{Z}$ (respectivamente, $n \in \mathbb{N}$ ). Suponhamos, também, que $g(X)<+\infty$ e que $g^{\prime}(X)>-\infty$. Entāo

$$
g(X)=\ln r_{\sigma}(T)
$$

$$
g^{\prime}(X)=-\ln r_{\sigma}\left(T^{-1}\right)
$$

onde T é o operador característico de (3.1), (3.2) e $r_{\sigma}(T)$ é o raio espectral da complexificação de $T$

Demonstração: Primeiramente, mostraremos (3.21). Provemos, inicialmente, que

$$
g(X) \geq \ln r_{\sigma}(T) .
$$

Suponhamos que exista $q \in \mathbb{R}$ tal que

$$
|U(t, s) x| \leq N_{y} e^{q(t-s)}|x|, \quad \text { para } s \leq t .
$$

Notemos que tal número $q$ existe pela limitação do expoente geral superior, $g(X)$. Então

$$
\left|\left(T^{k} v\right)(t)\right|=|U(t, t-k) v(t-k)| \leq N_{q} e^{q k}|v(t-k)|,
$$


para $k \in \mathbb{N}, v \in \mathscr{H}, t \in \mathbb{R}$. Portanto

$$
\left\|T^{k} v\right\| \leq N_{q} e^{q k}\|v\|
$$

para todo $v \in \mathscr{H}$. Pelo Teorema do Raio Espectral (Teorema 1.5), segue que

$$
r_{\sigma}(T)=\lim _{k \rightarrow \infty}\left\|T^{k}\right\|^{\frac{1}{k}} \leq \lim _{k \rightarrow, \infty}\left\|N_{q} e^{q k}\right\|^{\frac{1}{k}}=e^{q}
$$

Isto prova (3.23)

Suponhamos, agora, que exista um número real positivo $p$ tal que $p>r_{\sigma}(T)$. Novamente, pelo Teorema do Raio Espectral (Teorema 1.5) temos que, para $k_{0}$ suficientemente grande, vale

$$
\|\left. T^{k_{0}}\right|_{i} ^{\frac{1}{k_{0}}} \leq p
$$

Seja $x \in X$ e definamos $v_{x} \in \mathscr{H}$ por

$$
v_{x}(t)= \begin{cases}0, & \text { para } t \neq s \\ x, & \text { para } t=s .\end{cases}
$$

De (3.24) segue que

$$
\left\|T^{k_{0}} v_{x}\right\| \leq p^{k_{0}}\left\|v_{x}\right\| .
$$

Pelas definições do operador característico $T$ e de $v_{x}$, segue que

$$
\begin{aligned}
\sup _{t \in \mathbb{R}}\left|T^{k_{0}} v_{x}(t)\right| & =\sup _{t \in \mathbb{R}}\left|U\left(t, t-k_{0}\right) v_{x}\left(t-k_{0}\right)\right|=\left|U\left(t, t-k_{0}\right) x\right|= \\
& =\left\|T^{k_{0}} v_{x}\right\| \leq p^{k_{0}}|| v_{x} \|=p^{k_{0}}|x| .
\end{aligned}
$$

Daí,

$$
|U(t, s) x| \leq p^{(t-s)}|x|
$$

c, pela limitação da expoente geral superior, chegamos à conclusão que existe um número positivo $N_{p}$ lal que

$$
|U(t, s) x| \leq N_{p} p^{(t-s)}|x|
$$


para todo $s \leq t$ e todo $x \in X$. Isto implica que

$$
\ln p \geq g(X)
$$

o que prova $(3.21)$

Analogamente, prova-se (3.22).

\subsection{Redutibilidade}

Consideremos a EDI

$$
\begin{gathered}
\frac{d x}{d t}=B(t) x, \quad t \neq t_{n}, \\
x\left(t_{n}^{\cdot}\right)=x\left(t_{n}\right)+J_{n}\left(x\left(t_{n}\right)\right):=R_{n}\left(x\left(t_{n}\right)\right),
\end{gathered}
$$

onde

- $B: J \subset \mathbb{R} \rightarrow L(X)$ é uma função contínua por partes, com descontinuidade de primeira espécie em $t=t_{n}$, contínua à esquerda em $t=t_{n}$, para qualquer $n \in \mathbb{Z}$ tal que $t_{n} \in J$;

- $J_{n}: X \rightarrow X$ é um operador contínuo, para qualquer $n \in \mathbb{Z}$ tal que $t_{n} \in J$.

Definição 3.8 Diremos que a EDI (3.25), (3.26) é cinematicamente equivalente (ou cinematicamente similar) à $E D I$ (3.1), (3.2), se existir uma função a valores matriciais $S: J \subset \mathbb{R} \rightarrow L(X)$ satisfazendo as seguintes propriedades:

(i) $S(t)$ é limitada e diferenciável em $t \neq t_{n}$, para todo $n \in \mathbb{Z}$ tal que $t_{n} \in J$;

(ii) $S(t)$ tem descontinuidade de primeira espécie em $t=t_{n}$ é é contínua à esquerda em $t=t_{n}$, para todo $n \in \mathbb{Z}$, tal que $t_{n} \in J$

(iii) $S(t)$ é invertível com $S^{-1}(t)$ limitada;

(iv) Se $x(t)$ for uma soluçāo de (3.1), (3.2), então $S(t) x(t)$ será uma solução de (3.25), (3.26). 
Segue diretamente da definição que a equivalência cinemática é uma relação de equivalência.

Podemos facilmente verificar que uma condição necessária e suficiente para a EDI (3.1), (3.2) ser cinematicamente equivalente à EDI (3.25), (3.26) é a existência da função a valores matriciais $S(t), t \in J$, satisfazendo as propriedades $(i),(i i)$, (iii) da Definição 3.8 tal que pelo menos uma das três condições a seguir seja satisfeita

(i) para $t \in J$, com $t \neq t_{n}$, temos

$$
B=S A S^{-1}+S^{\prime} S^{-1}
$$

$\mathrm{e}$

$$
S\left(t_{n}^{+}\right) Q_{n}=R_{n} S\left(t_{n}\right)
$$

para todo $n \in \mathbb{Z}$ tal que $t_{n} \in J$.

(ii) para $t \in J$, temos

$$
S(t)=V(t) U^{-1}(t)
$$

onde $U(t)$ e $V(t)$ são os operadores de evolução de (3.1), (3.2) e de (3.25), (3.26) respectivamente.

(iii) $S$ é solução da seguinte equação

$$
\begin{gathered}
\frac{d S}{d t}=B(t) S-S A(t), \quad t \in J, t \neq t_{n}, \\
S\left(t_{n}^{+}\right)=R_{n} S\left(t_{n}\right) Q_{n}^{-1},
\end{gathered}
$$

para todo $n \in \mathbb{Z}$ tal que $t_{n} \in J$.

A seguir temos uma observação importante.

Observação 3.4 A equivalência cinemática preserva as dicotomias exponencial, ordinária e espectral.

O próximo lema será importante no seguinte sentido: sempre que tivermos projeções $P$ e $I-P$ não necessariamente hermitianas, poderemos encontrar uma outra decomposição do espaço $X$ com 
novas projeções $P_{1}$ e $I-P_{1}$ que sejam hermitianas.

Lema 3.5 [[1], Lema 6.1] Suponhamos que $X$ possa ser escrito como soma direta de dois subespaços $X_{1}$ e $X_{2}$ com projeções $P$ e $I-P$, isto é, $X=X_{1} \oplus X_{2} \operatorname{com} X_{1}=P X$ e $X_{2}=(I-P) X$. Então $X$ poderá ser representado na forma $X=X_{1} \oplus Y_{2}$, onde as projeçôes $P_{1}: X \rightarrow X_{1} e\left(I-P_{1}\right): X \rightarrow Y_{2}$ são operadores hermitianos.

Demonstração: Seja $Y_{1}$ um complemento ortogonal de $X_{1}$ em $X$. Então a projeção $P_{1}: X \rightarrow X_{1}$ com Ker $P_{1}=Y_{1}$ é hermitiana. A projeção $I-P_{1}$ também é hermitiana e $X$ pode ser escrito como soma direta de $X_{1}$ e $Y_{2}=\left(I-P_{1}\right) X$.

O próximo teorema nos diz que, sempre que a EDI (3.1), (3.2) tiver uma dicotomia ordinária cujas projeções $P$ e $I-P$ sejam hermitianas, poderemos encontrar uma outra EDI cinematicamente equivalente à primeira, com algumas propriedades interessantes.

Teorema 3.2 /II/, Teorema 6.1J Suponhamos que a EDI (3.1), (3.2) tenha uma dicotomia ordinária. Suponhamos, ainda, que as projeções $P$ e I-P da Definição 3.5 sejam projeções hermitianas. Então existirá uma EDI que será cinematicamente equivalente à EDI (3.1), (3.2), digamos a EDI (3.25), (3.26), com B(t) e $J_{n}$ comutando com P e I-P. Além disso,

$$
\left\|B(t)+B^{*}(t)\right\| \leq\left\|A(t)+A^{*}(t)\right\|, \quad t \in J
$$

Demonstração: Seja

$$
R^{2}(t)=P_{1} U^{*}(t) U(t) P_{1}+P_{2} U^{*}(t) U(t) P_{2}
$$

Notemos que o operador $R^{2}(t)$ é uniformemente positivo e hermitiano para todo $t \in J$. Suponhamos que $R(t)$ seja uma raiz quadrada positiva de $R^{2}(t)$. Pela Fórmula de Riesz (veja Seção 1.2.2), segue que

$$
R(t)=-\frac{1}{2 \pi i} \oint_{\Gamma_{t}} \sqrt{\lambda}\left[R^{2}(t)-\lambda I\right]^{-1} d \lambda,
$$

onde $\Gamma_{t}$ é uma fronteira suave dependendo continuamente de $t$, cercando o espectro de $R^{2}(t)$ no semi-plano $\operatorname{Re} \lambda>0$ e orientada no sentido anti-horário. Por $\sqrt{\lambda}$ denotamos o valor principal da 
raiz de $\lambda$. Como $R^{2}(t)$ é hermitiano, segue que $R(t)$ também é hermitiano. Pelo fato de $R^{2}(t)$ comutar com $P_{k}, k=1,2$, segue de (3.31) que $R(t)$ comuta com $P_{k}, k=1,2$, como queríamos.

Consideremos a função a valores matriciais $S(t)=R(t) U^{-1}(t), t \in J$. Por (3.31), as funções a valores matriciais $S(t) \mathrm{e} S^{-1}(t)$ têm descontinuidade de primeira espécie em $t=t_{n}$, são diferenciáveis $\mathrm{cm} t \neq t_{n} \mathrm{e}$ são contínuas à esquerda em $t=t_{n}$.

Provemos que $S(t)$ é limitado para cada $t \in J$. Da igualdade

$$
S^{*} S=\left(U^{-1}\right)^{*} R^{2} U^{-1}=\left(U P_{1} U^{-1}\right)^{*}\left(U P_{1} U^{-1}\right)+\left(U P_{2} U^{-1}\right)^{*}\left(U P_{2} U^{-1}\right)
$$

chegamos à seguinte estimativa 


$$
\|S\|^{2} \leq\left\|U P_{1} U^{-1}\right\|+\left\|U P_{2} U^{-1}\right\|<M^{2}+M^{2}<\infty
$$

Daí, como $|S(t)\|\leq\| S \|||t|$, segue que $S(t)$ é limitado para cada $t \in J$.

Provemos, agora, que $S^{-1}=U R^{-1}$ é limitado. De fato. Temos

$$
\begin{aligned}
P_{1}\left(S^{-1}\right)^{*} S^{-1} P_{1}+P_{2}\left(S^{-1}\right)^{*} S^{-1} P_{2} & =P_{1} R^{-1} U^{*} U R^{-1} P_{1}+P_{2} R^{-1} U^{*} U R^{-1} P_{2}= \\
& =R^{-1}\left(P_{1} U^{*} U P_{1}+P_{2} U^{*} U P_{2}\right) R^{-1}=I
\end{aligned}
$$

Então, tomando $z \in X$ arbitrário, temos

$$
\begin{aligned}
\left|S^{-1} z\right|^{2} & =\left|S^{-1} P_{1} z+S^{-1} P_{2} z\right|^{2} \leq 2\left|S^{-1} P_{1} z\right|^{2}+2\left|S^{-1} P_{2} z\right|^{2}= \\
& =2\left(S^{-1} P_{1} z, S^{-1} P_{1} z\right)+2\left(S^{-1} P_{2} z, S^{-1} P_{2} z\right)= \\
& =2\left(P_{1}\left(S^{-1}\right)^{*} S^{-1} P_{1} z, z\right)+2\left(P_{2}\left(S^{-1}\right)^{*} S^{-1} P_{2} z, z\right)= \\
& =2(z, z)=2|z|^{2} .
\end{aligned}
$$

Daí, como $\left\|S^{-1}(t)\right\| \leq\left\|S^{-1}\right\||t|$, segue que $S^{-1}(t)$ é limitado para cada $t \in J$.

Resta mostrarmos que se $x(t)$ for uma solução da EDI (3.1), (3.2), então $S(t) x(t)$ será uma solução da EDI (3.25), (3.26). Por (3.27), isto é equivalente a mostrarmos que

$$
B=S A S^{-1}+S^{\prime} S^{-1}
$$

Como $\frac{d U^{-1}}{d t}=-U^{-1} \frac{d U}{d t} U^{-1}=-U^{-1} A$, então

$$
B=R U^{-1} A U R^{-1}+\left(\frac{d R}{d t} U^{-1}-R U^{-1} A\right) U R^{-1}=\frac{d R}{d t} R^{-1}
$$

Devido a (3.28), temos

$$
J_{n}=S\left(t_{n}^{+}\right) Q_{n} S^{-1}\left(t_{n}\right)=R\left(t_{n}^{+}\right) U^{-1}\left(t_{n}\right) Q_{n}^{-1} Q_{n} U\left(t_{n}\right) R^{-1}\left(t_{n}\right)=R\left(t_{n}^{+}\right) R^{-1}\left(t_{n}\right)
$$

Portanto a EDI (3.1), (3.2) é cinematicamente equivalente à EDI (3.25), (3.26), com $B(t)$ e $J_{n}$ comutando com $P_{k}, k=1,2$. 
Agora, provemos (3.29). Como o operador de evolução de (3.25), (3.26) é

$$
V(t)=S(t) U(t)=R(t)
$$

então, diferenciando ambos os lados de (3.30), obtemos

$$
R \frac{d R}{d t}+\frac{d R}{d t} R=P_{1} U^{*}\left(A^{*}+A\right) U P_{1}+P_{2} U^{*}\left(A^{*}+A\right) U P_{2}
$$

Então, para todo $z \in X$, vale

$$
\begin{gathered}
\left(\left(R(t) \frac{d R}{d t}(t)+\frac{d R}{d t}(t) R(t)\right) z, z\right)= \\
=\left(\left(A^{*}(t)+A(t)\right) U(t) P_{1} z, U(t) P_{1} z\right)+\left(\left(A^{*}(t)+A(t)\right) U(t) P_{2} z, U(t) P_{2} z\right) .
\end{gathered}
$$

Seja $\beta(t)=\left\|A(t)+A^{*}(t)\right\|, t \in J$. De (3.33), temos a estimativa

$$
\begin{aligned}
\left(\left(R(t) \frac{d R}{d t}(t)+\frac{d R}{d t}(t) R(t)\right) z, z\right) & \leq \beta(t)\left\{\left(U(t) P_{1} z, U(t) P_{1} z\right)+\left(U(t) P_{2} z, U(t) P_{2} z\right)\right\}= \\
& =\beta(t)\left(R^{2}(t) z, z\right)=\beta(t)\left\{\left.R(t) z\right|^{2}\right.
\end{aligned}
$$

para $z \in X$ e $t \in J$. Disto, de $R(t) \frac{d R}{d t}(t)+\frac{d R}{d t}(t) R(t)=R(t)\left(B(t)+B^{*}(t)\right) R(t)$ e devido a (3.32), chegamos a

$$
\left.\left(R(t)\left(B(t)+B^{*}(t)\right) R(t)\right) z, z\right) \leq \beta(t)|R(t) z|^{2}
$$

ou seja,

$$
\left.\left(\left(B(t)+B^{*}(t)\right) R(t)\right) z, R(t) z\right) \leq \beta(t)|R(t) z|^{2} .
$$

Então, fazendo $v=R(t) z$, obtemos

$$
\left(\left(B(t)+B^{*}(t)\right) v, v\right) \leq \beta(t)|v|^{2}
$$

Em outras palavras,

$$
\left\|B(t)+B^{*}(t)\right\| \leq \beta(t)=\left\|A(t)+A^{*}(t)\right\|,
$$

para $t \in J$. 


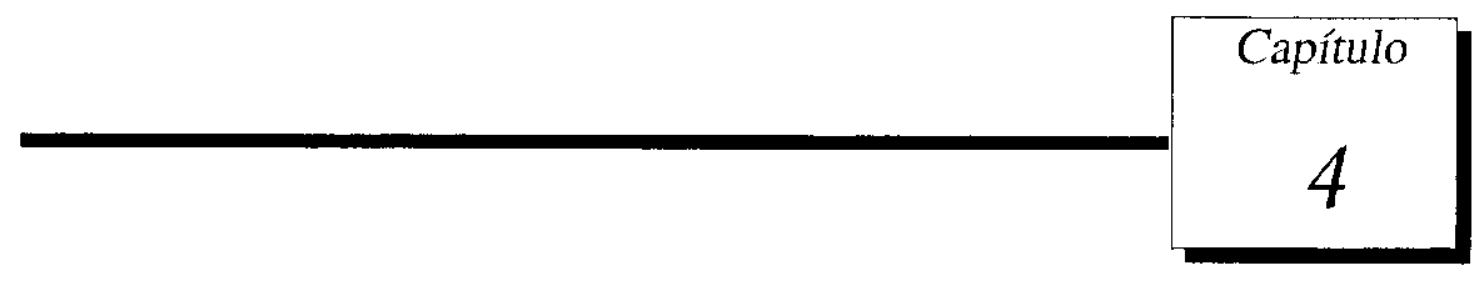

Crescimento Limitado e Dicotomias

Neste capítulo, apresentamos vários resuliados sobre crescimento limitado e dicotomia para a EDI linear homogênea que estamos estudando. Começamos por apresentar condições necessárias e suficientes para o crescimento limitado. Em seguida, discutimos uma relação entre dicotomia exponencial e crescimento limitado para a EDI.

É importante observarmos que nos artigos [2] e [6], os autores não consideram, explicitamente, a hipótese da invertibilidade das matrizes $Q_{n}$, para $n \in \mathbb{Z}$ tal que $t_{n} \in J$. Isto implica que o operador de evolução, $U(t, s)$, da EDI (3.1), (3.2) é definido somente para $s \leq t$. Entretanto, no decorrer destes mesmos artigos, é utilizado o operador de evolução, $U(t, s)$, com $s \geq t$, e para este operador estar definido, é necessária a hipótese de que as matrizes $Q_{n}$ sejam invertíveis. Aqui, nós apresentamos vários resultado com a condição de inivertibilidade.

\subsection{Definições e Propriedades}

Nesta primeira seção, nós definimos crescimento limitado e, em seguida, apresentamos uma condição necessária e suficiente para que a EDI (3.1), (3.2) tenha crescimento limitado. Depois disto, apresentamos algumas condições que implicam nesta condição suficiente provando, assim, que a EDI tem crescimento limitado.

Definição 4.1 Diremos que a EDI (3.1), (3.2) tem crescimento limitado sobre J se, para algum 
$h>0$, existir uma constante $C>1$ tal que toda solução $x(t)$ da EDI (3.1), (3.2) satisfaz

$$
|x(t)| \leq C|x(s)|
$$

para $s, t \in J$, com $t \in[s, s+h] \cap J$.

Podemos observar que, tomando $t_{0} \in \mathbb{R}_{-}$, se a EDI (3.1), (3.2) tiver crescimento limitado sobre o intervalo

$J=\left[t_{0}, \infty\right)$, então ela terá crescimento limitado sobre a semi-reta $\mathbb{R}_{+}$. Isto decorre dos fatos das soluções $x(t)$ da EDI (3.1), (3.2) serem contínuas por partes, com descontinuidade de primeira espécie em $t=t_{n}$ e contínuas à esquerda em $t=t_{n}$, para qualquer $n \in \mathbb{Z}$, e de $\left[0, t_{0}\right]$ ser um intervalo compacto. Deste modo, se a EDI tiver crescimento limitado com $|x(t)| \leq C|x(s)|$ como em (4.1), então poderemos definir

$$
\eta=\frac{\max \left\{|x(t)|: t \in\left[0, t_{0}\right]\right\}}{\min \left\{|x(t)|: t \in\left[0, t_{0}\right]\right\}}
$$

e teremos

$$
|x(t)| \leq C_{1}|x(s)|
$$

para $s, t \in \mathbb{R}$ e $t \in[h, s+h]$, onde $C_{1}=C \max \{1, \eta\}$.

Do mesmo modo, se a EDI (3.1), (3.2) tiver crescimento limitado sobre o intervalo $J=\left(-\infty, t_{0}\right]$, então ela terá crescimento limitado sobre a semi-reta $\mathbb{R}_{-}$.

O próximo teorema nos dá uma condição necessária e suficiente para que a EDI (3.1), (3.2) tenha crescimento limitado. O aspecto interessante de tal teorema é que a condição se dá sobre o operador de Cauchy da FDI. Além disso, a partir daqui, utilizaremos este resultado na maior parte dos teoremas para concluirmos que (3.1), (3.2) tem crescimento limitado.

Teorema 4.1 [/6], Teorema 2.I/ Consideremos a EDI (3.1), (3.2) e suponhamos que $\operatorname{det} Q_{n} \neq 0$, para todo $n \subset \mathbb{Z}$ tal que $t_{n} \in J$. Então a $E D I$ (3.1), (3.2) terá crescimento limitado se, e somente se, existirem constantes reais $K$ e $\alpha$ tais que

$$
\|U(t, s)\| \leq K e^{\alpha \mid t^{r-s \mid}}, \quad s, t \in J
$$


Demonstração: Seja $x(t)$ uma solução da EDI (3.1), (3.2). Suponhamos que (4.2) ocorra para alguns valores de $K$ e $\alpha$. Seja $h>0$ fixado e sejam $s, t \in J$ tais que $t \in[s, s+h] \cap J$. Então, fazendo $C=K e^{\alpha h}$, temos

$$
|x(t)| \leq\left|{ }_{i} U(t, s)\right||| x(s)\left|\leq K e^{\alpha(t-s)}\right| x(s)\left|\leq K e^{\alpha h}\right| x(s)|=C| x(s) \mid .
$$

Reciprocamente, suponhamos que a EDI (3.1), (3.2) tenha crescimento limitado e que $h$ seja como na Definição 4.1. Seja $s \leq t$. Então $t \in[s+k h, s+(k+1) h]$ para algum $k \in \mathbb{Z}$. Portanto, temos

$$
|x(t)| \leq C|x(s+k h)| \leq \ldots \leq C^{k+1}|x(s)| .
$$

Fazendo $K=C$ e $\alpha=h^{-1} \ln C$, temos $C^{k}=e^{\alpha k h} \leq e^{\alpha(t-s)}$. Logo

$$
|x(t)| \leq K e^{\alpha(t s)}|x(s)|, \quad s \leq t
$$

E. como $x(t)=U(t, s) x(s)$, temos

$$
\|U(t, s)\|_{i} \leq K e^{\alpha(t-s)}=K e^{\alpha_{i}(-s)}, \quad s \leq t .
$$

Analogamente provamos a desigualdade acima para $s \geq t$, donde segue o resultado.

No teorema que acabamos de provar, a parte em que ocorrem os impulsos não é explícita.

Originalmente, o resultado a seguir pode ser encontrado em [6]. Aqui, em vez de assumirmos a condição de $A$ ser limitada, consideramos a condição mais fraca de $A$ ser integralmente limitada. Neste teorema, damos condiçōes suficientes sobre $A(t)$, sobre os operadores de impulso, $Q_{n}$, com $n \in \mathbb{Z}$ tal que $t_{n} \in J$, e sobre a sequiência de tempos de impulso $\left\{t_{n}\right\}_{n \in \mathbb{N}} \subset J$, a fim de obtermos (4.2) e, deste modo, conseguimos que a EDI (3.1), (3.2) tenha crescimento limitado.

Teorema 4.2 [[6], Teorema 2.2] Consideremos a EDI (3.1), (3.2) e suponhamos que

(i) A(t) seja integralmente limitada sobre J;

(ii) Exista uma constante $K>0$ tal que, para quaisquer inteiros positivos $i$ e $j$, com $i<j$, 
tenhamos

$$
\left\|Q_{i}\right\|\left\|Q_{i+1}\right\| \ldots\left\|Q_{j}\right\| \leq K e^{j-i+1}
$$

(iii) Exista $h>0$ tal que $h<t_{n+1}-t_{n}$, para todo $n \in \mathbb{Z}$ tal que $t_{n} \in J$,

(iv) $\operatorname{det} Q_{n} \neq 0$, para $n \in \mathbb{Z}$ tal que $t_{n} \in J$.

Então

$$
\|U(t, s)\| \leq K_{1} e^{\alpha|t-s|}, \quad s, t \in \mathbb{R}
$$

onde $K_{1}=$ Ke e $\alpha=\delta^{-1} \ln K_{1}+M$.

Demonstração: Suponhamos que $A(t)$ seja integralmente limitada sobre $J$. Logo, existirá uma constante $M>0$ tal que

$$
\int_{i}^{t+1}\|A(s)\| d s \leq M, \quad t \in J
$$

Sejam $s, t \in J, \operatorname{com} s \leq t$. Então existirão inteiros positivos $i$ e $k, \operatorname{com} i \leq k$, tais que $s \in\left(t_{i}, t_{i+1}\right]$ e $t \in\left(t_{k}, t_{k+1}\right\}$. Assim

$$
\|U(t, s)\| \leq\left\|U\left(t, t_{k}\right) !\right\| U\left(t_{k}, t_{k-1}\right)\|\ldots\| U\left(t_{i+1}, s\right)\|\| Q_{k}\|\| Q_{k-1}\|\ldots\| Q_{i+1} \|
$$

Seja $\xi \in X$. Escrevemos

$$
U\left(t, t_{k}\right) \xi=x\left(t ; t_{k}, \xi\right)=\xi+\int_{t_{k}}^{t} A(s) x\left(s ; t_{k}, \xi\right) d s
$$

Passando a norma na igualdade acima e usando o Lema 3.1, como não temos impulso, pois $t \in$ $\left(t_{k}, t_{k+1}\right]$, obtemos

$$
\left\|U\left(t, t_{k}\right)\right\|_{i} \leq e^{\int_{t_{k}}^{t} \| A(s) \mid d s}
$$

Do mesmo modo provamos que

$$
\left\|U\left(t_{j+1}, t_{j}\right)\right\| \leq e^{\int_{t j}^{t_{j}-1} \mid A(s) \| d s}
$$


Portanto, de (4.3) e utilizando as hipóteses (i) e (ii), temos

$$
\|U(t, s)\| \leq e^{\int_{s}^{t} .|A(u)| d u}\left\|Q_{k}\right\| \ldots\left\|Q_{i+-1}\right\| \leq K e^{M(t-s)} e^{k-(i+1)+1} \leq K e^{M(t-s)} e^{k-i}
$$

Seja $s \leq t \leq s+h$. Como $h$ é a limitação inferior para a distância entre duas descontinuidades consecutivas, segue que o número de descontinuidades entre $s$ e $t$ é no máximo um. Em vista disto e de (4.4), temos

$$
\|U(t, s)\| \leq(K e) e^{M(t-s)}=K_{1} e^{M(t-s)}
$$

para $s \leq t \leq s+h$, onde $K_{1}=K e$.

Para $s \leq t$ qualquer, existe um inteiro positivo $k$ tal que $t \in[s+k h, s+(k+1) h]$. Assim

$$
\|U(t, s)\| \leq\|U(t, s+k h)\|\|U(s+k h, s+(k-1) h)\| \ldots\|U(s+h, s)\| \leq K_{1}^{k+1} e^{M(t-s)}
$$

Fazendo $\beta=h^{-1} \ln K_{1}$, temos $K_{1}^{k}=e^{\beta k h} \leq e^{\beta(t-s)}$. Portanto

$$
\|U(t, s)\| \leq K_{1} e^{(\beta+M)(t-s)} \leq K_{1} e^{\alpha(t \cdots, s)}, \quad s \leq t
$$

onde $K_{1}=K e$ e $\alpha=\beta+M=h^{-1} \ln K_{1}+M$. Analogamente estudamos o caso $s>t$.

Em [2], o autor observa, sem contudo provar, que o resultado abaixo é verdadeiro. Este resultado nos diz que, sob certas condições sobre $A(t)$ e sobre os operadores de impulso, $Q_{n}$, com $n \in \mathbb{Z}$ tal que $t_{n} \in J$, a EDI (3.1), (3.2) tem crescimento limitado.

Teorema 4.3 Consideremos a EDI (3.1), (3.2) e suponhamos que

(i) A seja integralmente limitada sobre J;

(ii) $\operatorname{det} Q_{n} \neq 0$, para $n \in \mathbb{Z}$ tal que $t_{n} \in J$.

(iii) Existam constantes $L \geq 0$ e $\lambda \in \mathbb{R}$ tais que os operadores de impulso $Q_{n}$ satisfaçam a condição,

$$
\prod_{\tau<t_{j} \leq t}\left\|Q_{j}\right\| \leq L e^{\lambda(t \cdot \tau)}, \quad t, \tau \in \mathbb{R}
$$


Então a EDI (3.1), (3.2) terá crescimento limitado.

Demonstração: Suponhamos que $A$ scja integralmente limitada sobre $J$. Então existirá uma constante $M>0$ tal que

$$
\sup _{t \in \mathbb{D}} \int_{t}^{t+1}: \mid A(\tau) \| d \tau \leq M
$$

Sejam $s, t \in J$ com $s \leq t$. Então existem inteiros $k$ e $i$, com $i \leq k$, tais que $s \in\left(t_{i}, t_{i+1}\right]$ e $t \in\left(t_{k}, t_{k-1}\right]$. Assim, temos

$$
\|U(t, s)\| \leq\left\|U\left(t, t_{k}\right)\right\|\left\|U\left(t_{k}, t_{k-1}\right)\right\| \ldots\left\|U\left(t_{i+1}, s\right)\right\|\left\|Q_{k}\right\|\left\|Q_{k-1}\right\| \ldots\left\|Q_{i+1}\right\| .
$$

Utilizando o Lema 3.1 , temos

$$
\begin{aligned}
\|U(t, s)\| & \leq e^{\int_{t_{k}}^{t} \| A(\tau) \mid d \tau} e^{\int_{t_{k-1}}^{t_{k}} \mid ' A(\tau) \| d \tau} \ldots e^{f_{s}^{t_{i}+1} \mid \cdot A(\tau) \| d \tau} \prod_{s \leq t_{j}<t}\left\|Q_{j}\right\|_{i}= \\
& =e^{f_{s}^{t}\|A(\tau)\| d \tau} \prod_{s \leq t_{j}<t}\left\|Q_{j}\right\| \leq e^{M(t-s)} L e^{\lambda(t-s)}= \\
& =L e^{(M+\lambda)(t-s)}=L e^{\mu(t-s)}, \quad s \leq t,
\end{aligned}
$$

onde $\mu=M+\lambda$.

Provamos o caso $s>t$ analogamente e, portanto, a EDI (3.1), (3.2) tem crescimento limitado sobre $J$.

Denotaremos por $\mathscr{T}\left(\left(A(t), Q_{n}\right),\left\{t_{n}\right\}_{n \subset \mathbb{Z},}, \delta, H\right)$ o conjunto de todas as EDIs (3.25), (3.26) cujos operadores de Cauchy, $V(t, \tau)$, satisfazem a seguinte condição:

para qualquer $s \in \mathbb{R}$, existe $\tau \in \mathbb{R}$ tal que a seguinte desigualdade é válida:

$$
\|V(t+s, u+s)-U(t+\tau, u+\tau)\|<\delta
$$

para $u, t \in[-H, H]$, onde $U(t, \tau)$ é o operador de Cauchy da EDI (3.1), (3.2).

Assim, $\mathscr{N}\left(\left(A(t), Q_{n}\right),\left\{t_{n}\right\}_{n \in \mathbb{Z}}, \delta, H\right)$ representa o conjunto das EDIs cujos operadores de Cauchy estão em uma "vizinhança" do operador de Cauchy da EDI (3.1), (3.2). 
No teorema a seguir, encontramos uma estimativa para a solução $x(t)$ da EDI (3.1), (3.2) usando desigualdades integrais para funções contínuas por partes. Este resultado será utilizado na demonstração do Teorema 4.5 .

Teorema 4.4 [[6], Teorema 2.3] Assumamos que existam constantes $K>1$ e $\alpha>0$ tais que

$$
\left\|e^{t \Lambda(u)}\right\| \leq K e^{\alpha t}
$$

para quaisquer $u, t \in \mathbb{R}$. Então, para qualquer solução $x(t)$ da EDI (3.1), (3.2), teremos a estimativa

$$
|x(t)| \leq\left(\prod_{t_{0}<t_{n}<t}\left\|Q_{n}\right\|\right) e^{\alpha\left(t-t_{0}\right)} K^{j(t)-j\left(t_{0}\right)+1} e^{\int_{t_{0}}^{t} K\|A(\tau)-A(u)\| d \tau},
$$

parat $\in\left(t_{k}, t_{k+1}\right]$, onde $j(t)=k$

Demonstração: Primeiramente, reescreveremos a EDI (3.1), (3.2) do seguinte modo

$$
\begin{gathered}
\frac{d x}{d t}=A(u) x(t)+[A(t)-A(u)] x(t), \quad t \neq t_{n}, \\
x\left(t_{n}^{+}\right)=Q_{n} x\left(t_{n}\right), \quad n \in \mathbb{Z},
\end{gathered}
$$

Podemos observar que a EDI (4.7), (4.8) é uma perturbação da EDI seguinte:

$$
\begin{gathered}
\frac{d y}{d t}=A(u) y(t), \quad t \neq t_{n}, \\
y\left(t_{n}^{+}\right)=Q_{n} y\left(t_{n}\right), \quad n \in \mathbb{Z} .
\end{gathered}
$$

Utilizando a Fórmula da Variação das Constantes para EDIs (Lema 2.1), a solução $x(t)$ da EDI (4.7), (4.8) é dada por

$$
x(t)=U(t, s) x(s)+\int_{s}^{t} U(t, \tau)[A(\tau)-A(u)] x(\tau) d \tau
$$


onde $U(t, s)=U(t) U^{-1}(s)$, com $U(t)$ sendo a matriz de fundamental da EDI (4.9), (4.10) dada por

$$
U(t)=e^{\left(t-t_{k}\right) A(u)} \prod_{t_{0}<t_{n}<t} Q_{n} e^{\left(t_{n}-t_{n-1}\right) A(u)},
$$

para $t \in\left(t_{k}, t_{k+1}\right]$

De (4.11) e (4.12), segue que

$$
\begin{aligned}
x(t) & =e^{\left(t-t_{k}\right) A(u)}\left(\prod_{s<t_{n}<t} Q_{n} e^{\left(t_{n}-t_{n-1}\right) A(u)}\right) e^{\left(t_{i} \cdots-s\right) A(u)} x(s)+ \\
& +\int_{s}^{t} e^{\left(t-t_{k}\right) A(u)}\left(\prod_{\tau<t_{n}<t} Q_{n} e^{\left(t_{n}-t_{n-1}\right) A(u)}\right) e^{\left(t_{j}(\tau) \cdots \tau\right) A(u)}[A(\tau)-A(u)] x(\tau) d \tau,
\end{aligned}
$$

onde $t \in\left(t_{k}, t_{k+1}\right], s \in\left(t_{i}, t_{i+1}\right]$, com $s \leq t$ e $i \leq k$, e $j(\tau)$ é tal que $\tau \in\left(t_{j(\tau)}, t_{j(\tau)+1}\right]$. Utilizando a desigualdade (4.6) em (4.13), segue que

$$
\begin{aligned}
&|x(t)| \leq K^{j(t)-j(s)+1} e^{\alpha(t-s))} \prod_{s<t_{n}<t}\left\|Q_{n}\right\||x(s)|+ \\
&+\int_{s}^{t} K^{j(t)-j(s)+1} \prod_{\tau<t_{n}<t}\left\|Q_{n}\right\| e^{\alpha(t-\tau)}\|A(\tau)-A(u)\| x(\tau) \mid d \tau .
\end{aligned}
$$

Seja $H$ fixado tal que $t_{0} \leq s<t \leq H$. Então, de (4.14), temos

$$
\begin{aligned}
\prod_{t_{0}<t_{n}<H}\left\|Q_{n}\right\| e^{-\alpha t}|x(t)| K^{j(t)} & \leq K K^{-j(s)} \prod_{s<t_{n}<H} \| Q_{n}|| e^{-\alpha s}|x(s)|+ \\
& +\int_{s}^{t} K \prod_{\tau<l_{n}<H}\left\|Q_{n}\right\| e^{-\alpha \tau} K^{-j(\tau)}\|A(\tau)-A(u)\| x(\tau) \mid d \tau .
\end{aligned}
$$

Agora, definamos

$$
\varphi(t)=\prod_{i<t_{n}<H}\left\|Q_{n}\right\| e^{-\alpha t} K^{-j(t)}|x(t)| .
$$

De (4.15) e (4.16) obtemos

$$
\varphi(t) \leq K \varphi(s)+\int_{s}^{t} K \varphi(\tau)\|A(\tau)-A(u)\| x(\tau) \mid d \tau
$$


e utilizando o Lema 3.1 no intervalo $t_{i}<s<t<t_{i+1}$, segue que

$$
\varphi(t) \leq K \varphi(s) e^{\int_{s}^{t} K|A(\tau)-A(u)| ! d \tau}
$$

Portanto,

$$
\varphi(t) \leq K \varphi\left(t_{i}^{+}\right) e^{\int_{t_{i}}^{t} K|\cdot A(\tau)-A(u)| \mid d \tau}
$$

para $t \in\left(t_{i}, t_{i+1}\right)$. De (4.16), temos

$$
\frac{\varphi\left(t_{i}^{+}\right)}{\varphi\left(t_{i}\right)}=\frac{1}{K\left\|Q_{i}\right\|} \frac{\left|x\left(t_{i}^{+}\right)\right|}{\left|x\left(t_{i}\right)\right|}
$$

e de (3.2), temos $\left|x\left(t_{i}^{+}\right)\right| \leq \| Q_{i}|| x\left(t_{i}\right) !$. Deste modo, (4.18) implica que

$$
\varphi\left(t_{i}^{\top}\right) \leq K^{-1} \varphi\left(t_{i}\right)
$$

Daí, substituindo (4.19) em (4.17), segue que

$$
\varphi(t) \leq \varphi\left(t_{i}\right) e^{K \int_{t_{i}}^{t}\|A(\tau)-A(u)\| d \tau}
$$

Pode ser facilmente verificado que

$$
\varphi\left(t_{i}\right) \leq K \varphi\left(t_{0}\right) e^{K \int_{i_{0}}^{t}|| A(\tau)-A(u) \mid d \tau}
$$

Portanto

$$
\varphi(t) \leq K \varphi\left(t_{0}\right) e^{K \int_{t_{0}}^{t}\|A(\tau)-A(u)\| d \tau},
$$

isto é,

$$
\prod_{t<t_{n}<H}\left\|Q_{n}\right\| e^{-\alpha t} K^{-j(t)}\left|x(t) ! \leq \prod_{t_{0}<t_{n}<H}\left\|Q_{n}\right\| e^{-\alpha t_{0}} K^{-j\left(t_{0}\right)}\right| x\left(t_{0}\right) \mid e^{K \int_{t_{0}}^{t} \mid \cdot A(\tau)-A(u) \| d \tau},
$$

ou ainda,

$$
|x(t)| \leq \prod_{t_{0}<t_{n}<t}|| Q_{n} \| e^{\alpha\left(t-t_{0}\right)} K^{j(t)-j\left(t_{0}\right)+1} e^{\int_{t_{0}}^{t} K\|A(\tau)-A(u)\| d \tau}\left|x\left(t_{0}\right)\right|
$$

e a demonstração está terminada. 
Observação 4.1 Segue da demonstraçāo do Teorema 4.4 que, dados $t \in\left(t_{k}, t_{k+1}\right]$ e $s \in\left(t_{i}, t_{i+1}\right]$, com $s \leq t$, a solução $x(t)$ da EDI (3.1), (3.2) satisfaz a seguinte desigualdade

$$
|x(t)| \leq \prod_{s<t_{n}<t}\left|Q_{n} \| e^{\alpha(t-s)} K^{j(t)-j(s)+1} e^{\int_{s}^{t} K\|: A(\tau)-A(u)\| d \tau}\right| x(s) \mid .
$$

No teorema a seguir, considerando a EDI (3.1), (3.2) sobre $\mathbb{R}$, apresentamos condições suficientes sobre $A(t)$, sobre os operadores de impulso, $Q_{n}, n \in \mathbb{Z}$, e sobre a seqüência de tempos de impulso $\left\{t_{n}\right\}_{n \in \mathbb{Z}}$ a fim de obtermos (4.2), ou seja, a fim de que a EDI (3.1), (3.2) tenha crescimento limitado.

Teorema 4.5 [[6], Teorema 2.4] Consideremos a EDI (3.1), (3.2) sobre $\mathbb{R}$ e suponhamos que

(i) Existam constantes $K>1$ e $\alpha>0$ tais que $\left\|e^{t A(u)}\right\| \leq K e^{\alpha t}$, para quaisquer $t, u \in \mathbb{R}$;

(ii Exista $L>0$ tal que ||$A\left(t_{1}\right)-A\left(t_{2}\right) \| \leq L\left|t_{1}-t_{2}\right|$, para quaisquer $t_{1}, t_{2} \in \mathbb{R}$;

(iii) $\operatorname{det} Q_{n} \neq 0$, para $n \in \mathbb{Z}$.

(iv) Existam constantes $K^{\prime} \geq 1$ e $\beta>0$ tais que

$$
\prod_{s<l_{n}<t}\left\|Q_{n}\right\| \leq K^{\prime} e^{\beta(j(t)-j(s))} \quad e \quad \prod_{s<t_{n}<t}\left\|Q_{n}^{-1}\right\| \leq K^{\prime} e^{\beta(j(t)-j(s))},
$$

para quaisquer $s, t \in \mathbb{R}$;

(iv) Exista $h>0$ tal que $h<t_{n+1}-t_{n}$, para todo $n \in \mathbb{Z}$.

Então

$$
\|U(t, s)\| \leq M e^{\gamma^{\prime}|t-s|},
$$

para quaisquer $s, t \in \mathbb{R}$, com $s \leq t$.

Demonstração: Sejam $s, t \in \mathbb{R}, \operatorname{com} s \leq t$. Então existirão inteiros $k$ e $i$, com $i \leq k$, tais que $s \in\left(t_{i}, t_{i+1}\right]$ e $t \in\left(t_{k}, t_{k+1}\right]$. 
Seja $x(t)$ uma solução da EDI (3.1), (3.2). Então, pela Observação 4.1 c utilizando (ii) em (4.20), obtemos

$$
|x(t)| \leq K^{j(t)-j(s)-1} \prod_{s<t_{n}<t}\left\|Q_{n}\right\| e^{\alpha(t-s)} e^{K L \int_{s}^{t}|\tau-u| d \tau}|x(s)|
$$

Fazendo $u=(s+t) / 2$, podemos reescrever (4.21) como

$$
|x(t)| \leq K^{j(t)-j(s)+1} \prod_{s<l_{n}<l}\left\|Q_{n}\right\| e^{\alpha(t-s)} e^{K L(t-s)^{2} / 4}|x(s)| .
$$

e utilizando (iv) segue que

$$
\left|x(t) i \leq K^{j(t)-j(s)+1} K^{\prime} e^{\beta(j(t)-j(s) j} e^{\alpha(t-s)} e^{K L(t-s)^{2} / 4}\right| x(s) \mid .
$$

Daí, substituindo $K^{2} K^{\prime} e^{\beta}$ por $M$ e utilizando $(v)$, segue que

$$
|x(t)| \leq M e^{(\alpha+M L(t-s) / 4)(t-s)}|x(s)|
$$

para $s \leq t \leq s+\delta$

Sejam $h_{1}=2\{(\ln M) / L M\}^{1 / 2}, \gamma=(1 / 2)(L M \ln M)^{1 / 2}$ e $h=\min \left\{\delta, h_{1}\right\}$. Então, para $s \leq t \leq$ $s+h$, temos

$$
|x(t)| \leq M e^{(\alpha+\gamma)(t-s)}|x(s)| .
$$

De modo geral, temos $s+n h \leq t \leq s+(n+1) h$, para algum $n \in \mathbb{Z}$. Então,

$$
\begin{aligned}
|x(t)| & \leq M e^{(\alpha+\gamma)(t-s-n h)}|x(s+n h)| \leq \\
& \leq M^{2} e^{(\alpha-\gamma)(t-s-(n-1) h)}|x(s-(n-1) h)| \leq \ldots \leq M^{n-1} e^{(\alpha+\gamma)(t-s)}|x(s)| .
\end{aligned}
$$

Observemos que $M^{n}=e^{n \ln M} \leq e^{\ln M(t-s) / h}$, deste modo,

$$
|x(t)| \leq M e^{(\alpha+\gamma+\ln M / h)\langle t-s\rangle}|x(s)|,
$$

para $s \leq t$ e, portanto,

$$
\|U(t, s):\| \leq M e^{\gamma(t-s)},
$$


para $s \leq t$, onde $\gamma^{\prime}=\alpha+\frac{1}{2}(L M \ln M)^{1 / 2}+\ln M / h$

Analogamente, estudamos o caso $s>t$. Para este caso, utilizamos a hipótese

$$
\prod_{t<t_{n}<s}\left\|Q_{n}^{-1}\right\| \leq K^{\prime} e^{\beta(j(t)-j(s))}
$$

Isto encerra a demonstração.

\subsection{Relações entre Crescimento Limitado e Dicotomia Exponen- cial}

Nesta seção, discutimos uma relação entre dicotomia e crescimento limitado para a EDI (3.1), (3.2).

Em [6], os autores enunciam sem contudo demonstrar o próximo teorema. Neste teorema a condição (4.22) a seguir é somente uma condição necessária para a existência de dicotomia exponencial para a EDI (3.1), (3.2).

Teorema 4.6 [[6], Teorema 4.2] Consideremos a EDI (3.1), (3.2) e suponhamos que $\operatorname{det} Q_{n} \neq 0$, para $n$ tal que $t_{n} \in J$. Suponhamos, ainda, que a EDI (3.1), (3.2) tenha uma dicotomia exponencial sobre $\mathbb{R}$. Então, para qualquer $\theta \in(0,1)$, existirá $H>t_{0}$ tal que

$$
|x(s)| \leq \theta \sup \{|x(u)|:|u-s| \leq H\}, \quad s \leq H, s \in J .
$$

Demonstração: Como a EDI (3.1), (3.2) tem uma dicotomia exponencial sobre $J$, segue que existem uma projeção $P: X \rightarrow X$ e constantes positivas $K$ e $\alpha$ tais que

$$
\| U(t) P U^{--1}(s) i \mid \leq K e^{-\alpha(t-s)} \quad s \leq t
$$

$\mathrm{e}$

$$
\left\|U(t)(I-P) U^{-1}(s)\right\| \leq K e^{-\alpha(s-i)} \quad s \geq t
$$


Seja $x(t)$ uma solução qualquer da EDI (3.1), (3.2). Definamos

$$
x_{1}(t)=U(t) P U^{-1}(t) x(t)
$$

e

$$
x_{2}(t)=U(t)(I-P) U^{-1}(t) x(t)
$$

Assim, 


$$
x(t)=U(t) P U^{-1}(s) x_{1}(s)+U(t)(I-P) U^{-1}(s) x_{2}(s) .
$$

Suponhamos que $\left\{x_{1}(s)|\leq| x_{2}(s) \mid\right.$. Então, para $s \leq t$, temos

$$
\begin{aligned}
|x(t)| & \geq\left\|U(t)(I-P) U^{-1}(s)||\left|x_{2}(s)\right|-|| U(t) P U^{-1}(s)\right\|\left|x_{1}(s)\right| \geq \\
& \geq K^{-1} e^{\alpha(t-s)} x_{2}(s)\left|-K e^{-\alpha(t-s)}\right| x_{1}(s)\left|\geq\left[K^{-1} e^{\alpha(t-s)}-K e^{-\alpha(t-s)}\right]\right| x_{2}(s) \mid \geq \\
& \geq \frac{1}{2}\left[K^{-1} e^{\alpha(t-s)}-K e^{-\alpha(t-s)}\right]|x(s)| .
\end{aligned}
$$

Pelo mesmo raciocínio, se supusermos que $\left|x_{1}(s)\right| \geq\left|x_{2}(s)\right|$ então, para $s \geq t$, teremos

$$
|x(t)| \geq \frac{1}{2}\left[K^{-1} e^{\alpha(t-s)}-K e^{-\alpha(t-s)}\right]|x(s)| .
$$

Observemos que, para qualquer $\theta \in(0,1)$, podemos escolher $H>0$ tão grande de modo que tenhamos

$$
K^{-1} e^{\alpha H}-K e^{-\alpha H} \geq 2 \theta^{-1}
$$

e, portanto

$$
|x(s)| \leq \theta \sup \{|x(u)|:|u-s| \leq H\}, \quad s \leq H .
$$

Isto completa a demonstração.

O próximo teorema apresenta um critério de suficiência para que a EDI (3.1), (3.2) tenha uma dicotomia exponencial sobre $\left[t_{0}, \infty\right)$.

Teorema 4.7 [/6], Teorema 4.3] Consideremos a EDI (3.1), (3.2) sobre a semi-reta $J=\left[t_{0}, \infty\right) e$ suponhamos que $\operatorname{det} Q_{n} \neq 0$, para $n \in \mathbb{N}$. Suponhamos que existam constantes $H>t_{0}, C>1 e$ $\theta \in(0,1)$ tais que toda solução $x(t)$ da EDI (3.1), (3.2) satisfaça

$$
|x(t)| \leq C|x(s)|, \quad \text { para } t_{0} \leq s \leq t \leq s+H
$$

e

$$
|x(s)| \leq \theta \sup \{|x(u)|:|u-s| \leq H\}, \quad \text { para } s \leq H .
$$


Suponhamos, também, que toda solução não-limitada x(t) da EDI (3.1), (3.2) satisfaça a seguinte hipótese:

Para qualquer $t \geq t_{0}$ e para qualquer inteiro positivo $m$, se tivermos $\left|x\left(t^{++}\right)\right|>C \theta^{-m}$ então existirá um número $\tau(t)>t$ tal que

$$
\begin{gathered}
0<\tau(t)-t<H \\
C \theta^{-(m+1)}<\left|x\left(\tau(t)^{+}\right)\right|<C \theta^{-(m+2)} \quad \text { e } \quad C \theta^{-m}<|x(\tau(t))| \leq C / \theta^{-(m+1)} .
\end{gathered}
$$

Suponhamos, ainda, que exista uma constante positiva $M$ tal que $\left\|Q_{n}\right\| \leq M$, para todo $n \in \mathbb{N}$. Então a EDI (3.1), (3.2) terá uma dicotomia exponencial sobre $\left[t_{0}, \infty\right)$.

Demonstração: Primeiramente, suponhamos que $x(t)$ seja uma solução limitada da EDI (3.1), (3.2). Fntão

$$
m(s)=\sup \{|x(u)|: u \geq s\}<\infty
$$

Seja $t \geq s+H$, com $H \geq 0$. De (4.24), observamos que $|x(t)| \leq \theta m(s)$. Assim,

$$
m(s)=\sup \{|x(u)|: s \leq u \leq s+H\}
$$

Scja $t_{0} \leq s \leq t<\infty$. Então, utilizando (4.23), (4.25) e o fato de que $\theta \in(0,1)$, temos

$$
|x(t)| \leq \theta m(s)<m(s)=\sup \{|x(u)|: s \leq u \leq s+H\} \leq C|x(s)| .
$$

Seja $n$ um inteiro não-negativo tal que $s+n H \leq t<s+(n+1) H$. Então

$$
\begin{aligned}
|x(t)| & \leq \theta \sup \left\{\left|x\left(u_{1}\right)\right|: t-H \leq u_{1} \leq t+H\right\} \leq \\
& \leq \theta \sup \left\{\left|x\left(u_{1}\right)\right|: s+(n-1) H \leq u_{1} \leq t+H\right\} \leq \\
& \leq \theta^{2} \sup \left\{\left|x\left(u_{2}\right)\right|: s+(n-2) H \leq u_{2} \leq t+2 H\right\} \leq \\
& \leq \cdots \leq \theta^{n} C|x(s)|=\frac{C}{\theta} \theta^{n+1}|x(s)| \leq \frac{C}{\theta} \theta^{(t-s) / H}|x(s)|
\end{aligned}
$$


pois $n+1>\frac{t-s}{H}$. Fazendo $K=C / \theta$ e $\alpha=-\ln \theta / H>0$, concluímos que

$$
x(t)\left|\leq K e^{[(t-s) / H ! \ln \theta}\right| x(s)\left|=K e^{-\alpha(s-t)}\right| x(s) \mid,
$$

para $t_{0} \leq s \leq t$

Agora, suponhamos que $x(t)$ seja uma solução não-limitada da EDI (3.1), (3.2), com $\left|x\left(t_{0}\right)\right|=1$. Então existirá um $\tau_{1}>0$ tal que $\left|x\left(\tau_{1}\right)^{!}>1 \mathrm{e}: x\left(\tau_{1}^{+}\right)\right|>C / \theta$. Assim, a hipótese sobre soluções não-limitadas implicará na existência de um número $\tau\left(\tau_{1}\right)=\tau_{2}$ tal que

$$
0<\tau_{2}-\tau_{1}<H, \quad C \theta^{-2}<\left|x\left(\tau_{2}^{+}\right)\right|<C \theta^{-3} \quad \text { e } \quad C \theta^{11}<\left|x\left(\tau_{2}\right)\right| \leq C \theta^{-2} .
$$

Utilizando o mesmo raciocínio, segue que existirá um número $\tau\left(\tau_{2}\right)=\tau_{3}>0$ tal que

$$
0<\tau_{3}-\tau_{2}<H, \quad C \theta^{-3}<\left|x\left(\tau_{3}^{+}\right)\right|<C \theta^{-4} \quad \text { e } \quad C \theta^{2}<\left|x\left(\tau_{3}\right)\right| \leq C \theta^{-3} .
$$

Continuando com o argumento acima, encontramos uma sequiência $\left\{\tau_{i}\right\}$, com $0<\tau_{i+1}-\tau_{i}<H$ e tal que

$$
C \theta^{-i}<\left|x\left(\tau_{i}^{+}\right)\right|<C \theta^{(i \mid 1)} \quad \text { e } \quad C \theta^{-(i-1)}<\left|x\left(\tau_{i}\right)\right| \leq C \theta^{-i} .
$$

Suponhamos que $i(m)$ seja o índice tal que $\tau_{m} \in\left(t_{i(m)+1}, t_{i(m)+2}\right]$. Sejam $s, t \in \mathbb{R}$ tais que $\tau_{m}<t \leq \tau_{m+1}$ e $\tau_{n}<s \leq \tau_{n+1}$, com $s \geq t$. Assim, temos

$$
\left|x\left(\tau_{m}\right)\right| \leq C \theta^{-m}
$$

Para $\tau_{m}<t \leq \tau_{m+1} \leq \tau_{m}+H$, a condição de crescimento limitado implica que

$$
|x(t)| \leq C\left|x\left(\tau_{m}^{+}\right): \leq C\right|\left|Q_{i(m)+1}\left\|x\left(\tau_{m}\right)\left|\leq C^{2} \theta^{-m}\right| \mid Q_{i(m)-1}\right\|\right.
$$


e, para $\tau_{n}<s \leq \tau_{n+1} \leq \tau_{n}+H$, a condição de crescimento limitado implica que

$$
\left|x\left(\tau_{n-1}\right)\right| \leq C|x(s)|
$$

De (4.26), segue que

$$
C \theta^{-(n+1)}<\left|x\left(\tau_{n+1}^{\cdot}\right)\right| \leq \| Q_{i(n)+1}||\left|x\left(\tau_{n+1}\right)\right|
$$

e, portanto

$$
C<\theta^{n+1}\left\|Q_{i(n)+1}\right\| ! x\left(\tau_{n+1}\right) \mid
$$

Assim, de (4.27) e (4.29), temos

$$
|x(t)| \leq C \theta^{-m} \theta^{n+1}|| Q_{i(m)+1}\|\| Q_{i(n)+1} \||| x\left(\tau_{n+1}\right) !
$$

Daí, utilizando (4.28) em (4.30), obtemos

$$
|x(t)| \leq C^{2} \theta^{n-m+1}\left\|Q_{i(m)+1}\left|\| Q_{i(n)+1}\right||x(s)| \leq C^{2} M^{2} \theta^{n-m+1}|x(s)| \leq C^{2} M^{2} \theta^{(s-t) / H}|x(s)|,\right.
$$

pois $(t-s)<(n-m+1) H$. Portanto

$$
\left|x(t) ! \leq K e^{-\alpha(s \cdot t)}\right| x(s) \mid
$$

para $\tau_{1} \leq t \leq s, \operatorname{com} K=C^{2} M^{2}$ e $\alpha=-\ln \theta / H>0$.

Seja $X_{1}$ o subespaço de todos os $x(\xi) \in X$ para os quais a função $U(t) x(\xi), \operatorname{com} t \geq \xi$, ć limitada e scja $X_{2}$ um subespaço de $X$ complementar a $X_{1}$.

Seja $\xi \in X_{2}, \operatorname{com}|\xi|=1$. Então a solução $x(t)=x\left(t ; t_{0}, \xi\right)$ da EDI (3.1), (3.2) iniciando em $\left(t_{0} . \xi\right)$ é não-limitada com $\left|x\left(t_{0}\right)\right|=|\xi|=1$. Assim, existirá $u>t_{0}$ tal que $\mid x\left(u ; t_{0}, \xi\right)_{i} \geq C \theta^{-1}$. Seja $\tau_{1}(\xi)$ o mínimo de todos estes pontos $u$. Então $\left|x\left(\tau_{1}^{+}\right)\right| \geq C \theta^{-1}$.

Agora, mostraremos que o conjunto

$$
D=\left\{\tau_{1}(\xi): \xi \in X_{2},|\xi|=1\right\}
$$


é limitado. Suponhamos, por absurdo, que $D$ não seja limitado. Então existirá uma sequiência $\left\{\xi_{v}\right\}$ em $X_{2}$ tal que $\left|\xi_{v}\right|=1$ e $\tau_{1}\left(\xi_{v}\right) \rightarrow+\infty$ quando $v \rightarrow+\infty$. Como $\left\{\xi_{v}\right\}$ é uma seqüência sobre a esfera unitária de $X$, podemos assumir, sem perda de generalidade, que $\xi_{v} \rightarrow \xi$ quando $v \rightarrow+\infty$, com $\left|\xi_{v}\right|=1 \mathrm{e} \xi_{v} \in X_{2}$. Também temos que $x\left(t ; t_{0}, \xi_{v}\right) \rightarrow x\left(t ; t_{0}, \xi\right)$ quando $v \rightarrow+\infty$.

Se $t_{0} \leq t<\tau_{1}\left(\xi_{v}\right)$, então $\left|x\left(t ; t_{0}, \xi\right)\right| \leq C \theta^{-1}$. Seja $t$ um número positivo arbitrariamente grande. Como $\xi_{v} \rightarrow \xi$ quando $v \rightarrow+\infty$, segue que existe um inteiro positivo $k=k(t)$ tal que $v \geq k$ implica que $t_{0} \leq t<\tau_{1}\left(\xi_{v}\right)$. Então $\left|x\left(t ; t_{0}, \xi_{v}\right)\right|<C \theta^{-1}$. Disto e pela convergência $x\left(t ; t_{0}, \xi_{v}\right) \rightarrow x\left(t ; t_{0}, \xi_{)}\right)$, obtemos $\left|x\left(t ; t_{0}, \xi\right)\right|<C \theta^{-1}$, para todo $t \geq t_{0}$, isto é, $x\left(t ; t_{0}, \xi\right)$ é limitada. Isto contradiz os fatos de que $\xi \in X_{2}$ e que toda solução com valor inicial em $X_{2}$ é não-limitada. Então o conjunto $D$ é necessariamente limitado

Seja $S=\sup D$. Então $t_{0}<S<+\infty$. De (4.31), para $S \leq t \leq s<+\infty$, temos

$$
\left|x\left(t ; t_{0}, \xi\right)\right| \leq K e^{-\alpha(s \cdot t)}\left|x\left(s ; t_{0}, \xi\right)\right| .
$$

Observemos a seguinte diferença na obtenção de (4.31) e de (4.32): em (4.31), $\tau_{1}$ depende do valor inicial de $x(t)$, enquanto que $\mathrm{cm}$ (4.32), $S$ é independente do valor inicial de $x(t)$.

Seja $P$ uma projeção ortogonal sobre $X_{1}$. Então $(I-P) X=X_{2}$ e $X=X_{1} \oplus X_{2}$. Para cada $t$ fixado, consideremos

$$
x_{1}(t)=U(t) P U^{-1}(t) x(t) \in X_{1}
$$

$\mathrm{e}$

$$
x_{2}(t)=U(t)(I-P) U^{-1}(t) x(t) \in X_{2} .
$$

Assim,

$$
x(t)=x_{1}(t)+x_{2}(t)-U(t) P U^{-1}(s) x_{1}(s)+U(t)(I-P) U^{-1}(s) x_{2}(s) .
$$

Por (4.32), segue que

$$
\|U(t, s)\| \leq K e^{-\alpha(t-s)} ; \quad s \leq t .
$$


Portanto,

$$
\begin{aligned}
\left|U(t) P U^{-1}(s) x(s)\right| & \because-\left|U(t, s) x_{1}(s) ! \leq K e^{-\alpha(t-s)}\right| x_{1}(s)\left|\leq K e^{-\alpha(t-s)}\right| P x(s) \mid \leq \\
& \leq K e^{-\alpha(t-s)|!| P \mid}|x(s)|
\end{aligned}
$$

donde segue que

$$
\left\|U(t) P U^{-1}(s)\right\| \leq K e^{-\alpha(t \cdots s)}|| P \mid !, \quad s \leq t .
$$

Analogamente,

$$
\left\|U(t)(I-P) U^{-1}(s)\right\| \leq K e^{-\alpha(s-1)} \mid I I-P \|, \quad s \geq t .
$$

Logo

$$
\left\|U(t) P U^{-1}(s)\right\| \leq \tilde{K} e^{-\alpha(t-s)}, \quad s \leq t
$$

$\mathrm{e}$

$$
\left|U(t)(I-P) U^{-1}(s)\right| \leq \tilde{K} e^{-\alpha(t-s)}, \quad s \geq t
$$

onde $\tilde{K}=\max \{K\|P\|, K|| I-P \|\}$. Deste modo, o teorema está provado.

O teorema a seguir nos diz que se a EDI (3.1), (3.2) tiver crescimento limitado, entāo toda EDI em uma "vizinhança" de seu operador de Cauchy terá crescimento limitado.

Teorema 4.8 //2], Teorema 1] Consideremos a EDI (3.1), (3.2) sobre $\mathbb{R}$ e suponhamos que $\operatorname{det} Q_{n} \neq$ 0 , para $n \in \mathbb{Z}$. Suponhamos que a EDI (3.1), (3.2) tenha crescimento limitado. Entāo cada EDI (3.25), (3.26) em. $\mathscr{A}\left(\left(A(t), Q_{n}\right),\left\{t_{n}\right\}_{n \in \mathbb{Z}}, \delta, H\right)$ também terá crescimento limitado.

Demonstração: Suponhamos que a EDI (3.25), (3.26) pertença a $\mathscr{N}\left(\left(A(t), Q_{n}\right),\left\{t_{n}\right\}_{n \in \mathbb{Z}}, \delta, H\right)$ (veja definição ao final do Teorema 4.3). Então, para qualquer $s \in \mathbb{R}$, existirá $\tau \in \mathbb{R}$ tal que, para $H \leq t, u \leq H$, tenhamos

$$
\| V(t+s, u+s)-\left.U(t+\tau, u-\tau)\right|_{i}<\delta
$$

onde $V(t . s)$ é o operador de Cauchy dá EDI (3.25), (3.26). 
Pelo fato da EDI (3.1), (3.2) ter crescimento limitado existem constantes $C \geq 1$ e $\lambda>0$ tais que

$$
i U(t+\tau, u+\tau) i \mid \leq C e^{\lambda|t-u|}
$$

e, utilizando (4.33), segue que

$$
\|V(t-s, u+s)\| \leq\|V(t, u)-U(t+\tau, u+\tau)\|+\| U(t+\tau, u+\tau) \mid \leq C e^{\lambda|t-u|}+\delta
$$

para $-H \leq t, u \leq H$. Como o número $s$ é arbitrário, tomando-o igual a zero, segue que

$$
|i V(t, u)| \mid \leq C e^{\lambda|t-u|}+\delta \leq(C+\delta) e^{\lambda . t-u},
$$

para $-H \leq t, u \leq H$.

Suponhamos que $t \geq u$. Então existe um número inteiro positivo $k$ tal que

$$
u+2 k H \leq t \leq u+2(k+1) H .
$$

Daí, por (4.34), temos

$$
\begin{aligned}
\| V(t, u)_{i} \mid & \leq|V(t, u+2 k H)|_{i}^{\mid}\|V(u+2 k H, u+2(k-1) H)\| \ldots\|V(u+2 H, u)\| \leq \\
& \leq(C+\delta)^{k+1} e^{\lambda(t-u)}=(C+\delta)(C+\delta)^{(1 / 2) H^{-1} 2 H k} e^{\lambda(t-u)} \leq \\
& \leq(C+\delta)(C+\delta)^{(1 / 2) H^{-1}(t-u)} e^{\lambda(t-u)}=(C+\delta) e^{\left[\lambda+(1 / 2) H^{-1} \ln (C+\delta)^{\prime}(t-u)\right.} .
\end{aligned}
$$

O caso $t<u$ é considerado analogamente. 


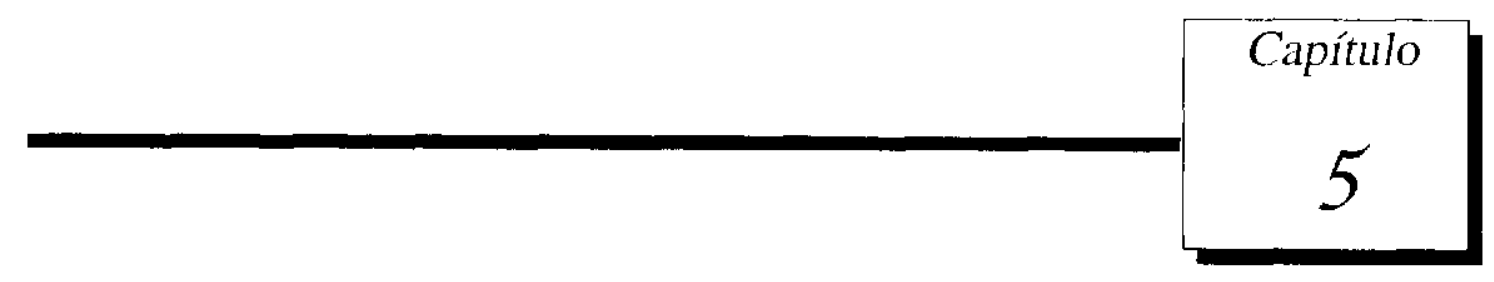

\section{Dicotomias e Admissibilidade}

Neste capítulo, estabelecemos relações entre admissibilidade para pares de espaços de funções e a existência de dicotomias exponencial ou ordinária para a EDI (3.1), (3.2) sobre $\mathbb{R}_{+}$. Consideramos certos espaços de funções limitadas. Em seguida, relacionamos dicotomia ordinária e admissibilidade. Finalmente, apresentamos dois resultados (Teoremas 5.3 e 5.4) que juntos dizem que se nossa EDI tiver uma dicotomia exponencial, então, dada certa perturbação limitada, obtemos uma EDI não-homogênea que admitirá solução também limitada, e vale a recíproca. O último teorema do capítulo refina este resultado e esta é nossa contribuição neste capítulo.

Consideremos a EDI não-homogênea

$$
\begin{gathered}
\frac{d x}{d t}=A(t) x+f(t), \quad t \neq t_{n}, \\
x\left(t_{n}^{1}\right)=x\left(t_{n}\right)+I_{n} x\left(t_{n}\right)+h_{n}=Q_{n} x\left(t_{n}\right)+h_{n}, \quad n \in \mathbb{N},
\end{gathered}
$$

onde

- $A: \mathbb{R}_{+} \rightarrow L(X)$ é uma função contínua por partes, com descontinuidade de primeira espécie em $t=t_{n}$, contínua à esquerda em $t=t_{n}, n \in \mathbb{N}$;

- $f: \mathbb{R}_{+} \rightarrow X$ é uma função contínua por partes, com descontinuidade de primeira espécie em $t=t_{n}$, contínua à esquerda $\mathrm{em} t=t_{n}, n \in \mathbb{N}$; 
- $I_{n}: X \rightarrow X$ é um operador contínuo, para qualquer $n \in \mathbb{N}$;

- $h_{n} \in X$, para qualquer $n \in \mathbb{N}$.

Consideremos, também, os seguintes espaços lineares:

$$
\mathscr{L}_{1}\left(\mathbb{R}_{i}, X\right)=\left\{f: \mathbb{R}_{+} \rightarrow X: \int_{0}^{\infty}|f(t)| d t<\infty\right\}
$$

$$
\ell_{1}(\mathbb{N})=\left\{h=\left\{h_{n}\right\}_{n \in \mathbb{N}}: \sum_{n=1}^{\infty}\left|h_{n}\right|<\infty\right\} .
$$

Podemos observar que os espaços $\mathscr{L}_{1}\left(\mathbb{R}_{+}, X\right)$ e $\ell_{1}(\mathbb{N})$ são espaços de Banach com respeito às normas

$$
\|f\|_{1}=\int_{0}^{\infty}|f(t)| d t
$$

e

$$
\left|i h \|_{1}=\sum_{j=1}^{\infty}\right| h_{n} \mid,
$$

respectivamente. Note que $\mathscr{L}_{1}\left(\mathbb{R}_{+}, X\right)$ é o espaço das funções de $\mathbb{R}_{+}$em $X$ absolutamente integráveis no sentido da integral imprópria de Riemann.

Seja $\mathscr{B}\left(\mathbb{R}_{+}, X\right)$ o espaço de todas as funções limitadas em $\mathbb{R}_{\text {. }}$ com valores em $X$ que são contínuas em $t \neq t_{n}$, têm descontinuidade de primeira espécie em $t=t_{n}$ e são contínuas à esquerda em $t=t_{n}$. Definamos, também, o espaço seguinte

$$
\mathscr{B}(\infty):-\left\{f \in \mathscr{B}\left(\mathbb{R}_{+}, X\right): \lim _{t \rightarrow+\infty} f(t) \text { cxiste }\right\}
$$

isto é, $\mathscr{B}(\infty)$ é o espaço de funções de $\mathscr{R}\left(\mathbb{R}_{+}, X\right)$ com limite no infinito. Os espaços $\mathscr{P}\left(\mathbb{R}_{+}, X\right)$ e $\not(\infty)$ munidos da norma

$$
\|x\|_{\infty}=\sup \left\{|x(t)|: t \in \mathbb{R}_{i}\right\}
$$

sĩo espaços de Banach. Além disso, se $F: \mathbb{R}_{+} \rightarrow L(X)$ e $F(t)$ for invertível para cada $t \in \mathbb{R}_{+}$, isto ¿́, cxiste $F^{-1}(t): X \rightarrow X$, para cada $t \subset \mathbb{R}_{+}$, então definimos

$$
\mathscr{B}_{F}(\infty):=\left\{f \in \mathscr{B}\left(\mathbb{R}_{+}, X\right): F^{-1} f \in \mathscr{B}(\infty)\right\}
$$


Em $\mathscr{B}_{F}(\infty)$, consideramos a norma

$$
\|f\|_{F}=\mid\left[F f \|_{\infty}\right.
$$

Se $F$ for limitada, então $\mathscr{h}_{F}(\infty)$ será um espaço de Banach.

Definição 5.1 Sejam $\mathscr{D}$ e $\mathscr{G}$ espaços de funções de $\mathbb{R}$ em X. Diremos que o par $(\mathscr{D}, \mathscr{G})$ é admissível para a EDI (5.1), (5.2) se, para cada f no espaço G, existir uma solução de (5.1), (5.2) pertencente $a \mathscr{S}$.

Como veremos no decorrer deste capítulo, pares admissíveis são importantes na teoria de equações diferenciais impulsivas, pois, através da admissibilidade de um par de espaços de funções para a EDI não-homogênea (5.1), (5.2) eles definem o caminho dicotômico da EDI homogênea associada, isto é, a EDI (3.1), (3.2).

\subsection{Dicotomia Ordinária e Admissibilidade}

Nesta seção, relacionamos a existência de dicotomia ordinária para a EDI homogênea (3.1), (3.2) e a existência de soluções limitadas da EDI não-homogênea (5.1), (5.2).

No texto original [1], Teorema 7.1, o autor apresenta o teorema abaixo com a hipótese adicional de que

Existem um número $l>0$ e um inteiro positivo $\lambda$ tais que cada intervalo em $\mathbb{R}_{+}$com comprimento 1 não contém mais que $\lambda$ pontos da seqüencia $\left\{t_{n}\right\}_{n \in \mathbb{N}}$.

Entretanto tal hipótese não foi necessária conforme podemos observar em sua demonstração.

Teorema 5.1 Suponhamos que a EDI (3.1), (3.2) sobre $\mathbb{R}_{+}$tenha uma dicotomia ordinária e que $\operatorname{det} Q_{n} \neq 0$, para qualquer $n \in \mathbb{N}$. Entāo, para toda função $f \in \mathscr{L}_{1}\left(\mathbb{R}_{1}, X\right)$ e toda sequiência he $\ell_{1}(\mathbb{N})$, existirá uma solução $x(t)$ da equação não-homogênea $(5.1),(5.2)$ que será limitada em $\mathbb{R}_{+}$, ou seja, existirá uma solução $x \in \mathscr{B}\left(\mathbb{R}_{+}, X\right)$ da equação não-homogênea (5.1), (5.2). 
Demonstração: Consideremos a equação $x(t)$ definida pela fórmula

$$
\begin{aligned}
x(t) & =\int_{0}^{t} U(t) P U^{1}(\tau) f(\tau) d \tau-\int_{t}^{\infty} U(t)(I-P) U^{1}(\tau) f(\tau) d \tau+ \\
& +\sum_{t_{j}<t} U(t) P U^{-1}\left(t_{j}^{+}\right) h_{j}-\sum_{t_{j} \geq t} U(t)(I-P) U^{-1}\left(t_{j}^{+}\right) h_{j} .
\end{aligned}
$$

Provemos, inicialmente, que $x(t)$ é uma solução da FDI (5.1), (5.2). Para isto, utilizaremos as igualdades

$$
\frac{d}{d t} U(t, \tau)=A(t) U(t, \tau), \quad \frac{d}{d \tau} U(t, \tau)=-U(t, \tau) A(\tau)
$$

e

$$
U\left(t_{n}^{+}, \tau\right)=Q_{n} U\left(t_{n}, \tau\right), \quad 0 \leq \tau, t_{n}<\infty, n \in \mathbb{N}
$$

$(\operatorname{veja}(2.6))$

Observemos que, pela definição de $x(t)$ e pelos fatos de $A: \mathbb{R}_{+} \rightarrow L(X)$ ser uma função contínua por partes, com descontinuidade de primeira espécie em $t=t_{n}$, contínua à esquerda em $t=t_{n}, n \in \mathbb{N}$, e de $f: \mathbb{R}_{+} \rightarrow X$ ser uma função contínua por partes, com descontinuidade de primeira espécie em $t=t_{n}$ e contínua à esquerda em $t=t_{n}, n \in \mathbb{N}$, concluímos que $x(t)$ é contínua para $t \neq t_{n}$ e que o valor limite $x\left(t_{n}^{+}\right)$existe, para qualquer $n \in \mathbb{N}$. Diferenciando $x(t)$ para $t \neq t_{n}$, obtemos

$$
\begin{aligned}
\frac{d x(t)}{d t} & =U(t) \frac{d}{d t}\left(\int_{0}^{t} P U^{-1}(\tau) f(\tau) d \tau\right)+\frac{d U(t)}{d t} \int_{0}^{t} P U^{-1}(\tau) f(\tau) d \tau- \\
& -U(t) \frac{d}{d t}\left(\int_{t}^{\infty} U(t)(I-P) U^{-1}(\tau) f(\tau) d \tau\right)-\frac{d U(t)}{d t} \int_{t}^{\infty} U(t)(I-P) U^{-1}(\tau) f(\tau) d \tau+ \\
& +\sum_{t_{j}<t} \frac{d U(t)}{d t} P U^{-1}\left(t_{j}^{+}\right) h_{j}-\sum_{t_{j}>t} \frac{d U(t)}{d t} U(t)(I-P) U^{-1}\left(t_{j}^{+}\right) h_{j}= \\
& =U(t) P U^{-1}(t) f(t)-U(t)(I-P) U^{-1}(t) f(t)+ \\
& +\int_{0}^{l} A(t) U(t) P U^{-1}(\tau) f(\tau) d \tau-\int_{t}^{\infty} A(t) U(t)(I-P) U^{-1}(\tau) f(\tau) d \tau+ \\
& +\sum_{l_{j}<t} A(t) U(t) P U^{-1}\left(t_{j}^{+}\right) h_{j} \cdot \sum_{t_{j}>t} A(t) U(t)(I-P) U^{-1}\left(t_{j}^{-}\right) h_{j}= \\
& =f(t)+A(t) x(t) .
\end{aligned}
$$

Além disso, 


$$
\begin{aligned}
x\left(t_{n}^{+}\right)= & \int_{0}^{t_{n}^{+}} U\left(t_{n}^{+}\right) P U^{-1}(\tau) f(\tau) d \tau-\int_{t_{n}^{+}}^{\infty} U\left(t_{n}^{+}\right)(I-P) U^{-1}(\tau) f(\tau) d \tau+ \\
& +\sum_{t_{j}<t_{n}^{+}} U\left(t_{n}^{-}\right) P U^{-1}\left(t_{j}^{+}\right) h_{j}-\sum_{j_{j} \geq t_{n}^{+}} U\left(t_{n}^{-}\right)(I-P) U^{-1}\left(t_{j}^{+}\right) h_{j}= \\
& =\int_{0}^{t_{n}^{+}} Q_{n} U\left(t_{n}\right) P U^{-1}(\tau) f(\tau) d \tau-\int_{t_{n}^{+}}^{\infty} Q_{n} U\left(t_{n}\right)(I-P) U^{-1}(\tau) f(\tau) d \tau+ \\
& +\sum_{t_{j}<t_{n}^{+}} Q_{n} U\left(t_{n}\right) P U^{-1}\left(t_{j}^{+}\right) h_{j}-\sum_{t_{j} \geq t_{n}^{+}} Q_{n} U\left(t_{n}\right)(I-P) U^{-1}\left(t_{j}^{-}\right) h_{j}= \\
& =Q_{n} \int_{0}^{t_{n}} U\left(t_{n}\right) P U^{-1}(\tau) f(\tau) d \tau-Q_{n} \int_{t_{n}}^{\infty} U\left(t_{n}\right)(I-P) U^{-1}(\tau) f(\tau) d \tau+ \\
& +Q_{n} \sum_{t_{j}<t_{n}} U\left(t_{n}\right) P U^{-1}\left(t_{j}^{+}\right) h_{j}+Q_{n} U\left(t_{n}\right) P U^{-1}\left(t_{n}^{+}\right) h_{n}- \\
& -Q_{n} \sum_{i_{j} \geq t_{n}} U\left(t_{n}\right)(I-P) U^{-1}\left(t_{j}^{+}\right) h_{j}+Q_{n} U\left(t_{n}\right)(I-P) U^{-1}\left(t_{n}^{+}\right) h_{n}
\end{aligned}
$$

e, portanto,

$$
x\left(t_{n}^{+}\right)=Q_{n} x\left(t_{n}\right)+h_{n},
$$

para qualquer $n \in \mathbb{N}$.

Agora, estimaremos o módulo de $x(t)$. Temos

$$
\begin{aligned}
|x(t)| & \leq \int_{0}^{t}\left|U(t) P U^{-1}(\tau)\right||f(\tau)| d \tau+\int_{t}^{\infty}\left|U(t)(I-P) U^{-1}(\tau)\right||f(\tau)| d \tau+ \\
& +\sum_{t_{j}<t}\left|U(t) P U^{-1}\left(t_{j}^{+}\right)\right|\left|h_{j}\right|+\sum_{t_{j} \geq t}\left|U(t)(I-P) U^{-1}\left(t_{j}^{+}\right)\right|\left|h_{j}\right| .
\end{aligned}
$$

Como a EDI (3.1), (3.2) tem uma dicotomia ordinária, segue da Definiçāo 3.5 que existem uma constante $K>0$ e uma projeção $P: X \rightarrow X$ tais que

$$
\left|U(t) P U^{-1}(s)\right| \leq K, \quad \text { para } s \leq t
$$

e

$$
U(t)(I-P) U^{-1}(s) \mid \leq K, \quad \text { para } s \geq t .
$$

Assim, 


$$
\begin{aligned}
|x(t)| & \leq K \int_{0}^{t}|f(\tau)| d \tau+K \int_{t}^{\infty}|f(\tau)| d \tau+K \sum_{t_{j}<t}\left|h_{j}\right|+K \sum_{t_{j} \geq t}\left|h_{j}\right| \\
& =K \int_{0}^{\infty}|f(\tau)| d \tau+K \sum_{j=1}^{\infty}\left|h_{j}\right|
\end{aligned}
$$

e, como $f \in \mathscr{L}_{1}\left(\mathbb{R}_{+}, X\right)$ e $h \in \ell_{1}(\mathbb{N})$, segue que $|x(t)|<\infty$. Portanto o teorema está provado.

No restante deste capítulo, vamos considerar que $X_{1}$ é o espaço dos $x(\xi) \in X$ para os quais a função $U(t) x(\xi), t \geq \xi$, é limitada c que $\mathscr{R}^{0}\left(\mathbb{R}_{+}, X\right)$ é o subespaço de $\mathscr{R}\left(\mathbb{R}_{+}, X\right)$ que consiste das funções que satisfazem a condição

$$
x\left(t_{n}^{+}\right)-Q_{n} x\left(t_{n}\right)=0
$$

ou seja, $h_{n}=0, n \in \mathbb{N}$.

Como anteriormente, também definimos o seguinte espaço

$$
\mathscr{B}^{0}(\infty):=\left\{f \in \mathscr{B}^{0}\left(\mathbb{R}_{+}, X\right): \lim _{t \rightarrow+\infty} f(t) \text { existe }\right\}
$$

isto é, $\mathscr{B}^{0}(\infty)$ é o espaço de funções de $\mathscr{B}^{0}\left(\mathbb{R}_{-}, X\right)$ com limite no infinito. Nos espaços $\mathscr{B}^{0}\left(\mathbb{R}_{+}, X\right)$ e $\mathscr{B ^ { 0 }}(\infty)$ consideremos a norma induzida de $\mathscr{B}\left(\mathbb{R}_{+}, X\right)$. Além disso, se $A: \mathbb{R}_{+} \rightarrow L(X)$ for tal que $A(t)$ é invertível para cada $t \in \mathbb{R}_{+}$, isto é, existe $A^{-1}(t): X \rightarrow X$, para cada $t \in \mathbb{R}_{+}$, então definiremos

$$
\mathscr{B}_{A}^{0}(\infty):=\left\{f \in \mathscr{B}^{0}\left(\mathbb{R}_{+}, X\right): A^{-1} f \in \mathscr{B}^{0}(\infty)\right\}
$$

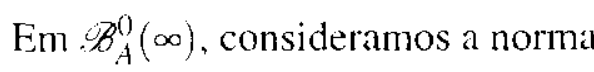

$$
\left\|\left.f\right|_{i A}=\right\| A f \|_{\infty}
$$

Se $A$ for limitada, então $\mathscr{B}_{A}^{0}(\infty)$ será um espaço de Banach.

Lema 5.1 /[1], Lema 7.1/ Suponhamos que $\mathscr{F}\left(\mathbb{R}_{+}, X\right)$ seja um espaço de Banach arbitrário de funçães $f: \mathbb{R}_{+} \rightarrow X$ e, para qualquer funçāo $f \in \mathscr{F}\left(\mathbb{R}_{+}, X\right)$, a equação não-homogênea (5.1), (5.2) tenha pelo menos uma solução $x \in \mathscr{B}^{0}\left(\mathbb{R}_{+}, X\right)$ limitada em $\mathbb{R}_{-}$. Entāo, para toda função $f \in \mathscr{F}\left(\mathbb{R}_{+}, X\right)$, existirá uma única soluşão limitada em $\mathbb{R}_{+}$iniciando em $X_{2}$, onde $X_{2}$ é qualquer 
complemento de $X_{1}$ em $X$, satisfazendo a estimativa

$$
\|x\|_{\infty} \leq M\|f\|_{\mathscr{F}},
$$

onde $M$ é uma constante independente de f e dependente, somente, do espaço $\mathscr{F}\left(\mathbb{R}_{+}, X\right)$.

Demonstração: Não é difícil ver que, se $x_{1}(t)$ e $x_{2}(t)$ forem duas soluçõcs da EDI não-homogênea (5.1), (5.2), então sua diferença, $z(t)=x_{1}(t)-x_{2}(t)$, será uma solução da EDI homogênea (3.1), (3.2). Se as soluções $x_{1}(t)$ e $x_{2}(t)$ forem limitadas, então $z(t)$ também será limitada. Portanto $z(0) \in X_{1}$. Se $x(t)$ for uma solução de $(5.1),(5.2) \mathrm{em} \mathscr{B}^{0}(\mathbb{R} \ldots, X)$, então $\tilde{x}(t)=x(t)-U(t) P x(0)$ também será uma solução de (5.1), (5.2) em $\check{b}^{0}\left(\mathbb{R}_{+}, X\right)$, com valor inicial $\tilde{x}(0)=(I-P) x(0) \in X_{2}$. De fato. Temos

$$
\begin{gathered}
\tilde{x}\left(t_{n}^{+}\right)-Q_{n} \tilde{x}\left(t_{n}\right)=x\left(t_{n}^{+}\right)-U\left(t_{n}^{+}\right) P x(0)-Q_{n}\left[x\left(t_{n}\right)-U\left(t_{n}\right) P x(0)\right]= \\
=Q_{n} x\left(t_{n}\right)-Q_{n} U\left(t_{n}\right) P x(0)-Q_{n} x\left(t_{n}\right)-Q_{n} U\left(t_{n}\right) P x(0)=0 .
\end{gathered}
$$

Portanto $\tilde{x} \in \mathscr{B}^{0}\left(\mathbb{R}_{-}, X\right)$ e como

$$
\tilde{x}(0)=x(0)-U(0) P x(0)=x(0)-P x(0)-(I-P) x(0) \in X_{2},
$$

pelas condições do Lema 5.1, segue que, para $f \in \mathscr{F}\left(\mathbb{R}_{+}, X\right)$, a EDI não-homogênea (5.1), (5.2) tem uma única solução $\tilde{x}(t) \in \mathscr{B}^{0}\left(\mathbb{R}_{+}, X\right)$ satisfazendo a igualdade $P \tilde{x}(0)=0$. Assim, definimos o operador $\tilde{K}: \mathscr{F}\left(\mathbb{R}_{+}, X\right) \rightarrow \mathscr{g}^{0}\left(\mathbb{R}_{+}, X\right)$ pela associação de cada elemento $f \in \mathscr{F}\left(\mathbb{R}_{+}, X\right)$ a uma solução da EDI não-homogênea (5.1), (5.2).

Provemos que $\tilde{K}$ tem gráfico fechado. Suponhamos que $f_{m} \rightarrow f \mathrm{~cm} \mathscr{F}\left(\mathbb{R}_{+}, X\right)$ e que $y_{m}=\widetilde{K} f_{m} \rightarrow y$. Então

$$
y(0)=\lim _{m \rightarrow \infty} y_{m}(0) \in X_{2}
$$

$e$, para qualquer $t$ fixado, temos

$$
\int_{0}^{t} f(s) d s=\lim _{m \rightarrow \infty} \int_{0}^{t} f_{m}(s) d s .
$$

Portanto, 


$$
\begin{aligned}
y(t)-y(0) & =\int_{0}^{t} y^{\prime}(s) d s= \\
& =\int_{0}^{t_{1}} y^{\prime}(s) d s+\sum_{i=1}^{n-1} \int_{t_{i}}^{t_{i+1}} y^{\prime}(s) d s+\int_{t_{n}}^{t} y^{\prime}(s) d s= \\
& =\int_{0}^{t_{1}} \lim _{m \rightarrow \infty} y_{m}^{\prime}(s) d s+\sum_{i=1}^{n-1} \int_{t_{i}}^{t_{t+1}} \lim _{m \rightarrow \infty} y_{m}^{\prime}(s) d s+\int_{t_{n}}^{t_{m \rightarrow \infty}} \lim _{m \rightarrow \infty} y_{m}^{\prime}(s) d s \\
& =\lim _{m \rightarrow \infty} \int_{0}^{t_{1}} y_{m}^{\prime}(s) d s+\sum_{i-1}^{n-1} \lim _{m-\infty} \int_{t_{i}}^{t_{i+1}} y_{m}^{\prime}(s) d s+\lim _{m \cdot>\infty} \int_{t_{n}}^{t} y_{m}^{\prime}(s) d s= \\
& =\lim _{m \rightarrow \infty} \int_{0}^{t_{1}}\left[A(s) y_{m}(s)+f_{m}(s)\right] d s+\sum_{i=1}^{n-1} \lim _{m \rightarrow \infty} \int_{t_{i}}^{t_{i+1}}\left[A(s) y_{m}(s)+f_{m}(s)^{\prime} d s+\right. \\
& +\lim _{m \rightarrow \infty} \int_{t_{n}}^{t}\left[A(s) y_{m}(s)+f_{m}(s)\right] d s= \\
& =\int_{0}^{t_{1}}[A(s) y(s)+f(s)] d s+\sum_{i=1}^{n-1} \int_{t_{i}}^{t_{i+1}}[A(s) y(s)+f(s)] d s+ \\
& +\int_{t_{n}}^{t}[A(s) y(s)+f(s)] d s \\
& =\int_{0}^{t}[A(s) y(s)+f(s)] d s .
\end{aligned}
$$

E, como

$$
y\left(t_{n}^{+}\right)=\lim _{m \rightarrow \infty} y_{m}\left(t_{n}^{+}\right)=\lim _{m \rightarrow \infty} Q_{n} y_{m}\left(t_{n}\right)=Q_{n} y\left(t_{n}\right),
$$

segue que $y(t)$ é uma solução da equação (3.1), (3.2). Portanto, pelo Teorema do Gráfico Fechado de Banach, o operador $\widetilde{K}$ é limilado, isto é, existe uma constante positiva $M$ tal que

$$
\|\tilde{K} f\|_{\infty} \leq M\|f\|_{\tilde{y}}
$$

e a prova está completa.

Agora, para continuarmos, introduziremos a seguinte função de Green

$$
G(t, s)= \begin{cases}U(t) P U(s), & s \leq t \\ -U(t)(I-P) U(s), & s>t\end{cases}
$$

que será utilizada nas demonstrações dos próximos teoremas. 
O resultado a seguir dá uma recíproca do Teorema 5.1 para o caso em que $h=0$. Cabe observarmos que, para este teorema, damos uma prova alternativa pois na demonstração dada em [1] alguns pontos não ficaram suficientemente claros.

Teorema 5.2 [/1], Teorema 7.2/ Suponhamos que a EDI näo-homogênea (5.1), (5.2) tenha uma única solução limitada na semi-reta $\mathbb{R}_{+}$, para qualquer função $f \in \mathscr{L}_{1}(\mathbb{R}, X)$ e para $h=0$. Suponhamos, ainda, que det $Q_{n} \neq 0$, para qualquer $n \in \mathbb{N}$. Então a EDI homogênea (3.1), (3.2) terá uma dicotomia ordinária.

Demonstração: Para provarmos que a EDI homogênea (3.1), (3.2) tem uma dicotomia ordinária, é suficiente mostrarmos que, para $0 \leq \tau, t<\infty$, temos

$$
\|G(t, \tau)\| \leq K
$$

onde $K$ é uma constante. Para isto, sejam $a, s \in \mathbb{R}$ arbitrários e consideremos a função $f_{a}$ definida por

$$
f_{a}(t)= \begin{cases}z, & t \in[s-a, s], \\ 0, & t \notin[s-a, s],\end{cases}
$$

onde $s \geq a>0$ e $z \in X, \operatorname{com}|z|=1$. Vemos que $f_{a} \in \mathscr{L}_{1}\left(\mathbb{R}_{+}, X\right)$.

Definamos, agora, a função $y_{a}(t)$ por

$$
y_{a}(t)=\int_{s-a}^{a} G(t, \tau) z d \tau
$$

Para $t \geq a$, temos $y_{a}(t)=U(t) \xi$, onde

$$
\xi=\int_{s \cdots a}^{s} P U^{\cdots 1}(\tau) z d \tau
$$

isto é, a função $y_{a}(t)$ é limitada.

Para $0 \leq t \leq a$, a função $y_{a}(t)$ também é limitada, pois ela é contínua num intervalo compacto. Portanto $y_{a}(t)$ é limitada na semi-reta $\mathbb{R}_{+}$, e mais, temos

$$
y_{a}(0)=\int_{s-a}^{s} G(0, \tau) z d \tau=-(I-P) \int_{s-a}^{s} U^{-1}(\tau) z d \tau
$$


Logo $y_{a}(0) \in X_{2}$. Assim, pelo Lema 5.1, existe uma constante $M>0$ tal que

$$
\left|y_{a}\right|_{\infty} \leq M\left\|f_{a}\right\|_{1}=M \int_{s-a}^{s}|z| d \tau=M a|z|=M a,
$$

isto é,

$$
\left|\int_{s-a}^{a} G(t, \tau) z d \tau\right| \leq M a
$$

e, portanto,

$$
\left|\frac{1}{a} \int_{s-a}^{a} G(t, \tau) z d \tau\right| \leq M
$$

Daí, fazendo $a$ tender a zero, obtemos

$$
|G(t, s) z| \leq M
$$

onde $|z|=1 \mathrm{e}$, portanto,

$$
|| G(t, s) \| \leq M
$$

para $0 \leq s ; t<\infty$. Logo a EDI (3.1), (3.2) tem uma dicotomia ordinária.

\subsection{Dicotomia Exponencial e Admissiblidade}

Nesta seção, discutiremos as relações entre a existência de dicotomia exponencial para a EDI (3.1), (3.2) sobre $\mathbb{R}_{+}$e a existência de soluçōes limitadas da equação não-homogênea (5.1), (5.2). Seja $\ell_{1}(X)$ o espaço de todas as seqüências $\left\{a_{n}\right\}_{n \in \mathbb{N}} \mathrm{em} X$ tal que $\sum_{n \in \mathbb{N}}\left|a_{n}\right|<\infty$.

Teorema 5.3 [/1], Teorema 7.3] Suponhamos que

(i) Existam un numero $l>0$ e um inteiro positivo $\lambda$ tais que cada internalo em $\mathbb{R}_{+}$com comprimento I não contenha mais que $\lambda$ pontos da seqüência $\left\{t_{n}\right\}_{n \in \mathbb{N}}$;

(ii) A EDI (5.1), (5.2) tenha uma dicotomia exponencial;

(iii) $\operatorname{det} Q_{n} \neq 0$, para todo $n \in \mathbb{N}$. 
Então,

1. Para toda função $f \in \mathscr{P}\left(\mathbb{R}_{+}, X\right)$ e toda seqüencia $\left\{h_{n}\right\}_{n \in \mathbb{N}} \in \ell_{1}(X)$, existirá pelo menos uma solução limitada da EDI não-homogênea (5.1), (5.2) sobre $\mathbb{R}_{+}$.

2. O operador linear $L: \not B\left(\mathbb{R}_{+}, x\right) \rightarrow \mathscr{B}\left(\mathbb{R}_{+}, X\right) \times \ell_{1}(X)$ definido por

$$
L x(t)=\left(\frac{d x}{d t}-A(t) x, x\left(t_{n}^{+}\right)-Q_{n} x\left(t_{n}\right)\right)
$$

admitirá um operador inverso contínuo à direita.

Demonstração: Primeiramente, mostraremos que o operador $\tilde{K}: \mathscr{B}\left(\mathbb{R}_{+}, X\right) \times \ell_{1}(X) \rightarrow \mathscr{B}\left(\mathbb{R}_{\mathrm{T}}, X\right)$ definido por

$$
\begin{aligned}
\tilde{K}(f, h)(t) & =\int_{0}^{t} U(t) P U^{-1}(\tau) f(\tau) d \tau-\int_{t}^{\infty} U(t)(I-P) U^{\cdots 1}(\tau) f(\tau) d \tau+ \\
& +\sum_{t_{j}<t} U(t) P U^{-1}\left(t_{j}^{+}\right) h_{j}-\sum_{t_{l} \geq t} U(t)(I-P) U^{-1}\left(t_{j}^{+}\right) h_{j}
\end{aligned}
$$

é contínuo e satisfaz

$$
L \widetilde{K}=I
$$

onde $I$ é o operador identidade no espaço $\mathscr{B}\left(\mathbb{R}_{-i}, X\right)$. De fato. Notemos que

$$
\begin{aligned}
\left|\int_{0}^{t} U(t) P U^{-1}(\tau) f(\tau) d \tau\right| & \leq \int_{0}^{t}\left|U(t) P U^{-1}(\tau) \| f(\tau)\right| d \tau \leq \\
& \leq K e^{-\alpha t} \int_{0}^{t} e^{\alpha \tau} d \tau\|f\|_{\infty} \leq \frac{K}{\alpha}\|f\|_{\infty}
\end{aligned}
$$

e que

$$
\begin{aligned}
\left|\int_{t}^{\infty} U(t)(I-P) U^{-1}(\tau) f(\tau) d \tau\right| & \leq \int_{t}^{\infty}\left|U(t)(I-P) U^{-1}(\tau)\right| f(\tau) \mid d \tau \leq \\
& \leq K e^{\alpha \ell} \int_{t}^{\infty} e^{-\alpha \tau} d \tau\|f\|_{\infty} \leq \frac{K}{\alpha} \mid: f \|_{\infty} .
\end{aligned}
$$

Analogamente, temos 


$$
\begin{aligned}
\left|\sum_{t_{j}<t} U(t) P U^{-1}\left(t_{j}^{+}\right) h_{j}\right| & \leq \sum_{t_{j}<t}\left|U(t) P U^{-1}\left(t_{j}^{+}\right) \| h_{j}\right| \leq \\
& \leq K\left(\sum_{l_{j}<l} e^{\alpha\left(t_{j}-t\right)}\right)\|h\|_{1} \leq \frac{K \lambda}{1-e^{-\alpha l}}\|h\|_{1}
\end{aligned}
$$

$$
\begin{aligned}
\left|\sum_{t_{j} \geq t} U(t)(I-P) U^{-1}\left(t_{j}^{+}\right) h_{j}\right| & \leq \sum_{t_{j} \geq t}\left|U(t)(I-P) U^{-1}\left(t_{j}^{+}\right) \| h_{j}\right| \leq \\
& \leq K\left(\sum_{l_{j} \geq t} e^{\alpha\left(t-t_{j}\right)}\right)\left\|h_{1} \leq \frac{K \lambda}{1-e^{-\alpha l}}\right\| h \|_{1} .
\end{aligned}
$$

De (5.9) até (5.12), segue que a função $\tilde{K}(f, h)$ é limitada e satisfaz

$$
\tilde{K}(f, h)\left\|_{\infty} \leq \frac{2 K}{\alpha}\right\| f\left\|_{\infty}+\frac{2 K \lambda}{1-e^{-\alpha l}}\right\| h \|_{1} .
$$

Pela definição de $\tilde{K}(f, h)$ e como $f \in \mathscr{B}\left(\mathbb{R}_{+}, X\right)$ e $h \in \ell_{1}(X)$, concluímos que $\tilde{K}(f, h)$ é contínua em $t \neq t_{n}$ e que existe o valor limite $\tilde{K}(f, h)\left(t_{n}^{+}\right)$, para qualquer $n \in \mathbb{N}$.

Ainda resta verificarmos (5.8).

Diferenciando (5.7) para $t \neq t_{n}$ e utilizando (5.3), (5.4) e as igualdades

$$
U(t, \tau)=U^{-1}(\tau, t) \quad \text { e } \quad U(t, \tau)=U(t, s) U(s, \tau)
$$

onde $0 \leq \tau, s, t<\infty$ (veja (2.6)), obtemos

$$
\begin{aligned}
\frac{\tilde{K}(f, h)(t)}{d t} & =U(t) P U^{-1}(t) f(t)+U(t)(I-P) U^{-1}(t) f(t)+ \\
& +\int_{0}^{t} A(t) U(t) P U^{-1}(\tau) f(\tau) d \tau-\int_{t}^{\infty} A(t) U(t)(I-P) U^{-1}(\tau) f(\tau) d \tau+ \\
& +\sum_{t_{j}<t} A(t) U(t) P U^{-1}\left(t_{j}\right) h_{j}-\sum_{t_{j} \geq t} A(t) U(t)(I-P) U^{-1}\left(t_{j}^{+}\right) h_{j}= \\
& =f(t)+A(t)(\tilde{K}(f, h)(t)) .
\end{aligned}
$$


Além disso, para cada $n \in \mathbb{N}$, temos

$$
\begin{aligned}
\tilde{K}(f, h)\left(t_{n}^{+}\right) & =\int_{0}^{t_{n}} U\left(t_{n}^{\prime}\right) P U^{-1}(\tau) f(\tau) d \tau-\int_{t_{n}}^{\infty} U\left(t_{n}^{+}\right)(I-P) U^{-1}(\tau) f(\tau) d \tau+ \\
& +\sum_{t_{j}<t_{n}^{+}} U\left(t_{n}^{+}\right) P U^{1}\left(t_{j}^{+}\right) h_{j}-\sum_{t_{j} \geq t_{n}^{\prime}} U\left(t_{n}^{+}\right)(I-P) U^{-1}\left(t_{j}^{+}\right) h_{j}
\end{aligned}
$$

Daí, utilizando (5.4), obtemos

$$
\begin{aligned}
\tilde{K}(f, h)\left(t_{n}^{\prime}\right) & =Q_{n} \int_{0}^{t_{n}} U\left(t_{n}\right) P U^{-1}(\tau) f(\tau) d \tau-Q_{n} \int_{t_{n}}^{\infty} U\left(t_{n}\right)(I-P) U^{-1}(\tau) f(\tau) d \tau+ \\
& +Q_{n} \sum_{l_{i}<t_{n}^{+}} U\left(t_{n}\right) P U^{-1}\left(t_{j}^{+}\right) h_{j}-Q_{n} \sum_{l_{j} \geq t_{n}^{\prime}} U\left(t_{n}\right)(I-P) U^{-1}\left(t_{j}^{-}\right) h_{j}+ \\
& +U\left(t_{n}^{+}\right) P U^{-1}\left(t_{n}^{+}\right) h_{n}+U\left(t_{n}^{+}\right)(I-P) U^{-1}\left(t_{n}^{+}\right) h_{n}= \\
& =Q_{n} \tilde{K}(f, h)\left(t_{n}\right)+h_{n} .
\end{aligned}
$$

Assim,

$$
\begin{aligned}
& L(\tilde{K}(f, h)(t))= \\
& =\left(\frac{d \tilde{K}(f, h)(t)}{d t}-A(t)(\tilde{K}(f: h)(t)),\left(\tilde{K}(f, h)\left(t_{n}^{-}\right)\right)-Q_{n}\left(\tilde{K}(f, h)\left(t_{n}\right)\right)\right)= \\
& =\left(f(t)+A(t)(\tilde{K}(f, h)(t))-A(t)(\tilde{K}(f, h)(t)), Q_{n}\left(\tilde{K}(f, h)\left(t_{n}\right)\right)+h_{n}-Q_{n}\left(\tilde{K}(f, h)\left(t_{n}\right)\right)\right)= \\
& =\left(f(t), h_{n}\right)=(f, h)(t)
\end{aligned}
$$

e, deste modo, terminamos a demonstração.

Observação 5.1 Podemos substituir a condição

$$
\sup _{t \in \mathbb{R} .+} i f(t) \|<\infty
$$

do Teorema 5.3 pela condição mais fraca

$$
\sup _{t \in \mathbb{E}_{+}} \int_{i}^{t+1}|f(\tau)| d \tau<\infty
$$

Observação 5.2 O Teorema 5.3 ainda continua válido sem a condição (i), se considerarmos a equação não-homogênea com $h=0$. Neste caso, os valores do operador definido por (5.7) ficam 
no subespaço $\mathscr{B}^{0}(\mathbb{R} . X)$ (veja o Lema 5.1$)$.

Teorema 5.4 // I], Terrema 7.4/ Suponhamos que a EDI (3.1), (3.2) sobre $\mathbb{R}_{+}$satisfaça as condiçōes

(i) $g(X)<\infty e g^{\prime}(X)>-\infty$;

(ii) Para toda função $f \in \mathscr{B}\left(\mathbb{R}_{+}, X\right)$, a EDI não-homogênea (5.1), (5.2), com $h=0$, tem pelo menos uma solução iniciando no espaço $\mathscr{B}\left(\mathbb{R}_{+}, X\right)$;

(iii) $\operatorname{det} Q_{n} \neq 0$, para $n \in \mathbb{N}$.

Então, a EDI (3.1), (3.2) terá uma dicotomia exponencial.

Demonstração: Seja $X_{1}$ o subespaço de $X$ consistindo de todo $\xi$ tal que

$$
\sup _{0 \leq \leq<\infty}|U(t) \xi|<\infty
$$

Denotemos por $P$ a projeção de $X$ sobre $X_{1}$, isto é, $X_{1}=P X$.

Observemos que se $x_{3}(t)$ e $x_{2}(t)$ forem duas soluções da EDI não-homogênea (5.1), (5.2), então sua diferença, $z(t)=x_{1}(t)-x_{2}(t)$, será uma solução da EDI homogênea (3.1), (3.2). E, se as soluções $x_{1}(t)$ e $x_{2}(t)$ forem limitadas, então $z(t)$ também será limitada. Portanto $z(0) \in$ $X_{1}$. Se $x(t)$ for uma solução de $(5.1),(5.2) \mathrm{em} \mathscr{R}^{0}\left(\mathbb{R}_{+}, X\right)$, segue que a equação $x(t)=x(t)-$ $U(t) P x(0)$ também será uma solução de (5.1), (5.2) pertencente a $\mathscr{B}^{0}\left(\mathbb{B}_{+}, X\right)$, com valor inicial $\tilde{x}(0)=(I-P) x(0) \in \operatorname{Ker} P$.

Por hipótese, para cada $f \in \mathscr{B}\left(\mathbb{R}_{+}, X\right)$, a EDI não-homogênea (5.1), (5.2), com $h=0$, tem uma única solução $\tilde{x}(t) \in \mathscr{B}^{0}\left(\mathbb{X}_{+}, X\right)$ tal que $P \tilde{x}(0)=0$. Desta forma, definimos o operador $\tilde{K}$ como sendo a função de $\mathscr{B}\left(\mathbb{R}_{+}, X\right) \mathrm{em}: \mathscr{b}^{0}\left(\mathbb{R}_{+}, X\right)$ que associa a cada elemento $f \in \mathscr{B}\left(\mathbb{R}_{+}, X\right)$ uma solução da EDI não-homogênea (5.1), (5.2), $\operatorname{com} h=0$. Pelo Teorema do Gráfico Fechado de Banach, este operador é contínuo, isto é, existe um número $M$ lal que

$$
\|\tilde{K} f\|_{\infty} \leq M\left\|f^{\prime}\right\|_{\infty} .
$$


Agora, provemos que

$$
\left\|U(t) P U^{-1}(s)\right\| \leq K e^{-\alpha(t \cdot s)}, \quad 0 \leq s \leq t<\infty
$$

eque

$$
\|\left. U(t)(I-P) U^{-1}(s)\right|_{i} \leq K e^{-\alpha(t-s)}, \quad \leq s \leq t<\infty
$$

onde $K$ e $\alpha$ são constantes positivas.

Seja $x(t)$ uma solução limitada da EDI (3.1), (3.2) sobre $\mathbb{R}_{+}$, com valor inicial $x(0) \in X_{1}$. Para cada $a \in \mathbb{R}$, definimos

$$
y_{a}(t)=\left[\left.\int_{0}^{t} \chi_{a}(\tau) ! x(\tau)\right|^{-1} d \tau\right] x(t)
$$

onde

$$
\chi_{a}(t)= \begin{cases}1, & 0 \leq t<\tau+a, \\ 1-(t-\tau-a), & \tau+a<t \leq \tau+a+1, \\ 0, & \tau+a+1 \leq t<\infty .\end{cases}
$$

Podemos ver que a funçăo $y_{a}(t)$ é uma solução da EDI (5.1), (5.2) com $h=0$. Seja $f_{a}$ dada por

$$
f_{a}(t)=\chi_{a}(t)|x(t)|^{-1} x(t)
$$

Então $f_{a} \in \mathscr{R}\left(\mathbb{R}_{+}, X\right) \propto\left\|f_{a}\right\|_{\infty}=1$. Logo $(5.13)$ implica que

$$
\left\|y_{a}(t)\right\| \leq K,
$$

Em outras palavras,

$$
\left[\int_{0}^{t} \chi_{a}(\tau)|x(\tau)|^{-1} d \tau\right]|x(t)| \leq K
$$

Daí, fazendo $a \rightarrow+\infty$, obtemos

$$
\left[\int_{0}^{t} \mid x(\tau)^{\prime-1} d \tau\right], x(t) \mid \leq K
$$

Como $g(X)<\infty$ e $g^{\prime}(X)>-\infty$, deduzimos que existem constantes positivas $\bar{M}$ e $\bar{N}$ tais que 
qualquer solução da EDI (3.1), (3.2) satisfaz

$$
x(t)|\leq| x(s) \mid \tilde{M} e^{\tilde{N}(t-s)}, \quad 0 \leq t, s<\infty .
$$

Seja $t-s \geq 1$. Então existe $\tau \geq 1$ tal que $t=s+\tau$ e vale

$$
|x(t)| \int_{s}^{t}\left|x(u)^{\mid-1} d u \leq\right| x(t)\left|\int_{0}^{t} x(u)\right|^{-1} d u \leq K
$$

Introduziremos, agora, a seguinte função

$$
\varphi(t)=\int_{s}^{t}|x(u)|^{-1} d u
$$

De (5.17), obtemos

$$
\frac{\frac{d \varphi(s+u)}{d t}}{\varphi(s+u)} \geq \frac{1}{K}
$$

que, após integrarmos com respeito a $u$ sobre $[1, \tau]$, nos dará

$$
\varphi(t)=\varphi(s+\tau) \geq \varphi(s+1) e^{(\tau+1) / K}
$$

Agora, por (5.16), segue que, para $u \geq s$, temos

$$
|x(u)|^{-1} \geq|x(s)|^{-1} \check{M}^{-1} e^{-\tilde{N}(u \cdots s)},
$$

o que implica que

$$
\begin{aligned}
\varphi(s+1) & -\int_{s}^{s+1}\left|x(u)_{i}{ }^{1} d u \geq \int_{s}^{s+1}\right| x(s)_{i}{ }^{-1} \tilde{M}^{-1} e^{-\tilde{N}(u-s)} d u- \\
& =|x(s)|{ }^{1} \tilde{M}^{-1} e^{\tilde{N} s} \int_{s}^{s+1} e^{\tilde{N} u} d u=|x(s)|^{\cdot 1} \tilde{M}^{1}\left(1-e^{\tilde{N}}\right) \tilde{N}^{1},
\end{aligned}
$$

donde

$$
|x(t)| \leq-\frac{K}{\int_{0}^{t}|x(s)|{ }^{\prime} d s} \leq \frac{K}{\int_{s}^{t}|x(s)|{ }^{1} d s}=\frac{K}{\varphi(t)}
$$


daí,

$$
\begin{aligned}
|x(t)| & \leq \frac{K}{\varphi(s+1) e^{-1 / K} e^{(t-s) / K}}=\leq \frac{K e^{1 / K} e^{-(1-s) / K}}{|x(s)|^{-1} \tilde{M}^{-1}\left(1-e^{-\bar{N}}\right) \tilde{N}^{-1}}= \\
& =\frac{K \tilde{M} \tilde{N} e^{1 / K}}{1-e^{-\tilde{N}}} e^{(u s) / K}|x(s)| .
\end{aligned}
$$

Para $0 \leq t-s \leq 1$, temos $e^{1 / K} e^{-(t-s) / K} \geq 1$. Assim,

$$
x(t)\left|\leq e^{1 / K} e^{-(t-s) / K}\right| x(t) \mid
$$

e, utilizando (5.16), obtemos

$$
|x(t)| \leq \tilde{M} e^{1 / K+\tilde{N}} e^{\cdot(t-s) / K}|x(s)|
$$

Daí, combinando (5.18) e (5.19), obtemos

$$
|x(t)| \leq K_{1} e^{-\alpha_{1}(t \cdot s)}|x(s)|, \quad 0 \leq s \leq t<\infty,
$$

onde $K_{l}=\max \left\{\frac{\tilde{M} \tilde{N} K e^{1 / K}}{1-e^{-\tilde{N}}}, \tilde{M} e^{1 / K-\tilde{N}}\right\}$ e $\alpha_{1} \cdots \frac{1}{K}$.

Analogamente, consideramos o caso onde a solução da EDI (3.1), (3.2) se inicia $\mathrm{cm} x(0) \epsilon$ Ker $P$. Neste caso, para cada $a \in \mathbb{R}$, no lugar de $y_{a}(t)$, consideramos a função

$$
\tilde{y}_{a}(t)=\left[\left.\int_{t}^{\infty} \chi_{a}(\tau) \cdot x(\tau)\right|^{-1} d \tau\right] x(t)
$$

c obtemos

$$
|x(t)| \leq K_{2} e^{-\alpha_{2}(t-s)}|x(s)|, \quad 0 \leq s \geq t<\infty .
$$

Finalmente, podemos notar que (5.20) e (5.21) implicam em (5.14) e (5.15), onde $K=\max \left\{K_{1} . K_{2}\right\}$ e $\alpha=\min \left\{\alpha_{1}, \alpha_{2}\right\}$ (veja a Seção 3.1). Isto encerra a demonstração.

Observação 5.3 A condição $g(X)<\infty$ será satisfeita, se

$$
\sup _{t>0} \int_{t}^{t+1}\|A(s)\| d s<\infty \quad e \quad \sup _{0<t<\infty} \sum_{t \leq t_{n} \leq t+1}\left\|Q_{n}\right\|<\infty .
$$


A condição $g^{\prime}(X)>-\infty$ será satisfeita, se

$$
\sup _{t>0} \int_{t}^{t+1}\|A(s)\| d s<\infty \quad e \quad \sup _{0 \leq t<\infty_{t} \sum_{n} \leq 1+1} \mid Q_{n} \|^{-1}<\infty .
$$

Observação 5.4 As condiçōes

$$
g(X)<\infty \quad g^{\prime}(X)>-\infty
$$

seguem imediatamente da condição (i) do Teorema 5.2 juntamente com as seguintes condições

$$
\left.\sup _{t \geq 0}\right|_{i} A(t)\left\|<\infty, \quad \sup _{n \in \mathbb{N}}\right\| Q_{n}\left|<\infty . \quad \sup _{n \in \mathbb{N}}\right| Q_{n} \|\left.\right|^{-1}<\infty .
$$

Observação 5.5 O Teorema 5.4 continua válido se retirarmos a condição (ii) e impusermos a seguinte condição:

(ii') Para toda função $f \in \mathscr{L}_{p}\left(\mathbb{R}_{+}, X\right), 1 \leq p \leq \infty$, onde $\mathscr{L}_{p}\left(\mathbb{R}_{+}, X\right)$ denota o espaço de todas as funções $f: \mathbb{R}_{+} \rightarrow X$ satisfazendo

$$
\int_{\mathbb{R}_{+}}|f(t)|^{p} d t<\infty
$$

com a norma

$$
\|\left. f^{\prime}\right|_{p}=\left[\int_{\mathbb{E}_{+}}|f(t)|^{p} d t\right]^{1 / p}
$$

a EDI näo-homogênea (5.1), (5.2), com $h=0$, tem pelo menos uma solução iniciando no subespaço $\mathscr{L}_{p}\left(\mathbb{R}_{+}, X\right)$.

A prova pode ser obtida pelo mesmo caminho do Teorema 5.4.

\subsubsection{Admissibilidade de Funções com Limite no Infinito}

() objetivo desta subseção é caracterizar a dicotomia exponencial através da admissibilidade de um par de espaços de funções com limite no infinito. O resultado que apresentamos a seguir estabelece condições para que tenhamos a equivalência entre admissibilidade para certos pares de 
funções com respeito à EDI não-homogênea (5.1), (5.2) e a existência de uma dicotomia exponencial para sua equação homogênea correspondente, a EDI (3.1), (3.2). Uma parte do resultado é devida a Bainov et al (veja [1]). A nossa contribuição está em conseguirmos refinar o par de funções admissíveis.

\section{Teorema 5.5 19/ Suponhamos que.}

(i) A funçäo A seja limitada sobre $\mathbb{R}_{+}$;

(ii) A matriz A(t) seja invertível, para cada $t \in \mathbb{R}_{+}$;

(iii) det $Q_{n} \neq 0$, para qualquer $n \in \mathbb{N}$;

(iv) $\sup _{0 \leq l<\infty} \sum_{l \leq l_{n}<t-1}\left\|Q_{n, i}<\infty e \sup _{0<t<\infty} \sum_{\ell \leq t_{n} \leq l+1}\right\| Q_{n} \|^{-1}<\infty$.

Então as seguintes afirmações são equivalentes:

1. Opar $\left(\mathscr{B}^{0}\left(\mathbb{R}_{,}, X\right) . \mathscr{B}^{0}\left(\mathbb{R}_{+}, X\right)\right)$ é admissivel, isto é, dada $f \in \mathscr{P}^{0}\left(\mathbb{R}_{-}, X\right)$, a $E D I(5.1),(5.2)$ admite soluçăo $x \in \mathscr{P}^{0}\left(\mathbb{R}_{+}, X\right)$.

2. O par $\left(B^{0}(\infty), B_{A}^{0}(\infty)\right)$ é admissivel, isto é, dada $f \in \not^{0}(\infty)$, a $\operatorname{EDI}(5.1)$, (5.2) admite solução $x \in B_{A}^{0}(\infty)$.

3. A EDI (3.1), (3.2) tem uma dicotomia exponencial.

\section{Demonstração:}

(1. $\Leftrightarrow$ 3.) Segue diretamente dos Teoremas 5.3 e 5.4 , juntamente com a Obscrvação 5.3 . Notemos que csta cquivalência continua válida sem que as matrizes $A(t)$ sejam invertíveis.

$\left(1 . \Rightarrow\right.$ 2.) Seja $f \in \mathscr{A}_{+}(\infty)$. Como o par $\left(\mathscr{B}^{0}\left(\mathbb{R}_{+}, X\right), \mathscr{A}^{0}\left(\mathbb{R}_{+}, X\right)\right)$ é admissível, seguc, pela primeira parte desta demonstração, que a $\operatorname{LDI}(3.1),(3.2)$ tem uma dicotomia exponencial. Então, para qualquer $f \in \mathscr{B}\left(\mathbb{R}_{+} . X\right)$, a EDI $(5.1),(5.2)$ tem a seguinte solução limitada

$$
x(t)=\int_{0}^{t} U(t) P U^{-1}(s) f(s) d s-\int_{t}^{\infty} U(t)(I-P) U^{-1}(s) f(s) d s
$$


Utilizando a função de Green (veja (5.6)), podemos escrever a solução $x_{j}$ acima na forma

$$
x_{/}(t)=\int_{J} G(l, s) f(s) d s
$$

Devemos provar que $\lim _{t \rightarrow+\infty} f(t)$ existe. Como $A(t)$ é uma função limitada, podemos usar a seguinte identidade

$$
U(t) P U(0)-I=\int_{J} G(t, s) A(s) d s,
$$

que pode ser verificada sem maiores dificuldades.

Usando (5.22), podemos escrever

$$
x_{f}(t)=-A^{-1}(t) f(t)+U(t) P U^{-1}(0) A^{-1}(t) f(t)+I_{1}(t)+I_{2}(t)
$$

onde

$$
\left.I_{1}(t):=\int_{0}^{t} G(t, s) A(s)_{[}^{[} A^{\cdots !}(s) f(s)-A^{-1}(t) f(t)\right] d s
$$

c

$$
\left.I_{2}(t):=\int_{t}^{\infty} G(t, s) A(s)_{i}^{[} A^{-1}(s) f(s)-A^{-1}(t) f(t)\right] d s,
$$

De acordo com (3.8) e (3.9), podemos estimar $I_{1} \mathrm{e} I_{2}$. Temos

$$
\begin{aligned}
& I_{1}(t)_{i}=\left|\int_{0}^{t} G(t, s) A(s)\left[A^{-1}(s) f(s)-A^{-1}(t) f(t)\right] d s\right| \leq \\
& \leq\left.\int_{0}^{t}|| U(t) P U^{\cdots 1}(s)\right|_{i}|A(s)| i \mid A^{-1}(s) f(s)-A^{-1}(t) f(t) d s \leq \\
& \leq \int_{0}^{t} K e^{\alpha(t \cdot s)}\left|A \|_{\infty} \sup _{s \in\left[0, t_{j}\right.} A^{1}(s) f(s)-A^{-1}(t) f(t)\right| d s \leq \\
& \leq K|A|_{\infty}^{\prime} \alpha^{-1} \sup _{s \in[0, i j} \mid A^{-1}(s) f(s)-A^{1}(t) f(t) !\left[1+e^{-\alpha t}\right]
\end{aligned}
$$

$c^{2}$

$$
\begin{aligned}
& \left|I_{2}(t)\right|=\int_{1}^{\mid} G(t, s) A(s)\left[A^{-1}(s) f(s)-A^{-1}(t) f(t)\right] d s \mid \leq \\
& \leq \int_{t}^{\infty} ! U(t)(I-P) U^{-1}(s)|\cdot| A(s) \mid A^{-1}(s) f(s)-A^{-1}(t) f(t)^{\prime} d s \leq \\
& \leq\left.\int_{t}^{\infty} K e^{\alpha(\alpha-1)} ! \Lambda\right|_{, \infty} ^{1} \sup _{s=\{, \infty)} \mid \Lambda^{-1}(s) f(s)-A^{\prime \prime}(t) f(t)_{i} d s
\end{aligned}
$$




$$
\left|I_{2}(t)_{i} \quad \leq K \alpha^{-1}:\right| A_{!}^{\prime} \|_{\infty} \sum_{s \in t, \infty)} \mid A^{-1}(s) f(s)-A^{-1}(t) f(t)
$$

Como $f \in \mathscr{h}(\infty)$, por hipótese, segue, de (5.23) e (5.24), que $\lim _{i \rightarrow+\infty} I_{i}(t)-0, i=1,2$. De (5.22), temos que $\lim _{t \rightarrow \infty} x_{f}(t)--\left(A^{-1} f\right)(\infty)$. Portanto o par $\left(\mathscr{B}(\infty), \mathscr{R}_{A}(\infty)\right)$ é admissível.

(2. $\Rightarrow$ 1.) Notemos que, se $x_{1}(t)$ e $x_{2}(t)$ forem duas soluções da EDI não-homogênea (5.1), (5.2), então sua diferença, $z(t)=x_{1}(t)-x_{2}(t)$, será uma solução da LDI homogênea (3.1), (3.2) Se as soluções $x_{1}(t), x_{2}(t) \in \mathscr{B} A(\infty)$, cntão $z(t) \in \mathscr{B}(\infty)$ e, portanto, $z(0) \in X_{1}$. Se $x(t)$ for uma solução de (5.1), (5.2) em $h(\infty)$, então $\tilde{x}(t)=x(t)-U(t) P x(0)$ também será uma solução de (5.1), $(5.2) \mathrm{em} \mathscr{B}(\infty)$, com valor inicial $\tilde{x}(0)=(I \cdots P) x(0) \in X_{2}$.

Por hipótese, para $f \in \mathscr{B}_{\mathrm{A}}(\infty)$, a EDI não-homogênea (5.1), (5.2) tem uma única solução $\tilde{x}(t) \in$ $\mathscr{A}(\infty)$ satisfazendo a igualdade $P \tilde{x}(0)=0$. Assim, definimos o operador linear $\tilde{K}: \mathscr{B} A(\infty) \rightarrow \mathscr{B}(\infty)$ pela associação de cada elemento $f \in \mathscr{B}_{A}(\infty)$ a uma solução da EDI não-homogênea (5.1), (5.2).

Agora, provemos que $\tilde{K}$ tem gráfico fechado. Suponhamos que $f_{m} \rightarrow f$ em $\mathscr{B}_{A}(\infty)$ e que $y_{m}=\tilde{K} f_{m} \rightarrow y$. Então

$$
y(0)=\lim _{m \rightarrow \infty} y_{n}(0) \in X_{2}
$$

e, para qualquer $t$ fixado, temos

$$
\int_{0}^{t} f(s) d s=\lim _{m \rightarrow \infty} \int_{0}^{t} f_{m}(s) d s .
$$


Portanto,

$$
\begin{aligned}
y(t)-y(0) & =\int_{0}^{t} y^{\prime}(s) d s= \\
& =\int_{0}^{t_{1}} y^{\prime}(s) d s+\sum_{i=1}^{n-1} \int_{t_{i}}^{t_{i+1}} y^{\prime}(s) d s+\int_{t_{n}}^{t} y^{\prime}(s) d s= \\
& =\int_{0}^{t_{1}} \lim _{m \rightarrow \infty} y_{m}^{\prime}(s) d s+\sum_{i=1}^{n} \int_{t_{i}}^{t_{t \rightarrow 1}} \lim _{m \rightarrow \infty} y_{m}^{\prime}(s) d s+\int_{t_{n}}^{t} \lim _{m \rightarrow \infty} y_{m}^{\prime}(s) d s= \\
& =\lim _{m \rightarrow \infty} \int_{0}^{t_{1}} y_{m}^{\prime}(s) d s+\sum_{i=1}^{n} \lim _{m \rightarrow \infty} \int_{t_{i}}^{t_{i+1}} y_{m}^{\prime}(s) d s+\lim _{m \rightarrow \infty} \int_{t_{n}}^{t} y_{m}^{\prime}(s) d s= \\
& \left.\left.=\lim _{m \rightarrow \infty} \int_{0}^{t_{1}}\left[A(s) y_{m}(s)+f_{m}(s)\right]\right) d s+\sum_{i=1}^{n-1} \lim _{m \rightarrow \infty} \int_{t_{i}}^{t_{i+1}}\left[A(s) y_{m}(s)+f_{m}(s)\right]\right) d s+ \\
& +\lim _{m \rightarrow \infty} \int_{t_{n}}^{t}\left[A(s) y_{m}(s)-f_{m}(s)^{\prime} d s\right.
\end{aligned}
$$

daí

$$
\begin{aligned}
y(t)-y(0) & \left.-\int_{0}^{t_{1}}[A(s) y(s)+f(s)]\right) d s+\sum_{i=1}^{n-1} \int_{t_{i}}^{t_{i+1}}[A(s) y(s)+f(s)] d s+ \\
& +\int_{t_{n}}^{t}\left[A(s) y(s)+f(s)^{t}\right] d s= \\
& =\int_{0}^{l}[A(s) y(s)+f(s)] d s,
\end{aligned}
$$

E, como

$$
y\left(t_{n}^{+}\right)=\lim _{m \rightarrow \infty} y_{m}\left(t_{n}^{-}\right)=\lim _{m \rightarrow \infty} Q_{n} y_{m}\left(t_{n}\right)=Q_{n} y\left(t_{n}\right),
$$

segue que $y(t)$ é uma solução da equação (3.1), (3.2). Portanto, pelo Teorema do Gráfico Fechado de Banach, este operador é limitado, isto é, existe uma constante positiva $M$ tal que

$$
|\tilde{K}(f)|_{\infty}^{\infty}<M\|f\|_{A} .
$$

Seja $f \in \mathscr{B}(\mathbb{R}:, X)$ e, para cada $m-1,2 \ldots$, seja $\theta_{m}(t)$ uma função contínua tal cue $\left\|\theta_{m}\right\|_{\infty}=1$

$$
\theta_{m}(t)= \begin{cases}1, & t \in[0, m], \\ 0, & t \geq m+1 .\end{cases}
$$

Seja, também, $\left\{f_{m}\right\}$ uma seqüência em $\mathscr{B}_{A}(\infty)$ definida por

$$
f_{m}(t)=\theta_{m}(t) f(t)
$$


Para cada função $f_{m}$, consideremos a solução $x^{n}=\tilde{K}\left(f_{m}, 0\right)$ da EDI

$$
\begin{gathered}
\frac{d x}{d t}=A(t) x(t)+f_{n}(t), \quad t \neq t_{n}, \\
x\left(t_{n}^{+}\right)=x\left(t_{n}\right)+I_{n}\left(x\left(t_{n}\right)\right)=Q_{n} x\left(t_{n}\right) .
\end{gathered}
$$

De acordo com (5.25), para qualquer índice $m$, temos

$$
\left\|x^{m}\right\|_{\infty} \leq M\left\|_{\mid} f_{m}\right\|_{A} \leq M\|f\|_{A}
$$

Por (5.27), (5.28) e (5.29), obtemos seqüências $\left\{x^{m}\right\}_{m \in \mathbb{N}}$ e $\left\{\left(x^{m}\right)^{\prime}\right\}_{m \in \mathbb{N}}$ em $\mathscr{B}\left(\mathbb{R}_{+}, X\right)$, que são limitadas sobre qualquer subintervalo compacto de $\mathbb{R}_{+}$. Pelo Teorema de Áscoli-Arzelá para funções contínuas por partes (veja Apêndice 1) aplicado a cada intervalo compacto de $\mathbb{R}_{+}$, segue que existe

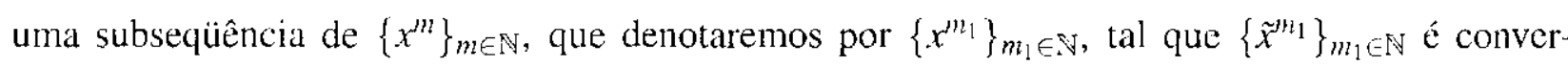
gente para uma funçăo $v_{!} \in \mathscr{C}\left(\left[0, t_{1}\right], X\right)$. Pelo mesmo argumento, existe uma subsequiência de $\left\{x^{m_{1}}\right\}_{m_{1} \subset \mathbb{N}}$, quc denotaremos por $\left\{x^{m_{2}}\right\}_{m_{2} \in \mathbb{N}}$, tal que $\left\{\tilde{x}^{m_{2}}\right\}_{m_{2} \in \mathbb{N}}$ é convergente para uma função $v_{2} \in \mathscr{C}\left(\left[t_{1}, t_{2}\right], X\right)$. Continuando com este procedimento, concluímos que, para cada número natural $n$. existe uma subseqüência de $\left\{x^{m_{n-1}}\right\}_{m_{n-1} \in \mathbb{N}}$, que denotaremos por $\left\{x^{m_{n}}\right\}_{m_{t i} \subset \mathbb{N}}$, tal que $\left\{\tilde{x}^{m_{n}}\right\}_{m_{n} \in \mathbb{N}}$ é convergente para uma funçāo $v_{n} \subset \mathscr{C}\left(\left[t_{n-1}, t_{n}\right], X\right)$. F́ claro que cada subsequiência $\left\{x^{m_{n}}\right\}_{m_{n} \in \mathbb{N}}$ convergente para uma função $u \in \mathscr{B}\left(\mathbb{R}_{+}, X\right)$, onde $u$ é uma função tal que $\tilde{u}_{i}=v_{i}$, para cada $i=1,2, \ldots$ De (5.26), (5.27) e (5.28), segue que u satisfa $\%$

$$
\begin{gathered}
u^{\prime}(t)=A(t) u(t)+f(t), \quad t \neq t_{n} \\
u\left(t_{n}^{+}\right)=Q_{n} u\left(t_{n}\right) .
\end{gathered}
$$

Isto implica que u é uma solução de (5.1), (5.2) no espaço $\mathscr{B}^{0}\left(\mathbb{R}_{+}, X\right)$. 


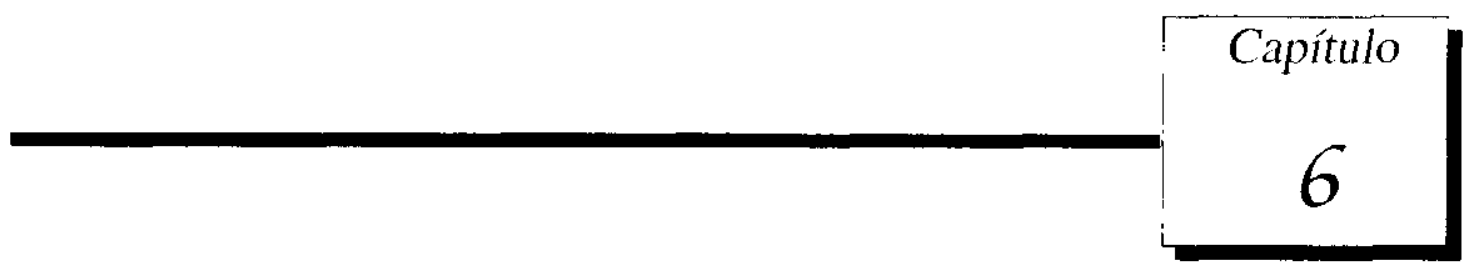

\section{Dicotomia Exponencial e Funções Quase Periódicas}

Neste capítulo, primeiramente apresentamos um resultado de D. D. Bainov et al, [1], que diz que se a $\operatorname{EDI}\left(3.1\right.$ ), (3.2) for quase periódica e tiver uma dicotomia exponencial sobre $\mathbb{R}_{++}$, então cla terá uma dicotomia exponencial sobre $\mathbb{R}$. O objetivo deste capítulo é mostrar um resultado inédito e mais geral que nos diz que se a EDI (3.1). (3.2) tiver uma dicotomia exponencial sobre um intervalo finito de comprimento suficientemente grande, então ela terá uma dicotomia sobre toda a reta.

Definição 6.1 Seja Y um espaço de Banach. Dizemos que $\gamma: \mathbb{R} \rightarrow Y$ é uma função quase periódica se, de qualquer sequiência $\left\{h_{v}\right\} \subset \mathbb{R}$, pudermos extrair uma subsequiência $\left\{h_{v_{k}}\right\}$ tal que o limite

$$
\lim _{k \rightarrow \infty} \gamma\left(t+h_{v_{k}}\right)
$$

exista uniformemente com respeito a $\in \mathbb{R}$.

Observação 6.1 A função $\gamma$ é quase periódica se, e somente se, para todo $\varepsilon>0$, existir $l(\varepsilon)>0$ tal que, para todo intervalo de comprimento $l(\varepsilon)$, pudermos encontrar um mímero $\omega$, chamado número E-translação para $A(t)$. satisfazendo

$$
|\gamma(t+\omega)-\gamma(t)|<\varepsilon,
$$


parat $\in \mathbb{R}$

O lema a seguir relaciona o operador de evolução da EDI (3.1), (3.2) com o operador de evolução da EDI (6.1), (6.2) abaixo, que nada mais é do que a EDI (3.1), (3.2) com uma adição em seus tempos.

Lema 6.1 //1], Lema 9.1/ Consideremos a EDI (3.1), (3.2) sobre $\mathbb{R}$ com $\operatorname{det} Q_{n} \neq 0$, para qualquer $n \in \mathbb{Z}$. Suponhamos que h seja uma constante positiva dada. Então a seguinte EDI

$$
\begin{aligned}
& \frac{d x}{d t}=A(t+h) x, \quad t \neq t_{n}, \\
& x\left(t_{n}^{+}\right)=Q\left(t_{n}+h\right) x\left(t_{n}\right) .
\end{aligned}
$$

terí um operador de evolução, $V_{h}(t)$, satisfazendo

$$
V_{h}(t)=U(t+h) U^{\cdot 1}(h)
$$

onde U(t) é a matriz findamental da EDI (3.1), (3.2).

Demonstração: Basta observarmos que

$$
\begin{aligned}
\frac{d V_{h}(t)}{d t}\left[V_{h}(t)_{j}^{-1}\right. & -\frac{d U(t+h) U^{-1}(h)}{d t}\left[U(t+h) U^{-1}(h)\right]^{\prime}- \\
& =\frac{d U(t+h)}{d t} U^{1}(h) U(h) U^{-1}(t+h)= \\
& =\frac{d U(t+h)}{d t} U^{-1}(t+h)
\end{aligned}
$$

donde segue o resultado.

O próximo lema relaciona os operadores de Cauchy das EDIs (3.1), (3.2) e (3.25), (3.26), através das estimativas (6.3), (6.4) e (6.5) seguintes.

Lema 6.2 (Il], Lema 9.2] Consideremos as EDIs (3.1), (3.2) e (3.25), (3.26). Sejam U(t.s) o 
operador de Cachy da EDI (3.1), (3.2) eV(t,s) o operador de Cachy da EDI (3.25), (3.26). Se

$$
\| U(t, s) ! \mid \leq N e^{-v(h(t)-h(s))} .
$$

para quaisquer $t, s \in \mathbb{R}$, onde $h(t), t \subset \mathbb{R}$, é uma funçäo qualquer satisfazendo $h(0)=0$, então teremos

$$
\|V(t)\| \leq N e^{-V h(t)} e^{N \int_{0}^{t} p(\tau) d \tau} \prod_{0<i j<t}\left(1+q\left(t_{j}\right)\right)
$$

e

$$
\begin{aligned}
\|V(t)-U(t)\| & \leq N e^{-v h(t)} \Delta(t)\left(e^{N \int_{0}^{\prime} p(v) d s}-1\right)+ \\
& +N e^{v h(t)} \sum_{0<t_{j}<t} N q\left(t_{j}\right) e^{N \int_{0}^{t_{j}} p(\tau) d \tau} \prod_{0<t_{k}<t_{j}}\left(1+q\left(t_{k}\right)\right),
\end{aligned}
$$

onde

$$
\begin{gathered}
q(t)=\sum_{n}\left\|Q_{n}-R_{n}\right\| \delta\left(t-t_{n}\right) \\
\Delta(t)=\prod_{0<t_{t}<t}\left(1+q\left(t_{i}\right)\right), \\
p(t)=\|B(t)-A(t)\|
\end{gathered}
$$

e $\delta(t)$ é a função característica do conjunto $\{0\}$.

Demonstração: Notemos que o operador $V(t)=V(t, 0)$ é uma solução da EDI

$$
\begin{gathered}
\frac{d W}{d t}-A V=(B-A) V . \quad t \neq t_{n}, \\
W\left(t_{n}^{+}\right)=Q_{n} W\left(t_{n}\right)+\left(R_{n}-Q_{n}\right) W\left(t_{n}\right) \\
W(0)=I .
\end{gathered}
$$

Pela Fórmula da Variação das Constantes (Lema 2.1), temos

$$
\begin{aligned}
X(t)= & U(t, 0)+\int_{0}^{t} U(t, \tau)(B(\tau)-A(\tau)) V(\tau) d \tau+ \\
& \sum_{0<t_{j}<t} U\left(t, t_{j}^{+}\right)\left(R_{j}-Q_{j}\right) V\left(t_{j}\right),
\end{aligned}
$$


onde $X(t)$ é uma solução do problema de Cauchy $(6.6),(6.7),(6.8)$.

Como o operador $V(t)$ é uma solução do problema de Cauchy (6.6), (6.7), (6.8), por (6.9), concluímos que

$$
\begin{aligned}
\|V(t)\| & \leq\|U(t, 0)\|+\int_{0}^{t}\left\|U(t, \tau)_{i}\right\|\|B(\tau)-A(\tau)\|\|V(\tau)\| d \tau+ \\
& +\sum_{0<t_{j}<t}\left\|U\left(t, t_{j}\right)\right\|\left\|Q_{j}-R_{j}\right\|\left\|V\left(t_{j}\right)\right\|
\end{aligned}
$$

Seja $\varphi(t)={ }_{1} V(t) \|$. Por (6.3), segue que

$$
\begin{aligned}
\varphi(t) & \leq N e^{-v h(t)}+N \int_{0}^{t} e^{-v(h(t)-h(\tau))} p(\tau) \varphi(\tau) d \tau+ \\
& +\sum_{0<t_{j}<t} e^{--v\left(h(t)-h\left(t_{j}\right)\right)} q\left(t_{j}\right) \varphi\left(t_{j}\right)
\end{aligned}
$$

e, fazendo $\varphi_{1}(t)=\varphi(t) e^{v / h_{(}(t)}$, chegamos a

$$
\varphi_{1}(t) \leq N+N \int_{0}^{t} \varphi_{1}(\tau) p(\tau) d \tau+\sum_{0<l_{j}<t} q\left(t_{j}\right) \varphi_{1}\left(t_{j}\right)
$$

Daí, aplicando o L.ema 2.3, temos

$$
\varphi_{1}(t) \leq N e^{N \int_{0}^{t} p(\tau) d \tau} \prod_{0<1<t}\left(1+q\left(t_{j}\right)\right)
$$

c, cntão,

$$
\varphi(t) \leq N e^{-v h(t)} e^{N \int_{0}^{t} p(\tau) d \tau} \prod_{0<t_{j}<t}\left(1+q\left(t_{j}\right)\right)
$$

Portanto a desigualdade (6.4) está provada.

Agora provaremos (6.5). Pelo fato do operador $V(t)$ ser uma solução do problema de Cauchy $(6.6),(6.7),(6.8)$, por $(6.9)$, temos

$$
\begin{aligned}
\|V(t) U(t)\| & =|| U(t, 0)+\int_{0}^{t} U(t, \tau)(B(\tau)-A(\tau)) V(\tau) d \tau+ \\
& \perp \sum_{0<t_{j}<t} U\left(t, t_{j}^{+}\right)\left(R_{j}-Q_{j}\right) V\left(t_{j}\right)-U(t, 0) \mid
\end{aligned}
$$


Daí,

$$
\begin{aligned}
& \|V(t)-U(t)\|_{i} \leq \int_{0}^{t}\|U(t, \tau)\|\|B(\tau)-A(\tau)\|\left\|\left.V(\tau)\right|_{i} d \tau+\sum_{0<t_{j}<t}\right\| U\left(t, t_{j}^{-}\right):\left\|Q_{j}-R_{j}\right\|\left\|V\left(t_{j}\right)\right\| \leq \\
& \leq \int_{0}^{t} \mid U(t, \tau)\left\|_{p} p(\tau) \varphi(\tau) d \tau+\sum_{0<t_{j}<t}\right\| U\left(t, t_{j}^{*}\right) \|_{1}^{i} q\left(t_{j}\right) \varphi\left(t_{i}\right) \leq \\
& \leq \int_{0}^{t} N e^{-v(h(t) h(\tau))} p(\tau) N e^{-v h(\tau)} e^{N \int_{0}^{\tau} p(s) d s} \prod_{0<1 j<\tau}\left(1+q\left(t_{j}\right)\right) d \tau+
\end{aligned}
$$

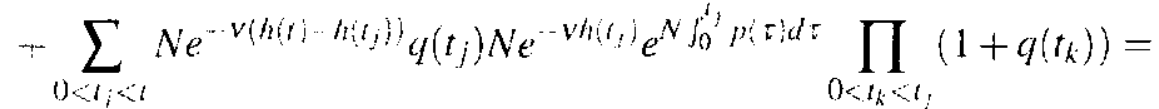

$$
\begin{aligned}
& =N e^{-v / h^{\prime}(t)} \int_{0}^{t} N p(\tau) e^{N \int_{0}^{\tau} p(s) d s} \prod_{0<t_{j}<\tau}\left(1+q\left(t_{j}\right)\right) d \tau+ \\
& +N e^{v /(t)} \sum_{0<t_{j}<i} N q\left(t_{j}\right) e^{N f_{0}^{t^{j}} p(\tau) d \tau} \prod_{0<t_{k}<t_{j}}\left(1+q\left(t_{k}\right)\right) \text {. }
\end{aligned}
$$

Como $\int_{a}^{b} N p(\tau) e^{N \int_{0}^{\tau} p(s) d s} d \tau-e^{\left.N \int_{0}^{b} p i s\right) d s}-e^{N \int_{0}^{u} p(s) d s}$, temos

$$
\begin{aligned}
& N e^{-v h(t)} \int_{0}^{t} N p(\tau) e^{N \int_{0}^{\tau} p(s) d s} \prod_{0<t_{j}<\tau}\left(1+q\left(t_{j}\right)\right) d \tau= \\
& =N e^{-V h(t)} \sum_{j=1}^{n i t)}\left\{\int_{t_{j-1}}^{t_{j}} N p(\tau) e^{N \int_{0}^{\tau} p(s) d s} \prod_{0<t_{j}<\tau}\left(1+q\left(t_{j}\right)\right) d \tau\right\}+ \\
& -N e^{\cdots v h(t)} \int_{t_{t i l} t}^{t} N p(\tau) e^{N \int_{0}^{\tau} p(t) d s} \prod_{0<t_{j}<\tau}\left(1+q\left(t_{j}\right)\right) d \tau \leq
\end{aligned}
$$

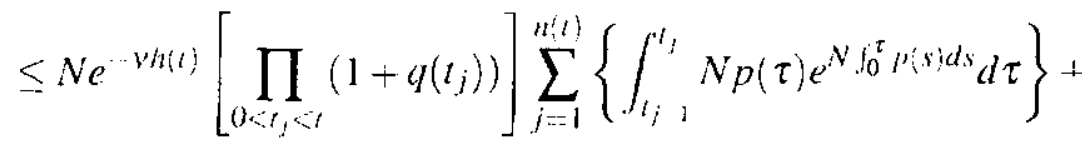

$$
\begin{aligned}
& +N e^{v h(t)}\left[\prod_{0<t_{j}<t}\left(1+q\left(t_{j}\right)\right)\right] \int_{t_{t, t} t}^{t} N p(\tau) e^{N \int_{0}^{\tau} p(s) d s} d \tau= \\
& =N e^{-v h(t)}\left[\prod_{0<t_{j}<t}\left(1+q\left(t_{j}\right)\right)\right] \sum_{j=1}^{n(t)}\left[e^{N \int_{0}^{t} p(v) d s}-e^{N \int_{0}^{j_{j}-1} p(s) d s}\right]+
\end{aligned}
$$

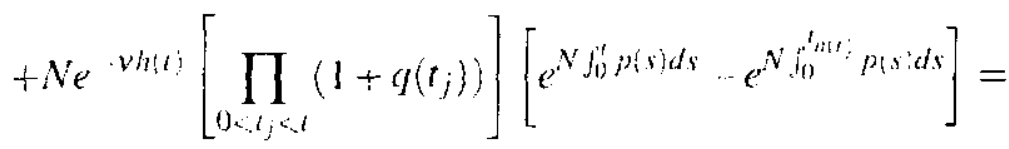

$$
\begin{aligned}
& =N e^{-v h(t)} \Delta(t)\left[e^{N \int_{0}^{l} p(s) d s}-e^{N l_{0}^{t_{0}} p(s) d s}\right]=N e^{-v h(t)} \Delta(t)\left[e^{N \int_{0}^{t} p(s) d s}-1\right] \text {, }
\end{aligned}
$$


donde segue o resultado.

Lema 6.3 //1], Lema 9.3/ Consideremos a EDI (3.1), (3.2) $\mathbb{R}$ e suponhamos que

(i) A EDI (3.1), (3.2) tenha uma dicotomia exponencial sobre a semi-reta $\mathbb{R}$. com constantes $K$ e $\alpha$ e projeçăo $P$

(ii) Exista uma seqüencia de números $\left\{h_{v}\right\}_{v \in \mathbb{N}}$ com $h_{v} \rightarrow \infty$ quando $v \rightarrow \infty$. tal que $\lim _{v \rightarrow \infty} A\left(t+h_{v}\right)=B(t)$ e $\lim _{v \rightarrow \infty} Q\left(t+h_{v}\right)=\tilde{Q}(t)$ ambos os limites uniformes sobre qualquer segmento finito da reta $\mathbb{R}$

(iii) $\operatorname{det} Q_{n} \neq 0$, para qualquer $n \in \mathbb{Z}$.

Então existirá uma projeção $\tilde{P}: X \rightarrow X$ tal que

$$
\lim _{v \rightarrow \infty} U\left(h_{V}\right) P U^{-1}\left(h_{v}\right)=\tilde{P}
$$

e a equação

$$
\begin{gathered}
\frac{d y}{d t}=B(t) y, \quad t \neq t_{n}, \\
y\left(t_{n}^{+}\right)=\tilde{Q}\left(t_{n}\right) y\left(t_{n}\right) .
\end{gathered}
$$

terá una dicotomia exponencial sobre a reta $\mathbb{R}$ com projeção $\tilde{P}$

Demonstração: Consideremos a equação

$$
\begin{gathered}
\frac{d x}{d t}=A\left(t+h_{v}\right) x, \quad t \neq t_{n}, \\
x\left(t_{n}^{-}\right)=Q\left(t-h_{v}\right) x\left(t_{n}\right) .
\end{gathered}
$$

com a matriz fundamental $V_{v}(t)$. De acordo com o I ema $6.1, V_{v}(t)$ satisfaz

$$
V_{v}(t)=U\left(t+h_{v}\right) U^{-1}\left(h_{v}\right)
$$


onde $U(s)$ denota a matriz fundamental de (3.1), (3.2).

Seja $P_{v}=U\left(h_{v}\right) P U^{-1}\left(h_{v}\right)$, onde $P$ é a projeção da dicotomia exponencial da EDI (3.1), (3.2). Então

$$
\left\|V_{v}(t) P_{v} V_{v}^{-1}(s)\right\|=\left\|U\left(t+h_{v}\right) U^{-1}\left(h_{v}\right) U\left(h_{v}\right) P U^{-1}\left(h_{v}\right) U\left(h_{v}\right) U^{-1}\left(s+h_{v}\right)\right\| .
$$

daí

$$
\left\|V_{v}(t) P_{v} V_{v}^{1}(s)|=| U\left(t-h_{v}\right) P U^{1}\left(s+h_{\nu}\right)\right\| \leq K e^{\alpha(t s)}
$$

para $-h_{v} \leq s \leq t<\infty$

Analogamente, prova-se que

$$
\| V_{v}(t)\left(I-P_{v}\right) V_{v}^{-1}(s) \mid \leq K e^{\alpha(s-t)}
$$

para $-h_{v} \leq s \leq t<\infty$

De (6.12) segue que, para todo $v \in \mathbb{Z},\left|P_{v}\right| \leq K$. Assim, podemos tomar uma subsequiência $\left\{P_{v_{n_{k}}}\right\}$ da seqüencia $\left\{P_{V}\right\}$ tal que exista

$$
\tilde{P}-\lim _{k \rightarrow \infty} P_{v_{k}}
$$

Podemos observar, pela própria definição, que $\tilde{P}$ é uma projeção.

Seja $W(t)$ a matriz fundamental da EDI $(6.10),(6.11)$. Pelo Lema 6.2 , segue que, para todo $l \in \mathbb{R}$.

$$
\lim _{v \rightarrow \infty} V_{v}(t)=W(t)
$$

Então por (6.12), fazendo $v \rightarrow \infty$, temos

$$
\left\|V_{v}(t) \tilde{P} V^{-1}(s)\right\| \leq K e^{-\alpha(t-s)} . \quad h_{v} \leq s \leq t<\infty
$$

e

$$
\| V_{v}(t)(I \quad \tilde{P}) V^{-1}(s) \mid<K e^{-\alpha(s-t)}, \quad-h_{v} \leq s \leq t<\infty,
$$


ou seja, a EDI (6.10), (6.11) tem uma dicotomia exponencial e a prova está concluída.

Considerando a $\mathrm{EDI}(3.1),(3.2)$ sobre $\mathbb{R}$, o próximo teorema diz que se $A(t)$ e $Q(t)$ forem quase periódicas sobre $\mathbb{R}$ e se a EDI (3.1), (3.2) admitir uma dicotomia exponencial sobre $\mathbb{R}$ + então ela terá uma dicotomia exponencial sobre $\mathbb{R}$.

Teorema 6.1 [l1], Teorema 9.1] Suponhamos que.

(i) AEDI (3.1), (3.2) sobre $\mathbb{R}$ admita uma dicotomia exponencial sobre a semi-reta $\mathbb{R}_{+}$.

(ii) As funções $A(t)$ e $Q(t)$ sejam quase periódicas sobre a reta.

(iii) $\operatorname{det} Q_{n} \neq 0$, para qualquer $n \in \mathbb{Z}$.

Então a EDI (3.1), (3.2) térá uma dicotomia exponencial sobre a reta $\mathbb{R}$.

Demonstração: Como $A(t)$ e $Q(t)$ são funções quase periódicas sobre a reta, segue que existe uma seqüência $h_{v} \rightarrow \infty$, quando $v \rightarrow \infty$, tal que $A\left(t+h_{v}\right) \rightarrow A(t)$ e $Q\left(t+h_{v}\right) \rightarrow Q(t)$, quando $v \rightarrow \infty$ uniformemente. Desta forma, o teorema segue diretamente do Lema 6.3.

O lema a seguir nos dá estimativas para funções que satisfazem algumas propriedades. Estas estimativas serão importantes para mostrarmos que a EDI (3.1), (3.2) admite uma dicotomia exponencial.

Lema 6.4 (i) Suponhamos que $x: \mathbb{R}_{+} \rightarrow X$ seja contínua por partes, com descontinuidade de primeira espécie em $t_{n}$ e contínuas à esquerda em $t_{n}, n \in \mathbb{N}$, com $x(t) \neq 0$, para todo $t \in \mathbb{R}_{+}$. Se existirem constantes $C \geq 1, \theta>1$ e $h>0$ tais que

$$
|x(t)| \leq C|x(s)|, \quad 0<t \leq s+h
$$

$e$

$$
|x(t)| \geq \theta \inf \{|x(s)|:|t-s| \leq h\}, \quad t \geq h
$$


Então existirão constantes $\alpha>0$ e $K \geq 1$ tais que

$$
|x(t)| \leq K e^{-\alpha(t-s)}|x(s)|, \quad 0 \leq s \leq t,
$$

$o u$

$$
|x(t)| \leq K e^{-\alpha(s-r)}|x(s)|, \quad 0 \leq t \leq s
$$

(ii) Suponhamos que $x: \mathbb{R}_{-} \rightarrow X$ seja contínua por partes, com descontinuidade de primeira espécie em $t_{n}$ e contínuas à esquerda em $t_{n}, n \in \mathbb{Z}_{-}, \operatorname{com} x(t) \neq 0$, para todo $t \in \mathbb{Z}_{-}$. Se existirem constantes $C \geq 1, \theta>1$ e $h>0$ tais que

$$
|x(t)| \leq C|x(s)|: \quad s \leq t \leq s+h \leq 0
$$

$$
|x(t)| \geq \theta \inf \{|x(s) !:| t-s \mid \leq h\}, \quad t \leq-h
$$

Então existirão constantes $\alpha>0$ e $K \geq 1$ tais que

$$
|x(t)| \leq K e^{-\alpha(t-s)}|x(s)|, \quad s \leq t \leq 0,
$$

ou

$$
|x(t)| \leq K e^{-\cdots \alpha(s-t)} \mid x(s) !, \quad t \leq s \leq 0
$$

Demonstração: Provaremos apenas a afirmativa (i) pois a prova da afirmativa (ii) é análoga.

Seja $\lambda: \mathbb{R}_{+} \rightarrow \mathbb{R}_{+}$definida por

$$
\lambda(s)=\inf \{x(u) \mid: u \geq s\}
$$

Dividiremos a prova em duas partes: Na primeira parte suporemos que $\lambda(0)>0$ e, na segunda parte, consideraremos que $\lambda(0)=0$. Em cada uma destas partes obteremos uma das estimativas que queremos.

Parte 1: Suponhamos, primeiramente, que $\lambda(0)>0$, isto é, que $\inf \{|x(u)|: u \geq 0\}>0$. 
Por hipótese, para $s \geq t \geq h$, temos

$$
\begin{aligned}
|x(t)| & \geq \theta \inf \{|x(s)|:|t-s| \leq h\}= \\
& =\theta \inf \{|x(s)|: s-h \leq t \leq s+h\} \geq \\
& \geq \theta \inf \{|x(s)|: t \geq s-h\}= \\
& =\theta \lambda(s-h) .
\end{aligned}
$$

Pela definição de $\lambda(s)$, existe uma seqüencia $\left\{u_{m}\right\}_{m \in \mathbb{N}}$, com $u_{m} \geq s$, tal que

$$
\lim _{m \rightarrow \infty} \mid x\left(u_{m}\right) !=\lambda(s)
$$

Provaremos, agora, que existe $u m m_{0} \in \mathbb{N}$ tal que $u_{m} \leq s+h$, para todo $m \geq m_{0}$. Suponhamos o contrário, isto é, que exista uma subseqüência $\left\{u_{m_{k}}\right\}_{m_{k} \in \mathbb{N}}$ de $\left\{u_{m}\right\}_{m \in \mathbb{N}}$ tal que $u_{m_{k}}>s+h$. Então

$$
\begin{aligned}
\left|x\left(u_{m_{k}}\right)\right| & \geq \theta \inf \left\{|x(u)|:{ }_{i} u-u_{m_{k}} \mid \leq h\right\}= \\
& =\theta \inf \left\{|x(u)|: u_{m_{k}}-h \leq u<u_{m_{k}}+h\right\}> \\
& \geq \theta \inf \left\{\mid x(u) !: u \geq u_{m_{k}} \cdots h\right\}= \\
& =\theta \lambda\left(u_{m_{k}}-h\right) \geq \theta \lambda(s)>\lambda(s) .
\end{aligned}
$$

Mas isto contradiz (6.13). Portanto existe um $m_{0} \in \mathbb{N}$ tal que $u_{m} \leq s+h$, para $m \geq m_{0}$. Então

$$
\lambda(s)=\inf \{|x(u)|: s \leq u \leq s+h\}
$$

e, para $0 \leq s-2 h<2+h$ e $s \leq t \leq s+h$, temos

$$
\begin{aligned}
|x(s)| & \geq \theta^{2} \inf \{|x(u)|:|u-s| \leq 2 h\} \geq \\
& \geq \theta^{2} \lambda(s \cdots 2 h) \geq \\
& \geq \theta^{2} \inf \{|x(u)|: s \cdots h \leq u \leq s+h\} \geq \\
& \geq \theta^{2} C^{-1}|x(t)| .
\end{aligned}
$$


Assim, para $t+(k-1) h \leq s<t+k h$, temos

$$
\begin{aligned}
|x(s)| & \geq \theta \inf \{|x(u):| u-s \mid \leq h\} \geq \\
& \geq \theta^{2} \inf \{|x(u)|:|u-s| \leq 2 h\} \geq \ldots \geq \\
& \geq \theta^{k+1} \inf \{|x(u)|:|u-s| \leq(k+1) h\} \geq \\
& \geq \theta^{k+1} \lambda(s-(k+1) h)= \\
& =\theta^{k+1} \inf \{|x(u)|:|s-(k+1) h| \leq u \leq s-k h\} \geq \\
& \geq \theta^{k+1} C^{-1}|x(t)| .
\end{aligned}
$$

Tomando $\alpha=h^{-1} \ln \theta$ e $K_{1}=\max \left\{1, C \theta^{-1}\right\}$, temos

$$
|x(t)| \leq K_{1} e^{\alpha(s-1)}|x(s)|, \quad 2 h \leq t \leq s
$$

Scja

$$
K=\beta_{1} e^{2 \alpha h h_{h} \max \{|x(t)|: t \in[0,2 h]\}}, .
$$

Então

$$
|x(t)| \leq K e^{-\alpha(s-t)}, x(s) !, \quad 0 \leq t \leq s
$$

Parte 2: Suponhamos, agora, que $\lambda(0) \div 0$, isto é, que inf $\{|x(u)|: u \geq 0\} \div 0$

Construamos uma sequência $\left\{\tau_{m}\right\}_{m \in \mathbb{N}}$ tal que

$$
\left|x\left(\tau_{m}\right)\right|=\theta^{-m} C|x(0)|
$$

$$
|x(t)|>\theta^{-m} C \mid x(0) !, \quad 0 \leq t<\tau_{m} .
$$

Assim, $h \leq \tau_{1}<\tau_{2}<\ldots<\tau_{m}<\tau_{m-1}<\ldots$

Agora, provaremos que $\tau_{m: 1}>\tau_{m}+h$. Suponhamos, por absurdo, que exista um $m_{0} \in \mathbb{N}$ tal que $\tau_{m_{(}+1} \leq \tau_{m_{0}}+h$. Então 


$$
\begin{aligned}
\left|x\left(\tau_{m_{0}+1}\right)\right| & <\inf \left\{|x(u)|: 0 \leq u \leq \tau_{m_{0}+1}+h \mid\right\}= \\
& \leq \inf \left\{x(u)::\left|u-\tau_{m_{0}+1}\right| \leq h\right\} \leq \\
& \leq \theta^{-1} \mid x\left(\tau_{m_{0}+1}\right) !
\end{aligned}
$$

o que contradiz o fato de que $\left|x\left(\tau_{m_{0}+1}\right)\right|=\theta^{-1} x\left(\tau_{m_{0}}\right) \mid$. Assim, $\tau_{m+1}>\tau_{m}+h$.

Seja $t \geq s \geq 0$. Logo existem inteiros positivos $k$ e $m$ tais que $\tau_{m} \leq t \leq \tau_{m-1}$ e $\tau_{k} \leq s \leq \tau_{k+\mathrm{I}}$. Então

$$
|x(s)|>x\left(\tau_{k+1}\right)\left|=\theta^{m-k-1}\right| x\left(\tau_{m}\right)\left|\geq \theta^{m-k-1} C^{-1}\right| x(t)\left|=\theta^{m-k+1} \theta^{-2} C^{-1}\right| x(t) \mid
$$

Como

$$
\tau_{m} \leq t \leq \tau_{m+1} \quad \text { e } \quad \tau_{k} \leq s \leq \tau_{k+1},
$$

temos

$$
t-s \leq \tau_{m+1}-\tau_{k} \leq(m-k+1) h
$$

e, assim,

$$
-(t-s) \geq-(m-k+1) h .
$$

Daí,

$$
|x(t)| \leq \theta^{2} C e^{-\left(h^{-1} \ln \theta\right)(t-s)}
$$

Tomando $\alpha=h^{1} \ln \theta$ e $\left.K_{\mathrm{l}}=C \theta^{2}\right\}$, segue que

$$
|x(t)| \leq K_{1} e^{-\alpha(t-s)} x(s) \mid, \quad \tau_{1} \leq s \leq t .
$$

Seja

$$
K=\beta_{1} e^{2 \alpha h} \frac{\max \{|x(t)|: t \in[0,2 h]\}}{\min \{x(t) \mid: t \in[0,2 h]\}}
$$

Então

$$
|x(t)| \leq K e^{-\alpha(t-s)}|x(s)|, \quad 0 \leq s \leq t
$$

Isto completa a prova do lema 
O lema a seguir apresenta propriedades de uma EDI que admite uma dicotomia exponencial.

Lema 6.5 Consideremos a EDI (3.1), (3.2) sobre $\mathbb{R}$. Suponhamos que o operador A seja limitado e que det $Q_{n} \neq 0$, para $n \in \mathbb{Z}$. Se a $E D I(3.1)$, (3.2) tiver uma dicotomia exponencial então, para todo $\theta>1$, existirão uma constante $h>0$ e $N$ soluçōes linearmente independentes da EDI (3.1), (3.2), digamos $x_{1}(t), x_{2}(t), \ldots, x_{N}(t)$, tais que

$$
\begin{gathered}
\left|x_{i}(t)\right| \geq \theta \inf \left\{\left|x_{i}(u)\right|:|u-t| \leq h\right\}, \quad i=1,2, \ldots, N, \\
\varlimsup_{i \rightarrow-\infty}\left|x_{i}(t)\right|>0, \quad i=1,2, \ldots, k
\end{gathered}
$$

$e$

$$
\varlimsup_{t \rightarrow \infty}\left|x_{i}(t)\right|>0, \quad i=k+1, k+2, \ldots, N
$$

Demonstração: Suponhamos que a $\operatorname{EDI}(3.1)$, (3.2) tenha uma dicotomia exponencial. Logo existem contantes $\alpha>0, K \geq 1$ e $N$ soluçōes linearmente independentes $x_{1}(t), x_{2}(t), \ldots, x_{N}(t)$, tais que

$$
\left|x_{i}(t)\right| \leq K e^{-\alpha(t-s)}\left|x_{i}(t)\right|, \quad t \geq s, i=1,2, \ldots, k
$$

e

$$
\left|x_{i}(t)\right| \leq K e^{-\alpha(x-t)}\left|x_{i}(t)\right|, \quad t \leq s, i=k+1, k+2, \ldots, N
$$

donde

$$
\varlimsup_{i \rightarrow-\infty}\left|x_{i}(t)\right|=+\infty, \quad i=1,2 \ldots, k
$$

e

$$
\varlimsup_{i \rightarrow 1 \infty} x_{i}(t) \mid=+\infty, \quad i=k+1 . k-2 \ldots, N
$$

Desta forma precisamos provar apenas que, dado $\theta>1$, existe $h>0$ tal que

$$
x_{i}(t) \mid \geq \theta \inf \left\{\left|x_{i}(u)\right|: u-t<h\right\}, \quad i=1.2, \ldots, N
$$

Seja $\theta>1$ fixo e tomemos $h>0$ tal que $K^{-1} e^{\alpha h}=\theta$. Assim, para $i=1.2 \ldots k$, temos

$$
\left|x_{i}(t)\right| \geq K^{1} e^{\alpha h}\left|x_{i}(t+h)\right|=\theta: x_{i}(t+h) \mid \geq \theta \inf \left\{\left|x_{i}(u)\right|:|u-t| \leq h\right\}
$$


E, para $i=k-1, k+2 \ldots, N$, temos

$$
\left|x_{i}(t)\right| \geq K^{-1} e^{\alpha h} x_{i}(t-h)|=\theta| x_{i}(t-h) \mid \geq \theta \inf \left\{\left|x_{i}(u)\right|:|u-t| \leq h\right\} .
$$

donde segue o resuldado.

O próximo lema é uma recíproca do lema anterior. É importante salientarmos que, no lema anterior, conseguimos o fato de que, sob certas hipóteses, se a EDI (3.1), (3.2) admitir uma dicotomia exponencial, então para toda constante $\theta>1$, conseguiremos encontrar uma constante $h>0$ c $N$ soluções linearmente independentes da EDI (3.1), (3.2), digamos $x_{1}(t), x_{2}(t), \ldots, x_{N}(t)$, de modo que algumas propriedades sejam válidas. Por outro lado, no lema a seguir, se existirem constantes $\theta>1, h>0$ e $N$ soluções linearmente independentes da EDI (3.1), (3.2), digamos $x_{1}(t), x_{2}(t), \ldots, x_{N}(t)$, que satisfaçam algumas propriedades, então a EDI (3.1), (3.2) admitirá uma dicotomia exponencial.

Lema 6.6 Consideremos a EDI (3.1), (3.2) sobre $\mathbb{R}$. Suponhamos que o operador A seja limitado e que $\operatorname{det} Q_{n} \neq 0$, para $n \in \mathbb{Z}$. Suponhamos, ainda, que existam constantes $\theta>1$ e $h>0$ e $N$ soluçōes linearmente independentes da EDI (3.1), (3.2), digamos $x_{1}(t), x_{2}(t), \ldots, x_{N}(t)$, tais que

$$
\begin{gathered}
\left|x_{i}(t)\right| \geq \theta \inf \left\{x_{i}(u)|:| u-t \mid \leq h\right\}, \quad i=1,2, \ldots, N, \\
\varlimsup_{t \rightarrow \infty}\left|x_{i}(t)\right|>0, \quad i=1,2, \ldots, k
\end{gathered}
$$

$e$

$$
\varlimsup_{i \rightarrow+\infty}\left|x_{i}(t)\right|>0, \quad i=k+1, k+2, \ldots, N
$$

Enãos a EDI (3.1), (3.2) terá tma dicotomia exponencial

Demonstração: Assumamos que exista uma constante $h>0$ tal que o operador $A$ da EDI (3.1), (3.2) seja limitado, daí

$$
\left|x_{i}(t)\right| \leq C \mid x_{i}(s) ! \quad s \leq t \leq s+h, \quad i=1,2, \ldots, N
$$

Para $i-1,2 \ldots k \mathrm{e} t \in \mathbb{R}_{+}$, como $\varlimsup_{t \rightarrow \cdots \infty}\left|x_{i}(t)\right|>0$, segue pelo Lema 6.4 que existem constantes $\alpha_{1}>0$ e $K_{1} \geq 1$ tais que 


$$
\left|x_{i}(t) \leq K_{1} e^{-\alpha_{1}(t-s)} \cdot x_{i}(s)\right|, \quad 0 \leq s \leq t
$$

Novamente, pelo Lema 6.4 , para $t \in \mathbb{R}_{-}$, temos que existem constantes $\alpha_{2}>0 \mathrm{e} \beta_{2} \geq 1$ tais que

$$
\left|x_{i}(t)\right| \leq K_{2} e^{--\alpha_{2}(t \cdots s)}\left|x_{i}(s)\right|, \quad s \leq t \leq 0
$$

ou

$$
\left|x_{i}(t)\right| \leq K_{2} e^{\alpha_{2}(s-r)}\left|x_{i}(s)\right|, \quad t \leq s \leq 0
$$

Queremos mostrar que apenas (6.15) é verdadeiro. Suponhamos, por absurdo, que (6.16) seja verdadeiro, disto e por (6.14) temos

$$
\varlimsup_{t \rightarrow-\infty}\left|x_{i}(t)\right|=0 \quad \text { e } \quad \varlimsup_{t \rightarrow-\infty} x_{i}(t) \mid=0 .
$$

Logo, existe um $t_{0} \in \mathbb{R}$ tal que

$$
x_{i}\left(t_{0}\right) \mid=\sup \left\{\left|x_{i}(u)\right|: u \in \mathbb{R}\right\} .
$$

Isto contradiz a condição

$$
\left|x_{i}(t)\right| \geq \inf \left\{\left|x_{i}(u)\right|:|u-t| \geq h\right\}
$$

Como uma das duas condiçôes necessariamente acontece, segue que apenas (6.15) é verdadeiro.

$$
\text { Sejam } \alpha=\min \left\{\alpha_{1}, \alpha_{2}\right\} \text { e } K=\max \left\{K_{1}, K_{2}\right\} \text {, por (6.14) e (6.15) obtemos }
$$

$$
\left|x_{i}(t): \leq K e^{\alpha(t} x^{s}\right| x_{i}(s) \mid, \quad s \leq t
$$

Analogamente, provamos que

$$
\left|x_{i}(t)\right| \leq K e^{(\alpha i s}{ }^{\prime)} \mid x_{i}(s) ;, \quad t \leq s
$$

para $i=k+1 . k+2 \ldots . N . \mathrm{E}$, assim, a EDI (3.1), (3.2) tem uma dicotomia exponencial. 
O próximo teorema apresenta condições necessárias para que a EDI (3.1), (3.2) admita uma dicotomia exponencial.

Teorema 6.2 Consideremos a EDI (3.1), (3.2) sobre $\mathbb{R}$. Suponhamos que o operador A seja limitado e que det $Q_{n} \neq 0$, para todo $n \in \mathbb{Z}$. Se (3.1), (3.2) tiver uma dicotomia exponencial entäo, dados $\theta_{1}>1$ e $\theta_{2} \in(0,1)$, existirão una constante $h>0$ e $N$ soluções linearmente independentes da EDI (3.1), (3.2), digamos $x_{1}(t), x_{2}(t), \ldots, x_{N}(t)$, tais que

$$
x_{i}(t) \mid \geq \theta_{\mathrm{l}} \inf \left\{x_{i}(u)|:| u-t \mid \leq h\right\}
$$

$$
\left|x_{i}(t)\right| \leq \theta_{2} \sup \left\{x_{i}(u) \mid: i u-t \leq h\right\}
$$

$i-1,2, \ldots, N$

Demonstração: Suponhamos que a EDI (3.1), (3.2) tenha uma dicotomia exponencial. Pelo Lema 6.5 segue que. dado $\theta_{1}>1$, existem uma constante $h_{1}>0$ e $N$ soluções linearmente independentes da FDI $(3.1),(3.2)$, digamos $x_{1}(t), x_{2}(t), \ldots, x_{N}(t)$, tais que

$$
\left|x_{i}(t)\right| \geq \theta_{1} \inf \left\{! x_{i}(u)|:| u-t \mid \leq h_{1}\right\}
$$

para $i=1,2, \ldots, N$. Como $\theta_{2} \in(0,1)$, pelo Teorema 4.6 , segue que para qualquer solução $x(t)$ da LDI (3.1), (3.2), existe um $h_{2}>0$ tal que

$$
|x(t)| \leq \theta_{2} \sup \left\{|x(u):| u-\cdots t \mid \leq h_{2}\right\}
$$

Assim, para $i=1,2, \ldots N$, existe um $h_{2}^{i}>0$ tal que

$$
x_{i}(t) \mid \leq \theta_{2} \sup \left\{x_{i}(u)|:| u \cdots t \mid \leq h_{2}^{i}\right\}
$$

Tomando $h_{3}=\max \left\{h_{2}^{i}: i=1.2, \ldots . N\right\}$ segue que

$$
\mid x_{i}(t) ! \leq \theta_{2} \sup \left\{\left|x_{i}(u)\right|:|u-t| \leq h_{2}\right\}
$$


Seja $h=\max \left\{h_{1}, h_{3}\right\}$. Assim

$$
\mid x_{i}(t): \geq \theta_{1} \inf \left\{\left|x_{i}(u)\right|:|u-t| \leq h\right\}
$$

$\mathrm{e}$

$$
\left|x_{i}(t)\right| \leq \theta_{2} \sup \left\{\left|x_{i}(u)\right|:|u-t| \leq h\right\} .
$$

Deste modo, o teorema está demonstrado.

O próximo teorema apresenta condições suficientes para que a EDI (3.1), (3.2) admita uma dicotomia exponencial.

Teorema 6.3 Consideremos a EDI (3.1), (3.2) sobre $\mathbb{R}$. Suponhamos que o operador A seja limitado e que det $Q_{n}+0$, para todo $n \in \mathbb{Z}$. Suponhamos, ainda, que existam constantes $\theta_{1}>$ 1, $\theta_{2} \in(0,1)$ e $h>0$ e $N$ soluçôes linearmente independentes da EDI (3.1), (3.2), digamos $x_{1}(t), x_{2}(t), \ldots, x_{N}(t)$, tais que?

$$
\left|x_{i}(t)\right| \geq \theta_{1} \text { inf }\left\{\left|x_{i}(u)\right|:|u-t| \leq h\right\}
$$

$$
x_{i}(t) \mid \leq \theta_{2} \sup \left\{x_{i}(u)|:| u-t \mid \leq h\right\}
$$

$i$ - 1,2,... N. Então a EDI (3.1), (3.2) terá uma dicotomia exponencial.

Demonstração: Como o operador $A$ da EDI (3.1), (3.2) é limitado, para $h>0$ existe uma constante $C \geq 1$ tal que

$$
\left|x_{i}(t)\right| \leq C\left|x_{i}(s)\right|, \quad s \leq t \leq s+h, \quad i \cdots 1,2, \ldots, N
$$

Assim, pelo l.ema 6.4, para quaisquer $i=1,2, \ldots N$ e $t \in \mathbb{R}_{4}$ existem constantes $\alpha_{1}>0$ e $K_{1} \geq 1$ tais que

$$
\left|x_{i}(t): \leq K_{1} e^{\alpha_{1}(t)}\right| x_{i}\left|x_{i}(s)\right|, \quad 0 \leq s \leq t,
$$

ou

$$
\left|x_{i}(t) \leq K_{\mid} e^{\left.-\alpha_{1} ! \cdot s-t\right)}\right| x_{i}(s) \mid, \quad 0 \leq t \leq s .
$$


Novamente, utilizando o I.ema 6.4, para quaisquer $i=1,2, \ldots, N$ e $t \in \mathbb{R}_{-}$, existem constantes $\alpha_{2}>0$ e $K_{2} \geq 1$ tais que

$$
\left|x_{i}(t)\right| \leq K e^{-\alpha(t-s)}\left|x_{i}(s)\right|, \quad s \leq t \leq 0,
$$

ou

$$
\left|x_{i}(t)\right| \leq K e^{-\alpha(s-t)}\left|x_{i}(s)\right|, \quad t \leq s \leq 0 .
$$

Dividiremos a demonstração $\mathrm{cm}$ duas partes: primeiro, suporemos que (6.17) seja verdadeiro e provaremos que isto implicará que apenas (6.19) acontece; depois, suporemos que (6.18) seja verdadeiro e provaremos que isto implicará que apenas (6.20) acontece.

Parte 1: Suponhamos, primeiramente, que (6.17) seja verdadeiro e suponhamos, por absurdo, quc (6.20) aconteça.

De (6.17) e (6.20) segue que

$$
\lim _{i \rightarrow+\infty}\left|x_{i}(t)\right|=0 \quad \text { e } \quad \lim _{t \rightarrow \rightarrow \infty}\left|x_{i}(t)\right|=0
$$

Entāo existe um $\tau_{i} \in \mathbb{R}$ tal que

$$
\left|x_{i}\left(\tau_{i}\right)\right|=\sup \left\{\left|x_{i}(t)\right|: t \in \mathbb{R}\right\} .
$$

Mas isto contradiz a hipótese do teorema. Portanto (6.19) é verdadeiro. Tomando $\alpha=\min \left\{\alpha_{1}, \alpha_{2}\right\}$ e $K=\max \left\{K_{1}, K_{2}\right\}$, de (6.17) e (6.19) obtemos

$$
\left|x_{i}(t)\right| \leq K e^{-\alpha(t \cdot s)} x_{i}(s), \quad s \leq t
$$

Parte 1: Suponhamos, agora, que (6.18) seja verdadeiro e suponhamos, por absurdo, que (6.19) valha.

De (6.18) e (6.19) segue que

$$
\left|x_{i}(s)\right| \geq K_{1}^{\cdots 1} e^{-\alpha_{1}(i-s)} ! x_{i}(t) ; . \quad 0 \leq t \leq s
$$




$$
\left|x_{i}(s)\right| \geq K_{2}^{-1} e^{-\alpha_{2}(s-1)} \mid x_{i}(t) !, \quad s \leq t \leq 0
$$

e estas duas desigualdades implicam

$$
\lim _{s \rightarrow+\infty} \mid x_{i}(s) !=+\infty \quad \text { e } \quad \lim _{s \rightarrow-\infty}\left|x_{i}(s)\right|=+\infty
$$

Então existe um $\bar{\tau}_{i} \in \mathbb{R}$ tal que

$$
\left|x_{i}\left(\bar{\tau}_{i}\right)\right|=\inf \left\{\left|x_{i}(t)\right|: t \in \mathbb{R}\right\} .
$$

Isto contradiz a hipótese do teorema. Portanto (6.20) é verdadeiro. Tomando $\alpha=\min \left\{\alpha_{1}, \alpha_{2}\right\}$ e $K=\max \left\{K_{1}, K_{2}\right\}$, de $(6.18)$ e $(6.20)$ obtemos

$$
\left|x_{i}(t)\right| \leq K e^{-\alpha(s \quad t)}\left|x_{i}(s)\right|, \quad s \geq t
$$

Assim, pelas partes 1 e 2, concluímos que a FDI (3.1), (3.2) tem uma dicotomia exponencial.

No próximo lema, considerando as EDIs (3.1), (3.2) e (3.25), (3.26) sobre o intervalo $J-[a, b]$, através de uma estimativa sobre a diferença de $A(t)$ e $B(t)$, obtemos uma estimativa para os operadores de Cauchy destas EDIs.

Lema 6.7 Consideremos a.s EDIs (3.1), (3.2) e (3.25), (3.26), ambas sobre o intervalo $J=-[a, b]$. Suponhamos que, para cada $t \in[a, b]$, tenhamos

$$
\| B(t)-A(t) !<\varepsilon
$$

então, para $a \leq t, s<b$, teremos

$$
\|V(t . s)-U(t . s)\| \leq L e^{M(b-a) r} e^{J E(b-a)} \cdot 1 !
$$

onde $U(t, s)$ é o operador de evolução da EDI (3.1), (3.2) e V(t,s) é o operador de evolução da EDI (3.25). (3.26). 
Demonstração: Seja

$$
M=\sup \{: \mid A(t) \|: a \leq t \leq b\}
$$

Pelo Lema 3.1, temos

$$
\| U(t, s) \mid \leq L e^{M|t-s|}
$$

para $s, t \in[a, b]$, onde $L=(j-i) \max \left\{\left|Q_{k}\right| i,\left|Q_{k}^{-1}\right| ; ; k=i+1, i+2, \ldots, j\right\}$,

Agora. como $W(t)=V(t, s)-U(t, s)$ é solução da equação não-homogênea

$$
\begin{aligned}
& \frac{d W}{d t}=A(t) W+\left[B(t)-A(t) ;[W(t)+U(t, s)], \quad t \neq t_{n},\right. \\
& W\left(t_{n}^{+}\right)=Q\left(t_{n}\right) W\left(t_{n}\right)+\left[R\left(t_{n}\right)-Q\left(t_{n}\right)\right]\left[W\left(t_{n}\right)+U\left(t_{n}, s\right)\right],
\end{aligned}
$$

com

$$
W(s)=0
$$

Pela Fórmula da Variação das Constantes (Lema 2.1), temos

$$
W(t)=\int_{s}^{t} U(t, \sigma)[B(\sigma)-A(\sigma)][W(\sigma)+U(\sigma, s)] d \sigma,
$$

e, então, se $a \leq s \leq t \leq b$, temos

$$
\begin{aligned}
\|W(t)\| & \leq \int_{s}^{t} L e^{M(t-\sigma)} \mathcal{E}\left[\vdots|W(\sigma)| i+L e^{M(\sigma-s)}\right] d \sigma \\
& \leq L^{2} \varepsilon(t-s) e^{M(t-s)}+L \varepsilon \int_{s}^{t} e^{M(t-\sigma)}\|W(\sigma)\| d \sigma
\end{aligned}
$$

Seja $v(t)=e^{-M t} \mid W(t) \|$. Entāo, para $a \leq s \leq t \leq b$, temos

$$
v(t) \leq \varepsilon L^{2}(t-s) e^{-M s}+L \varepsilon \int_{s}^{t} v(\sigma) d \sigma .
$$

Então, utilizando a Desigualdade de Gronwall Generalizada (Teorema 2.7), segue que

$$
v(t) \leq L^{2} \varepsilon(t-s) e^{-M s}+L^{3} \varepsilon^{2} e^{-k L s} \int_{s}^{t}(\sigma-s) e^{L \varepsilon_{i}(t-\sigma)} d \sigma
$$


e, integrando por partes, chegamos em

$$
v(t) \leq L e^{-M s}\left(e^{\left.L \varepsilon_{(j s)} s\right)}-1\right.
$$

Portanto, para $a \leq s \leq t \leq b$, temos

$$
\|V(t, s)-U(t, s)\| \leq L e^{M(t-s)}\left[e^{L . \varepsilon(t-s)}-1\right]
$$

Podemos provar que, para $a \leq t \leq s \leq b$, temos uma desigualdade similar. Portanto, para $s, t \in|a, b|$

$$
\|V(t, s)-U(t, s)\| \leq L e^{M(b-a)}\left[e^{L \varepsilon(b \cdot a)}-1\right] .
$$

Isto completa a prova do lema.

O teorema a seguir nos diz que, considerando a $\mathrm{EDl}(3.1),(3.2)$ sobre $\mathbb{R}$, se a (3.1), (3.2) admitir uma dicotomia exponencial sobre um intervalo finito de comprimento suficientemente grande, então a EDI (3.1), (3.2) admitirá uma dicotomia exponencial sobre $\mathbb{R}$. Este resultado generaliza o anterior.

Teorema 6.4 Sejam a, $H \in \mathbb{R}, H>0$ e suponhamos que

(i) A EDI (3.1), (3.2) tenha uma dicotomia exponencial sobre o intervalo $[a, a+H]$;

(ii) As funçoes $A(t)$ e $Q(t)$ sejan quase periódicas sobre a reta:

(iii) det $Q_{n} \neq 0$, para qualquer $n \in \mathbb{Z}$

(iv) $\varepsilon$ seja am mímero positivo tal que $L e^{M h}\left(e^{L E h}-1\right) \leq 1 / 2$, onde $M=\sup \|A(s)\|$, $L=(k-i) \max \left\{\left|, Q_{j}\right|,\left\|Q_{j}{ }^{1}\right\| ; j=i, i+1, \ldots, k\right\}$, com $a \in\left(t_{i}, t_{i+1}\right)$ e $a+H \in\left(t_{k}, t_{k+1}\right), e$ $h=\alpha^{\cdots-1}\left(s^{\prime} t^{-1} H+\ln K\right)$.

Se $I I>0$ for tâo grande que $H \geq 4$ e todo internalo de comprimento $H / 2$ contiver um numero E-translação, então a EII (3.1), (3.2) terá uma dicotomia exponencial sobre $\mathbb{R}$. 
Demonstração: Seja $s \in \mathbb{R}$. Então o intervalo $\left[s \cdots \frac{3}{4} H-a, s-\frac{1}{4} H-a\right]$ contém um número $\varepsilon$ translação $\tau$ para $A(t)$. Assim, para todo $t \in\left[s-\frac{3}{4} H-a, s-\frac{1}{4} H \cdots a\right]$,

$$
\|A(t)-A(t-\tau)\|<\varepsilon
$$

Agora, como $\left[s-\frac{3}{4} H-a, s-\frac{1}{4} H-a\right] \subset[a, a-H]$, segue que a EDI

$$
\frac{d y}{d t}=A(t-\tau) y, \quad t-\tau \neq t_{n}
$$

$$
y\left(t_{n}^{+}\right)=Q\left(t_{t}-\tau\right) y\left(t_{n}\right)
$$

tem uma dicotomia exponencial sobre $\left[s-\frac{1}{4} H, s+\frac{1}{4} H\right]$ com constantes $K$ e $\alpha$. Então, como $t \geq 4 h$ e

$$
K^{-1} e^{\alpha h}-K e^{-\alpha h}=2 \operatorname{senh}(\alpha h+\ln K)=2 h
$$

segue do Teorema 6.2, $\operatorname{com} \theta_{1}=\frac{1}{4}$ e $\theta_{2}=4$, que existem $N$ soluções da FDI (6.22), (6.23) linearmente independentes, digamos $y_{i}(t), i=1,2, \ldots, N$, tais que

$$
\left|y_{i}(s)\right| \leq \frac{1}{4} \sup \left\{\left|y_{i}(t)\right|:|t-s| \leq h\right\}
$$

e

$$
\mid y_{i}(s): \geq 4 \inf \left\{\left|y_{i}(t)\right|:|t-s| \leq h\right\}
$$

Agora, seja $x_{i}(t)$ uma solução qualquer de (3.1), (3.2) e seja $y_{i}(t)$ a solução de (6.22), (6.23) $\operatorname{com} y_{i}(s)=x_{i}(s)$. Segue de (6.21) e pela definição de $\varepsilon$ que, para $|t-s| \leq h$, temos

$$
\mid y_{i}(t)-x_{i}(t)_{i}={ }_{1}\left[U(t-\tau, s-\tau)-U(t, s)\left|x_{i}(s) \leq \frac{1}{2}\right| x_{i}(s) \mid\right.
$$

Assim,

$$
\begin{aligned}
\left|x_{i}(s)\right| & =\left|y_{i}(s)\right| \leq \frac{1}{4} \sup \left\{\left|y_{i}(t)\right|: t-s \mid \leq h\right\} \\
& \leq \frac{1}{4} \sup \left\{\left|x_{i}(t)\right|+\left|y_{i}(t) \cdot x_{i}(t):\right| t-s \mid \leq h\right\} \\
& \leq \frac{1}{4} \sup \left\{x_{i}(t)|:| t-s \mid \leq h\right\}+\frac{1}{8} \mid x_{i}(s),
\end{aligned}
$$


ou seja,

$$
\left|x_{i}(s)\right| \leq \frac{2}{7} \sup \left\{\left|x_{i}(t)\right|:|t-s| \leq h\right\}
$$

Também temos

$$
\begin{aligned}
\left|x_{i}(s)\right| & =\left|y_{i}(s)\right| \geq 4 \inf \left\{\left|y_{i}(t)\right|:|t \cdots s| \leq h\right\} \\
& \geq 4 \inf \left\{\left|x_{i}(t)\right|-\left|y_{i}(t)-x_{i}(t)\right|:|t-s| \leq h\right\} \\
& \geq 4 \inf \left\{\mid x_{i}(t)^{\prime}: t-s, \leq h\right\}-2\left|x_{i}(s)\right|,
\end{aligned}
$$

ou seja,

$$
\left|x_{i}(s)\right| \geq \frac{4}{3} \inf \left\{\left|x_{i}(t)\right|: t-s \mid \leq h\right\}
$$

Como $s$ é arbitrário temos que

$$
\left|x_{i}(s)\right|<\frac{2}{7} \sup \left\{x_{i}(t)|:| t-s \mid \leq h\right\}
$$

e

$$
\left|x_{i}(s)\right| \geq \frac{4}{3} \inf \left\{\left|x_{i}(t)\right|:|t-s| \leq h\right\} .
$$

Lintão, pelo Teorema 6.3, segue que a EDI (3.1), (3.2) tem uma dicotomia exponencial sobre $\mathbb{R}$. 


\section{Apêndice 1: Um Teorema do Tipo Áscoli-Arzelá}

Neste apêndice, apresentamos um teorema do tipo $\Lambda$ scoli- $\Lambda$ rzelá para funções contínuas por partes. Este teorema foi relevante para a obtenção do Teorema 5.5 desta dissertação.

Seja $\mathscr{P} \mathscr{C}([0, L], X)$ o espaço de todas as funções de $[0, L]$ com valores $\mathrm{em} X$ que são contínuas em $t \neq t_{n}$, têm descontinuidade de primeira espécie em $t=t_{n}$ e são contínuas à esquerda em $t=t_{n}$, $n=1,2, \ldots, m$, onde $m$ indica o número de descontinuidades no intervalo $[0, L]$.

Para cada $u \in \mathscr{P} \mathscr{C}([0, L], X)$, definimos

$$
\tilde{u}(t)= \begin{cases}u(t), & \text { para } t \neq t_{n}, \\ u\left(t_{n}\right), & \text { para } t=t_{n},\end{cases}
$$

$n=1,2, \ldots, m$. Então, dado $\mathscr{D} \subset \mathscr{P} \mathscr{C}([0, L, X)$, definimos $\tilde{D}:=\{\tilde{u}: u \in \mathscr{D}\}$.

Definimos, também,

$$
\tilde{u}_{0}(t)=u(t), \quad t \in\left[0, t_{1} ;\right.
$$

$$
\tilde{u}_{n}(t)= \begin{cases}u(t), & \text { para } t \neq t_{n}, \\ u\left(t_{n}^{+}\right), & \text {para } t=t_{n},\end{cases}
$$

para $t \in\left[t_{n} \cdot t_{n+1}\right], n=1,2 \ldots \ldots m$. Deste modo, $\tilde{u}_{0} \in \mathscr{C}\left(\left[0 . t_{1}\right], X\right)$ e $\tilde{u}_{n} \in \mathscr{C}\left(\left[t_{n}, t_{n+1}\right], X\right), n=$ $1.2, \ldots m$.

Teorema 6.5 (Teorema de Áscoli-Arzelá para EDIs) [/12], Teorema 3.4J Um conjunto 
$\mathscr{H} \subset \mathscr{B} \mathscr{C}([0, L], X)$ será relativamente compacto se, e somente se, as seguintes propriedades forem verdadeiras:

(i) Para qualquer $t \in \mathbb{R}_{+}$, os conjuntos $\mathscr{D}(t)=\{u(t): u \in \mathscr{D}\}$ e $\tilde{D}(t)=\{\tilde{u}(t): u \in \mathscr{D}\}$ forem relativamente compactos;

(ii) O conjunto $\mathscr{D}(t)$ for equiicontínuo à esquerda em cada $t \in\left[0, L_{\lrcorner}\right.$;

(iii) O conjunto $\tilde{\mathscr{B}}(t)$ for eqüicontínuo à direita em cada $t \in[0, L]$.

Demonstração: Suponhamos que as condições (i), (ii) e (iii) estejam satisfeitas. É consequiência direta das hipóteses e do Teorema de Ascoli-Arzelá clássico que os conjuntos $\tilde{\mathscr{V}}_{0} \mathrm{e} \tilde{\mathscr{V}}_{n}$, são relativamente compactos em $\mathscr{C}\left(\left[0, t_{1}\right], X\right) \mathrm{e}^{\mathscr{C}}\left(\left[t_{n}, t_{n-1}\right], X\right)$ respectivamente, $n=1,2, \ldots, m$.

Agora, provaremos (i) e (iii). A prova de (ii) é análoga à de (iii).

Seja $\left\{u^{k}\right\}_{k \in \mathbb{N}}$ uma sequiência em $\mathscr{D}$. Então existe uma subseqüência de $\left\{u^{k}\right\}_{k \subset \mathbb{N}}$, que denotaremos por $\left\{u^{k_{1}}\right\}_{k_{1} \in \mathbb{N}}$, tal que $\left\{\tilde{u}^{k_{1}}\right\}_{k_{1} \in \mathbb{N}}$ converge para uma função $v_{1} \in \mathscr{C}^{\prime}\left(\left[0, t_{1}\right], X\right)$. Analogamente, a seqüência $\left\{u^{k_{1}}\right\}_{k_{1} \in \mathbb{N}}$ possui uma subsequiencia, que denotaremos por $\left\{u^{k_{2}}\right\}_{k_{2} \in \mathbb{N}}$, tal que $\left\{\tilde{u}^{k_{2}}\right\}_{k_{2} \in \mathbb{N}}$ converge para uma função $v_{2} \in \mathscr{C}\left(\left[t_{1}, t_{2}\right], X\right)$. Continuando com este procedimento, concluímos que existe uma subsequiência de $\left\{u^{k_{n-1}}\right\}_{k_{n-1} \in \mathbb{N}}$, que denotaremos por $\left\{u^{k_{n}}\right\}_{k_{n} \in \mathbb{N}}$, tal que $\left\{\tilde{u}^{k_{n}}\right\}_{k_{n} \in \mathbb{N}}$ converge para uma funçāo $v_{n} \in \mathscr{C}\left(\left[t_{n-1}, t_{n}\right], X\right)$. Então, existe uma subsequiência $\left\{u^{k_{n}}\right\}_{k_{n} \in \mathbb{N}}$ que converge para uma função $u \in \mathscr{P} \mathscr{C}([0, L], X)$, onde $u$ é uma função tal que $\tilde{u}_{n}=v_{n}$, para cada $n=1,2, \ldots, m$. Logo $\mathscr{f}$ é relativamente compacto.

Suponhamos, agora. que $\mathscr{D} \subset \mathscr{C}([0, L], X)$ seja relativamente compacto. Pelo Teorema de Ascoli-Arzelá usual, vemos facilmente que, para $t \neq t_{n}, n=1,2, \ldots, m$, os conjuntos $\mathscr{R}(t)$ e $\tilde{\mathscr{D}}(t)$ são relativamente compactos e que é é equicontínuo em $t$.

Para estudarmos o caso em que $t=t_{n}$, mostraremos, primeiramente, que para cada $n=1,2, \ldots, m$, a função $\psi_{n}(u)=u\left(t_{n}^{+}\right)$é contínua.

Sejam $\varepsilon>0$ e $u \subset \not, G([0, L], X)$. Para todo $v \in B_{\mathcal{\varepsilon} / 3}(u)$, existe $\delta_{v}>0$ tal que

$$
\| v\left(t_{n}^{+}\right)-\left.v\left(t_{n}+h\right)\right|_{i}<\varepsilon / 3
$$


quando $0<h<\delta_{1}$. Com estas escolhas, para $v \in B_{\varepsilon / 3}(u)$ e $0<h<\delta: \min \left\{\delta_{u}, \delta_{v}\right\}$, temos

$$
\left\|u\left(t_{n}^{+}\right)-v\left(t_{n}^{+}\right)\right\| \leq\left\|u\left(t_{n}^{+}\right)-u\left(t_{n}+h\right)\right\|+\left\|u\left(t_{n}+h\right)-v\left(t_{n}+h\right)\right\|+\left\|v\left(t_{n}^{+}\right)-v\left(t_{n}+h\right)\right\|<\varepsilon,
$$

o que prova que $\psi_{n}$ é contínua e, portanto, $\tilde{\mathscr{D}}\left(t_{n}\right)$ é relativamente compacto em $X$.

Mostraremos, agora, a eqüicontinuidade de $\tilde{\mathscr{n}}$ à direita de $t=t_{h}$ raciocinando por absurdo. Suponhamos que existam $\varepsilon>0$ e sequiências $\left\{u^{k}\right\}_{k \in \mathbb{N}}$ em $\mathscr{B} \mathscr{C}([0, L], X)$ e $\left\{h_{i}\right\}_{i \in \mathbb{N}}$ em $\mathbb{R}$, com $0<h_{i}<1 / i$, tais que

$$
\left|u^{k}\left(t_{n}^{+}\right) \cdot u^{k}\left(t_{n}+h_{i}\right)\right| \geq \varepsilon
$$

Como $\mathscr{D}$ é relativamente compacto em $\mathscr{P} \mathscr{C}([0, L], X)$, segue que existem uma subsequiência de $\left\{u^{k}\right\}_{k \in \mathbb{N}}$, que denotaremos por $\left\{u^{k_{1}}\right\}_{k_{1} \in \mathbb{N}}$, e $u \in \mathscr{P} \mathscr{C}([0, L], X)$ tais que $u^{k_{1}} \rightarrow u \mathrm{em} \mathscr{\mathscr { P }} \mathscr{C}^{\prime}([0, L], X)$ quando $k \rightarrow \infty$.

Agora, pela continuidade de $\psi_{n}$, podemos fixar $N_{\varepsilon} \in \mathbb{N}$ tal que

$$
\| u\left(t_{n}^{+}\right)-u^{k}\left(t_{n}^{+}\right) \mid<\varepsilon, \quad n=1,2, \ldots, m
$$

e

$$
\left\|u^{k}-u\right\|<\varepsilon / 3
$$

para todo $k \geq N_{\varepsilon}$. Nestas condições, para $k \geq N_{\varepsilon}$, temos

$$
\begin{aligned}
\left\|u^{k}\left(t_{n}^{+}\right) \quad u^{k}\left(t_{n}+h_{n}\right)\right\| & \geq\left\|u^{k}\left(t_{n}^{+}\right)-u^{k}\left(t_{n}+h\right)\right\|-\left\|u^{k}\left(t_{n}+h\right)-u\left(t_{n}+h\right)\right\|- \\
& -\left\|u\left(t_{n}^{+}\right)-u^{k}\left(t_{n}^{+}\right)\right\|> \\
& >\varepsilon-\varepsilon / 3-\varepsilon / 3=\varepsilon / 3,
\end{aligned}
$$

o que é absurdo, pois $u \in \mathscr{C}([0, L] . X)$. Isto mostra que $\tilde{y}$ é equicontínua à direita em cada $t_{n}$, $n=1,2, \ldots, m$. Portanto a prova do teorema está completa. 


\section{Referências Bibliográficas}

[1] D. D. Bainov, S. I. Kostadinov and Nguyen van Minh, Dichotomies and Integral Manifolds of Impulsive Differential Equations, Science Culture Technology Publishing, Singapore, 1994.

[2] D. D. Bainov, S. I. Kostadinov and P. P. Zabreiko, Stability of the exponential dichotomy of linear impulsive differential equations, Riv. Mat. Univ. Parma, (4) 17, (1991), 271-277.

[3] W. A. Coppel, Dichotomies in Stability Theory, Springer-Verlag, Berlin Heidelberg New York, 1978.

[4] Ju.L. Daleckii and M. G. Krein, Stability of Solutions of Differential Equations in Banach Space, American Mathematical Society Providence, Rhode Island, 1974.

[5] J. K. Hale, Ordinary differential equations, R. E. Krieger Pub. Co., Huntington - New York, 1980.

[6] S. B. Krishna, G. V. Ravindranadh Babu, and J. Vasundhara Devi, Dichotomies for Impulsive Differential Equations, Journal of Mathematical Analysis and Applications 163, (1992), 322344.

17] S. Lang, Analysis II, Addison-Wesley Pub. Co Inc, Reading - Massachuts, 1969.

[8] V. Lakshmikantham, D. D. Bainov and P. S. Simeonov, Theory of Impulsive Differential Equations, World Scientific Publishing Co. Pte. L.td., Singapore-New Jersey-London-Hong Kong, 1989

[9] A. L. Moreno e M. Federson, Dicotomia exponencial e admissibilidade èm equações diferenciais com impulsos, Seminário Brasileiro de Análise - SBA. 60, (2004), 31-38. 
[10] A. L. Moreno e M. Federson, Exponential Dychotomy for Impulsive Differential Equations, Pré-Print.

[11] R. Naulin, A remark on exponential dichotomies, Revista Colombiana de Matematicas. 33, (1999), 9-13.

[12] S. H. Nogueira, Resultados de Existência de Soluçôes para uma Equação Diferencial Funcional com Impulsos, Dissertação de Mestrado, ICMC-USP, São Carlos, 2002. 